\title{
CUSP FORMS FOR REDUCTIVE SYMMETRIC SPACES OF SPLIT RANK ONE
}

\author{
ERIK P. VAN DEN BAN AND JOB J. KUIT
}

\begin{abstract}
For reductive symmetric spaces $G / H$ of split rank one we identify a class of minimal parabolic subgroups for which certain cuspidal integrals of Harish-Chandra-Schwartz functions are absolutely convergent. Using these integrals we introduce a notion of cusp forms and investigate its relation with representations of the discrete series for $G / H$.
\end{abstract}

\section{Contents}

Introduction

1. Notation and preliminaries

2. Radon transforms

3. Harish-Chandra transforms

4. Harish-Chandra-Schwartz functions

5. Fourier transforms

6. The $\tau$-spherical Harish-Chandra transform

7. Extension to the Schwartz space

8. Cusp forms and discrete series representations

Acknowledgments

References

\section{INTRODUCTION}

In this article we aim to develop a notion of cusp forms for reductive symmetric spaces. More precisely, we generalize Harish-Chandra's notion of cusp forms for reductive Lie groups to a notion for reductive symmetric spaces of split rank one. Furthermore, we investigate the relation of this notion with representations of the discrete series for the spaces considered.

Let $G$ be a real reductive Lie group of the Harish-Chandra class and let $\mathscr{C}(G)$ be the Harish-Chandra space of $L^{2}$-Schwartz functions on $G$. In HC75 HarishChandra proved that for every parabolic subgroup $P=M_{P} A_{P} N_{P}$ of $G$, every $\phi \in \mathscr{C}(G)$ and every $g \in G$ of the integral

$$
\int_{N_{P}} \phi(g n) d n
$$

Received by the editors February 25, 2017, and, in revised form, August 28, 2017.

2010 Mathematics Subject Classification. Primary 22E45, 43A85; Secondary 44A12.

The second author was supported by the Danish National Research Foundation through the Centre for Symmetry and Deformation (DNRF92) and the ERC Advanced Investigators Grant HARG 268105. 
is absolutely convergent. In analogy with the theory of automorphic forms, he then defined a cusp form on $G$ to be a function $\phi \in \mathscr{C}(G)$ such that the integral (I.1) vanishes for every proper parabolic subgroup $P$ of $G$ and every $g \in G$. Let $\mathscr{C}_{\text {cusp }}(G)$ be the space of cusp forms and let $\mathscr{C}_{\mathrm{ds}}(G)$ be the closed span of the $K$-finite matrix coefficients of the representations from the discrete series. Harish-Chandra established the fundamental result that

$$
\mathscr{C}_{\text {cusp }}(G)=\mathscr{C}_{\text {ds }}(G)
$$

See [HC66, [HC70, Thm. 10] and [HC75, Sects. 18 \& 27]; see also Var77, Thm. 16.4.17].

For the more general class of real reductive symmetric spaces $G / H$, the main problem one encounters when trying to define cusp forms, is convergence of the integrals involved. The naive idea would be to use the class of $\sigma$-parabolic subgroups, as they appear in the general Plancherel theorem as obtained by P. Delorme Del98 and, independently, H. Schlichtkrull and the first named author, vdBS97c, vdBS05. This approach fails, however, for two reasons: first, the integrals need not always converge (see [AFJS12, Lemma 4.1]) and second, the notion differs from Harish-Chandra's for the group.

Around 2000, M. Flensted-Jensen proposed a notion of cusp forms for symmetric spaces that does generalize Harish-Chandra's notion. This notion makes use of minimal parabolic subgroups for the group $G$, which are in a certain position relative to the Lie algebra $\mathfrak{h}$ of $H$; in a sense they are as far away from $\sigma$-parabolic subgroups as possible; in the present paper such minimal parabolic subgroups are called $\mathfrak{h}$ extreme; see Definition 1.1

In AFJS12 the new notion was tested for real hyperbolic spaces. In that setting the space $\mathscr{C}_{\text {cusp }}(G / H)$ of cusp forms in the Schwartz space $\mathscr{C}(G / H)$ is contained in the discrete part $\mathscr{C}_{\mathrm{ds}}(G / H)$, but in contrast with the case of the group, the inclusion may be proper. The aim of this paper is to understand such and other properties of cusp forms in the more general context of reductive symmetric spaces of split rank one.

Our approach to the convergence problem is indirect, and heavily based on the available tools from the harmonic analysis leading to the Plancherel formula. In an earlier paper, vdBK14, we prepared for the present one by developing (without restriction on the rank) a notion of minimal Eisenstein integrals for $G / H$ in terms of minimal parabolics of the group $G$. For the case of the group viewed as a symmetric space, Harish-Chandra's (minimal) Eisenstein integrals can then be recovered by making the special choice of $\mathfrak{h}$-extreme minimal parabolic subgroups.

Somewhat surprisingly, it appears that for the convergence of the cuspidal integrals another condition on the minimal parabolic subgroup involved is needed, which we call $\mathfrak{h}$-compatibility; see Definition 7.13 . The set $\mathscr{P}_{\mathfrak{h}}$ of such minimal parabolic subgroups is non-empty; for the group, it actually consists of all minimal parabolic subgroups. For the real hyperbolic spaces the class of $\mathfrak{h}$-extreme minimal parabolic subgroups turns out to coincide with the class of $\mathfrak{h}$-compatible ones.

Let $G / H$ be of split rank one. In Theorem 7.23 we prove that for each $Q \in \mathscr{P}_{\mathfrak{h}}$ and every Schwartz function $\phi \in \mathscr{C}(G / H)$ the following Radon transform integral,

$$
\mathcal{R}_{Q} \phi(g):=\int_{N_{Q} / N_{Q} \cap H} \phi(g n) d n \quad(g \in G)
$$


is absolutely convergent and defines a smooth function of $g \in G$; here, $N_{Q}$ denotes the nilpotent radical of $Q$. A function $\phi \in \mathscr{C}(G / H)$ is said to be a cusp form if for all $Q \in \mathscr{P}_{\mathfrak{h}}$ the Radon transform $\mathcal{R}_{Q} \phi$ is identically zero. It turns out that for this to be valid, it is enough to require vanishing of $\mathcal{R}_{Q} \phi$ for all $\mathfrak{h}$-extreme parabolic subgroups in $\mathscr{P}_{\mathfrak{h}}$; see Lemma 8.14. Thus, for both the case of the group and for the real hyperbolic spaces, our notion coincides with the existing ones. Let $\mathscr{C}_{\text {cusp }}(G / H)$ denote the space of cusp forms. Under the assumption of split rank one, we show that

$$
\mathscr{C}_{\text {cusp }}(G / H) \subseteq \mathscr{C}_{\mathrm{ds}}(G / H)
$$

see Theorem 8.20, Let $K$ be a $\sigma$-stable maximal compact subgroup of $G$ and $\tau$ a finite dimensional unitary representation of $K$. In Theorem 8.24 we establish that the space $\mathscr{C}_{\mathrm{ds}}(G / H: \tau)$ admits an $L^{2}$-orthogonal decomposition

$$
\mathscr{C}_{\text {ds }}(G / H: \tau)=\mathscr{C}_{\text {cusp }}(G / H: \tau) \oplus \mathscr{C}_{\text {res }}(G / H: \tau),
$$

where $\mathscr{C}_{\text {res }}(G / H: \tau)$ is spanned by certain residues of Eisenstein integrals defined in terms of $\mathfrak{h}$-compatible, $\mathfrak{h}$-extreme parabolic subgroups. Furthermore, in Theorem 8.22 we give the following remarkable criterion for the analogue of (I.2) to be valid,

$$
\mathscr{C}_{\text {res }}(G / H)^{K}=0 \Rightarrow \mathscr{C}_{\text {cusp }}(G / H)=\mathscr{C}_{\mathrm{ds}}(G / H) .
$$

Finally, we establish, in Theorem 8.26, a characterisation of the subspace $\mathscr{C}_{\mathrm{ds}}(G / H)$ of $\mathscr{C}(G / H)$ in terms of the behavior of the Radon transforms $\mathcal{R}_{Q} \phi$, for $Q \in \mathscr{P}_{\mathfrak{h}}$.

We will now give a more detailed outline of the structure of our paper. In the first part we work in the generality of an arbitrary reductive symmetric space of the Harish-Chandra class. Let $\theta$ be the Cartan involution associated with $K$, and $\mathfrak{g}=\mathfrak{k} \oplus \mathfrak{p}$ the associated Cartan decomposition of the Lie algebra of $G$. Let $\mathfrak{q}$ be the -1-eigenspace of the infinitesimal involution $\sigma$ and let $\mathfrak{a}$ be a maximal abelian subspace of $\mathfrak{p}$ such that $\mathfrak{a}_{\mathfrak{q}}:=\mathfrak{a} \cap \mathfrak{q}$ is maximal abelian in $\mathfrak{p} \cap \mathfrak{q}$. Furthermore, let $A:=\exp \mathfrak{a}$ and $A_{\mathfrak{q}}:=\exp \mathfrak{a}_{\mathfrak{q}}$. The (finite) set of minimal parabolic subgroups $Q \subseteq G$ containing $A$ is denoted by $\mathscr{P}(A)$ and the subset of $\mathfrak{h}$-compatible ones by $\mathscr{P}_{\mathfrak{h}}(A)$. After necessary preparations in Section 1, we define Radon transforms for $\phi \in L^{1}(G / H)$ as in (I.3). By a Fubini type argument combined with the Dixmier-Malliavin theorem on smooth vectors, we show, in Proposition 2.11 that for $\phi \in L^{1}(G / H)^{\infty}$ the integral (I.3) is absolutely convergent, and defines a smooth function on $G / N_{Q}$.

To make the connection with harmonic analysis, we define, in Section 3 a HarishChandra transform $\mathcal{H}_{Q}$, which maps a function $\phi \in L^{1}(G / H)^{\infty}$ to the smooth function on $M A:=Z_{G}(\mathfrak{a})$ given by

$$
\mathcal{H}_{Q} \phi(l)=\delta_{Q}(l) \mathcal{R}_{Q} \phi(l) \quad(l \in M A) .
$$

Here $\delta_{Q}$ is a character on $M A$ that is chosen such that $\mathcal{H}_{Q} \phi$ is right $(M A \cap H)$ invariant and can therefore be viewed as a smooth function on $M A /(M A \cap H)$. We thus obtain a continuous linear map

$$
\mathcal{H}_{Q}: L^{1}(G / H)^{\infty} \longrightarrow C^{\infty}(M A / M A \cap H) .
$$

It is then shown, that associated with $Q$ there exists a certain $P \in \mathscr{P}(A)$ such that $\delta_{P}^{-1} \mathcal{H}_{Q} \varphi$ vanishes at infinity on $M A /(M A \cap H)$ for all $\phi \in L^{1}(G / H)^{\infty}$. It is a consequence of this result that $\mathcal{R}_{Q}$ vanishes on $L^{1}(G / H) \cap L_{\mathrm{ds}}^{2}(G / H)$; see Theorem 3.6 . 
The next goal is to find a condition on the minimal parabolic subgroup $Q$ to ensure that (I.7) extends continuously from $L^{1}(G / H)^{\infty}$ to the larger space $\mathscr{C}(G / H)$.

Our strategy is to first prove, in Section 4 , that every Schwartz function can be dominated by a non-negative $K$-fixed function from $\mathscr{C}(G / H)$; see Proposition 4.2 . Based on this, we show that for the convergence of the integral (I.3) for $\phi \in \mathscr{C}(G / H)$ it suffices to prove that the restriction of $\mathcal{H}_{Q}$ to $C_{c}^{\infty}(G / H)^{K}$ extends continuously to $\mathscr{C}(G / H)^{K}$; see Proposition 4.6.

In Section 5 we use the Eisenstein integrals associated with $Q \in \mathscr{P}(A)$ and a finite dimensional representation $\tau$ of $K$, introduced in vdBK14, to define a Fourier transform $\mathfrak{F}_{Q, \tau}$. For a compactly supported smooth $\tau$-spherical function $\phi \in C_{c}^{\infty}(G / H: \tau)$, the Fourier transform $\mathfrak{F}_{Q, \tau} \phi$ is a meromorphic function of a spectral parameter $\lambda \in \mathfrak{a}_{\mathfrak{q} C}^{*}$.

In Section 6 we introduce a $\tau$-spherical version of the transform (I.7),

$$
\mathcal{H}_{Q, \tau}: C_{c}^{\infty}(G / H: \tau) \rightarrow C^{\infty}\left(A_{\mathfrak{q}}\right) \otimes \mathscr{A}_{M, 2}(\tau) .
$$

Here, $\mathscr{A}_{M, 2}(\tau)$ is a certain finite dimensional Hilbert space, which appears in the description of the most continuous part of the Plancherel formula for $G / H$, as a parameter space for the Eisenstein integrals involved. The transform $\mathcal{H}_{Q, \tau}$ applied to a compactly supported smooth $\tau$-spherical function $\phi \in C_{c}^{\infty}(G / H: \tau)$ gives a function whose Euclidean Fourier-Laplace transform coincides with $\mathfrak{F}_{Q, \tau} \phi$; see Proposition 6.4. At the end of the section, we discuss the relation of the HarishChandra transform with invariant differential operators on $G / H$.

Section 7 is devoted to the extension of the Harish-Chandra transform to the Schwartz space. First, for a function $\phi \in \mathscr{C}(G / H: \tau)$, the transform $\mathcal{H}_{Q, \tau} \phi$ can be expressed as a Euclidean inverse Fourier transform of $\mathfrak{F}_{Q, \tau} \phi$ which involves a contour integral over a translate of $i \mathfrak{a}_{\mathfrak{q}}^{*}$ in the spectral parameter space $\mathfrak{a}_{\mathfrak{q C}}^{*}$; see Lemma 7.1. The idea is then to shift the contour integral towards the tempered part of the Plancherel spectrum, corresponding to $i \mathfrak{a}_{\mathfrak{q}}^{*}$, and to analyze the appearing residues.

At this point we restrict to spaces $G / H$ with $\operatorname{dim} A_{\mathfrak{q}}=1$, in order to be able to handle the appearing residues. The shift then results in the sum of a so-called tempered term and a so-called residual term, which essentially is a sum of residues of the Fourier transform $\mathfrak{F}_{Q, \tau} \phi$. By its close relation with the most continuous part of the Plancherel formula, the tempered term can be shown to extend continuously to the Schwartz space. On the other hand, for $\tau$ the trivial representation, the residual term can be shown to come from testing with matrix coefficients of the discrete series, which arise from residues of the Eisenstein integral for $Q$. It is for drawing this conclusion that the condition of $\mathfrak{h}$-compatibility on $Q$ is needed. Accordingly, for such a $Q$, the transform $\mathcal{H}_{Q, 1}$ extends continuously to all of $\mathscr{C}(G / H)^{K}$. As we indicated above this implies that $\mathcal{R}_{Q}$ extends continuously to $\mathscr{C}(G / H)$; see Theorem 7.23. In turn, this implies that the general $\tau$-spherical Harish-Chandra transform extends to $\mathscr{C}(G / H: \tau)$, so that the associated $\tau$-spherical residual term must be tempered.

In Section 8, 8.1 and 88.2 , we apply a spectral analysis involving invariant differential operators, to show that the $\tau$-spherical residual term consists of matrix coefficients of discrete series representations. In the final subsection of the paper, 8.3. we define the notion of cusp form as discussed above, and obtain the mentioned results (I.4), (I.5) and (I.6) , as well as the mentioned characterization of $\mathscr{C}_{\mathrm{ds}}(G / H)$ in terms of Radon transforms. 
Whenever possible, we develop the theory without restriction on the split rank of $G / H$. In fact, only in the subsections $7.2,7.4,7.5,8.2$ and 8.3 do we require that $\operatorname{dim} A_{\mathfrak{q}}=1$. This restriction will always be mentioned explicitly.

In Remarks 7.24, 8.15, 8.16, 8.21, 8.23 and 8.25] we compare our results with the results of AFJS12. Finally, our results are consistent with the convergence of a certain integral transform appearing in the proof of the Whittaker Plancherel formula given in Wal92, but suggest that the image space does not consist of Schwartz functions. This is confirmed by an explicit calculation for $\operatorname{SL}(2, \mathbb{R})$; see Example 7.4 and Remark 7.5 .

\section{Notation AND PRELiminaries}

Throughout the paper, $G$ will be a reductive Lie group of the Harish-Chandra class, $\sigma$ an involution of $G$ and $H$ an open subgroup of the fixed point subgroup for $\sigma$. We assume that $H$ is essentially connected as defined in vdB86, p. 24]. The involution of the Lie algebra $\mathfrak{g}$ of $G$ obtained by deriving $\sigma$ is denoted by the same symbol. Accordingly, we write $\mathfrak{g}=\mathfrak{h} \oplus \mathfrak{q}$ for the decomposition of $\mathfrak{g}$ into the +1 and -1-eigenspaces for $\sigma$. Thus, $\mathfrak{h}$ is the Lie algebra of $H$. Here and in the rest of the paper, we adopt the convention to denote Lie groups by Roman capitals, and their Lie algebras by the corresponding Fraktur lower cases.

Given a subgroup $S$ of $G$ we agree to write

$$
H_{S}:=S \cap H .
$$

We fix a Cartan involution $\theta$ that commutes with $\sigma$ and write $\mathfrak{g}=\mathfrak{k} \oplus \mathfrak{p}$ for the corresponding decomposition of $\mathfrak{g}$ into the +1 - and -1-eigenspaces for $\theta$. Let $K$ be the fixed point subgroup of $\theta$. Then $K$ is a $\sigma$-stable maximal compact subgroup with Lie algebra $\mathfrak{k}$. In addition, we fix a maximal abelian subspace $\mathfrak{a}_{\mathfrak{q}}$ of $\mathfrak{p} \cap \mathfrak{q}$ and a maximal abelian subspace $\mathfrak{a}$ of $\mathfrak{p}$ containing $\mathfrak{a}_{\mathfrak{q}}$. Then $\mathfrak{a}$ is $\sigma$-stable and

$$
\mathfrak{a}=\mathfrak{a}_{\mathfrak{q}} \oplus \mathfrak{a}_{\mathfrak{h}},
$$

where $\mathfrak{a}_{\mathfrak{h}}=\mathfrak{a} \cap \mathfrak{h}$. This decomposition allows us to identify $\mathfrak{a}_{\mathfrak{q}}^{*}$ and $\mathfrak{a}_{\mathfrak{h}}^{*}$ with the subspaces $(\mathfrak{a} / \mathfrak{h})^{*}$ and $(\mathfrak{a} / \mathfrak{q})^{*}$ of $\mathfrak{a}^{*}$, respectively.

Let $A$ be the connected Lie group with Lie algebra $\mathfrak{a}$. We define $M$ to be the centralizer of $A$ in $K$ and write $L$ for the group $M A$. The set of minimal parabolic subgroups containing $A$ is denoted by $\mathscr{P}(A)$.

In general, if $Q$ is a parabolic subgroup, then its nilpotent radical will be denoted by $N_{Q}$. Furthermore, we agree to write $\bar{Q}=\theta Q$ and $\bar{N}_{Q}=\theta N_{Q}$. Note that if $Q \in \mathscr{P}(A)$, then $L$ is a Levi subgroup of $Q$ and $Q=M A N_{Q}$ is the Langlands decomposition of $Q$.

The root system of $\mathfrak{a}$ in $\mathfrak{g}$ is denoted by $\Sigma=\Sigma(\mathfrak{g}, \mathfrak{a})$. For $Q \in \mathscr{P}(A)$ we put

$$
\Sigma(Q):=\left\{\alpha \in \Sigma: \mathfrak{g}_{\alpha} \subseteq \mathfrak{n}_{Q}\right\} .
$$

Let $Z_{\mathfrak{g}}\left(\mathfrak{a}_{\mathfrak{q}}\right)$ denote the centralizer of $\mathfrak{a}_{\mathfrak{q}}$ in $\mathfrak{g}$. We define the elements $\rho_{Q}$ and $\rho_{Q, \mathfrak{h}}$ of $\mathfrak{a}^{*}$ by

$$
\rho_{Q}(\cdot)=\frac{1}{2} \operatorname{tr}\left(\left.\operatorname{ad}(\cdot)\right|_{\mathfrak{n}_{Q}}\right) \quad \text { and } \quad \rho_{Q, \mathfrak{h}}(\cdot)=\frac{1}{2} \operatorname{tr}\left(\left.\operatorname{ad}(\cdot)\right|_{\mathfrak{n}_{Q} \cap Z_{\mathfrak{g}}\left(\mathfrak{a}_{\mathfrak{q}}\right)}\right) .
$$

Let $m_{\alpha}=\operatorname{dim} \mathfrak{g}_{\alpha}$, for $\alpha \in \Sigma$. Then it follows that

$$
\rho_{Q}=\frac{1}{2} \sum_{\alpha \in \Sigma(Q)} m_{\alpha} \alpha, \quad \text { and } \quad \rho_{Q, \mathfrak{h}}=\frac{1}{2} \sum_{\alpha \in \Sigma(Q) \cap \mathfrak{a}_{\mathfrak{h}}^{*}} m_{\alpha} \alpha .
$$


For an involution $\tau$ of $\mathfrak{g}$ that stabilizes $\mathfrak{a}$ we write

$$
\Sigma(Q, \tau):=\Sigma(Q) \cap \tau \Sigma(Q) .
$$

If $Q \in \mathscr{P}(A)$ then $\Sigma(Q) \cap \mathfrak{a}_{\mathfrak{h}}^{*} \subseteq \Sigma(Q, \sigma)$ and $\Sigma(Q) \cap \mathfrak{a}_{\mathfrak{q}}^{*} \subseteq \Sigma(Q, \sigma \theta)$. Furthermore,

$$
\Sigma(Q)=\Sigma(Q, \sigma \theta) \sqcup \Sigma(Q, \sigma)
$$

see vdBK14, Lemma 2.1]. The following definition is consistent with vdBK14, Def. 1.1].

Definition 1.1. Let $Q \in \mathscr{P}(A)$.

(a) The parabolic subgroup $Q$ is said to be $\mathfrak{q}$-extreme if $\Sigma(Q, \sigma)=\Sigma(Q) \cap \mathfrak{a}_{\mathfrak{h}}^{*}$.

(b) The group $Q$ is said to be $\mathfrak{h}$-extreme if $\Sigma(Q, \sigma \theta)=\Sigma(Q) \cap \mathfrak{a}_{\mathfrak{q}}^{*}$.

We define the partial ordering $\preceq$ on $\mathscr{P}(A)$ by

$$
Q \preceq P \Longleftrightarrow \Sigma(Q, \sigma \theta) \subseteq \Sigma(P, \sigma \theta) \text { and } \Sigma(P, \sigma) \subseteq \Sigma(Q, \sigma) .
$$

The condition $Q \preceq P$ guarantees in particular that $H \cap N_{P} \subseteq H \cap N_{Q}$. The latter implies that we have a natural surjective $H$-map $H /\left(H \cap N_{P}\right) \rightarrow H /\left(H \cap N_{Q}\right)$.

Lemma 1.2. Let $Q \in \mathscr{P}(A)$. Then we have the following equivalences:

(a) $Q$ is q-extreme $\Longleftrightarrow Q$ is $\preceq$-maximal;

(b) $Q$ is $\mathfrak{h}$-extreme $\Longleftrightarrow Q$ is $\preceq$-minimal.

Proof. In both (a) and (b) the implications from left to right are obvious from the definitions. The converse implications follow from vdBK14, Lemma 2.6] and BvdB14, Lemma 2.6].

We denote by $\mathscr{P}_{\sigma}\left(A_{\mathfrak{q}}\right)$ the set of minimal $\theta \sigma$-stable parabolic subgroups containing $A_{\mathfrak{q}}$. If $P_{0} \in \mathscr{P}_{\sigma}\left(A_{\mathfrak{q}}\right)$, then $A \subseteq P_{0}$ and we write

$$
\Sigma\left(P_{0}\right):=\left\{\alpha \in \Sigma: \mathfrak{g}_{\alpha} \subseteq \mathfrak{n}_{P_{0}}\right\} \text { and } \Sigma\left(P_{0}, \mathfrak{a}_{\mathfrak{q}}\right):=\left\{\left.\alpha\right|_{\mathfrak{a}_{\mathfrak{q}}}: \alpha \in \Sigma\left(P_{0}\right)\right\}
$$

Then $P_{0} \mapsto \Sigma\left(P_{0}, \mathfrak{a}_{\mathfrak{q}}\right)$ is a bijection from $\mathscr{P}_{\sigma}\left(A_{\mathfrak{q}}\right)$ onto the collection of positive systems for the root system $\Sigma\left(\mathfrak{g}, \mathfrak{a}_{\mathfrak{q}}\right)$ of $\mathfrak{a}_{\mathfrak{q}}$ in $\mathfrak{g}$.

From vdBK14, Lemma 1.2] we recall that a parabolic subgroup $P \in \mathscr{P}(A)$ is $\mathfrak{q}$-extreme if and only if it is contained in a parabolic subgroup $P_{0} \in \mathscr{P}_{\sigma}\left(A_{\mathfrak{q}}\right)$. Furthermore, in that case we must have

$$
\Sigma\left(P_{0}\right)=\Sigma(P, \sigma \theta),
$$

showing that $P_{0}$ is uniquely determined. In accordance with this observation, we agree to write

$$
\mathscr{P}_{\sigma}(A)=\{P \in \mathscr{P}(A): \quad P \text { is } \mathfrak{q} \text {-extreme }\} .
$$

We note that the assignment $P \mapsto P_{0}$ mentioned above defines a surjective map

$$
\mathscr{P}_{\sigma}(A) \rightarrow \mathscr{P}_{\sigma}\left(A_{\mathfrak{q}}\right) \text {. }
$$

For a given $P_{0} \in \mathscr{P}_{\sigma}\left(A_{\mathfrak{q}}\right)$, the fiber of $P_{0}$ for the map (1.2) consists of the parabolic subgroups $P \in \mathscr{P}_{\sigma}(A)$ with $\Sigma(P)=\Sigma\left(P_{0}\right) \cup\left(\Sigma(P) \cap \mathfrak{a}_{\mathfrak{h}}^{*}\right)$. It is readily seen that the map $P \mapsto \Sigma(P) \cap \mathfrak{a}_{\mathfrak{h}}^{*}$ defines a bijection from this fiber onto the set of positive systems for the root system $\Sigma \cap \mathfrak{a}_{\mathfrak{h}}^{*}$.

Remark 1.3. If $\alpha \in \Sigma \cap \mathfrak{a}_{\mathfrak{h}}^{*}$, then the associated root space $\mathfrak{g}_{\alpha}$ is contained in $\mathfrak{h}$; see vdBK14, Lemma 4.1]. Hence, if $P \in \mathscr{P}_{\sigma}(A)$ and $P_{0}$ the unique group in $\mathscr{P}_{\sigma}\left(A_{\mathfrak{q}}\right)$ containing $P$, then $N_{P} H=N_{P_{0}} H$ and $P H=P_{0} H$. In particular, it follows that $P H$ is an open subset of $G$. 
For $Q \in \mathscr{P}(A)$ we define

$$
\mathscr{P}_{\sigma}(A, Q):=\left\{P \in \mathscr{P}_{\sigma}(A): P \succeq Q\right\} .
$$

It follows from Lemma 1.2(a) that this set is non-empty. The following lemma will be used frequently.

Lemma 1.4. Let $Q \in \mathscr{P}(A)$ and $P_{0} \in \mathscr{P}_{\sigma}\left(A_{\mathfrak{q}}\right)$. Then the following assertions are equivalent.

(a) There exists a $P \in \mathscr{P}_{\sigma}(A)$ such that $Q \preceq P \subseteq P_{0}$.

(b) $\Sigma(Q, \sigma \theta) \subseteq \Sigma\left(P_{0}\right)$.

If (b) is valid, then the group $P$ in (a) is uniquely determined.

Proof. First assume (a). Let $P_{0}$ be the unique parabolic subgroup from $\mathscr{P}_{\sigma}\left(A_{\mathfrak{q}}\right)$ containing $P$. Then $\Sigma(Q, \sigma \theta) \subseteq \Sigma(P, \sigma \theta) \subseteq \Sigma\left(P_{0}\right)$. Hence, (b).

Now, assume (b). By the discussion above there exists a unique q-extreme $P$ with $P \subseteq P_{0}$ and $\Sigma(P) \cap \mathfrak{a}_{\mathfrak{h}}^{*}=\Sigma(Q) \cap \mathfrak{a}_{\mathfrak{h}}^{*}$. For this $P$, we have $\Sigma(Q, \sigma \theta) \subseteq \Sigma\left(P_{0}\right)=$ $\Sigma(P, \sigma \theta)$. Furthermore, $\Sigma(P, \sigma)=\Sigma(P) \cap \mathfrak{a}_{\mathfrak{h}}^{*}=\Sigma(Q) \cap \mathfrak{a}_{\mathfrak{h}}^{*} \subseteq \Sigma(Q, \sigma)$. Hence, $Q \preceq P$ and we infer that (a) is valid.

We fix an $\operatorname{Ad}(G)$-invariant symmetric bilinear form

$$
B: \mathfrak{g} \times \mathfrak{g} \rightarrow \mathbb{R}
$$

such that $B$ is $\theta$ - and $\sigma$-invariant, $B$ agrees with the Killing form on $[\mathfrak{g}, \mathfrak{g}]$ and $-B(\cdot, \theta \cdot)$ is positive definite on $\mathfrak{g}$.

Haar measures on compact Lie groups and invariant measures on compact homogeneous spaces will be normalized such that they are probability measures. If $N$ is a simply connected nilpotent Lie subgroup of $G$ with Lie algebra $\mathfrak{n}$, then we will normalize the Haar measure on $N$ such that its pull-back under the exponential map coincides with the Lebesgue measure on $\mathfrak{n}$ normalized according to the restriction of the inner product $-B(\cdot, \theta(\cdot))$.

\section{RADON TRANSFORMS}

2.1. Decompositions of nilpotent groups. Let $P \in \mathscr{P}(A)$. For a given element $X \in \mathfrak{a}_{\mathfrak{q}}$ we define the Lie subalgebra

$$
\mathfrak{n}_{P, X}:=\bigoplus_{\substack{\alpha \in \Sigma(P) \\ \alpha(X)>0}} \mathfrak{g}_{\alpha}
$$

and denote by $N_{P, X}$ the associated connected Lie subgroup of $G$. The following lemma is proved in [BvdB14, Prop. 2.16].

Lemma 2.1. There exists $X \in \mathfrak{a}_{\mathfrak{q}}$ such that

$$
\begin{cases}\alpha(X) \neq 0 \quad \text { if } \quad \alpha \in \Sigma \backslash \mathfrak{a}_{\mathfrak{h}}^{*}, \\ \alpha(X)>0 \quad \text { if } \quad \alpha \in \Sigma(P, \sigma \theta) .\end{cases}
$$

For any such $X$, the multiplication map

$$
N_{P, X} \times H_{N_{P}} \rightarrow N_{P} \quad\left(n, n_{H}\right) \mapsto n n_{H}
$$

is a diffeomorphism. 
The groups $N_{P}$ and $H_{N_{P}}$ are both unimodular. Hence, there exists an $N_{P^{-}}$ invariant measure on $N_{P} / H_{N_{P}}$. We normalize the measure on $N_{P} / H_{N_{P}}$ such that for every $\psi \in C_{c}\left(N_{P}\right)$,

$$
\int_{N_{P}} \psi(n) d n=\int_{N_{P} / H_{N_{P}}} \int_{H_{N_{P}}} \psi(x n) d n d x .
$$

Lemma 2.1 has the following corollary.

Corollary 2.2. Let $X \in \mathfrak{a}_{\mathfrak{q}}$ be as in Lemma 2.1. Let $\phi \in L^{1}\left(N_{P} / H_{N_{P}}\right)$. Then

$$
\int_{N_{P} / H_{N_{P}}} \phi(x) d x=\int_{N_{P, X}} \phi(n) d n .
$$

Lemma 2.3. Let $P, Q \in \mathscr{P}(A)$ and assume that $X \in \mathfrak{a}_{\mathfrak{q}}$ satisfies the conditions of Lemma 2.1. If $Q \preceq P$, then both $N_{P} \cap \bar{N}_{Q}$ and $N_{Q, X}$ are contained in $N_{P, X}$ and the multiplication map

$$
\left(N_{P} \cap \bar{N}_{Q}\right) \times N_{Q, X} \rightarrow N_{P, X}
$$

is a diffeomorphism.

Proof. Since $\Sigma(P, \sigma) \subseteq \Sigma(Q, \sigma)$, it follows that $\Sigma(P) \cap \Sigma(\bar{Q}) \subseteq \Sigma(P, \sigma \theta)$ and we infer that the first inclusion follows.

Let $\alpha \in \Sigma(Q)$ be such that $\alpha(X)>0$. Assume $-\alpha \in \Sigma(P)$. Then $-\alpha$ is negative on $X$ hence cannot belong to $\Sigma(P, \sigma \theta)$ and must belong to $\Sigma(P, \sigma)$. The latter set is contained in $\Sigma(Q, \sigma)$ hence in $\Sigma(Q)$, contradiction. We conclude that $\alpha \in \Sigma(P)$. This establishes the second inclusion.

From the two established inclusions it follows that $N_{P} \cap \bar{N}_{Q}=N_{P, X} \cap \bar{N}_{Q}$, and $N_{Q, X}=N_{P, X} \cap N_{Q}$ and we see that the above map is a diffeomorphism indeed.

Corollary 2.4. Let $P, Q \in \mathscr{P}(A)$ satisfy $Q \preceq P$ and assume that $\varphi \in C(G / H)$ is integrable over $N_{P} / H_{N_{P}}$. Then for almost all $n \in N_{P} \cap \bar{N}_{Q}$ the function $L_{n^{-1}} \varphi$ is integrable over $N_{Q} / H_{N_{Q}}$ and

$$
\int_{N_{P} / H_{N_{P}}} \varphi(x) d x=\int_{N_{P} \cap \bar{N}_{Q}} \int_{N_{Q} / H_{N_{Q}}} \varphi(n y) d n d y
$$

with absolutely convergent outer integral.

Proof. Let $X \in \mathfrak{a}_{\mathfrak{q}}$ be as in Lemma 2.1. Then the result follows from Corollary 2.2 and Lemma 2.3 combined with Fubini's theorem, in view of the normalization of measures on the nilpotent groups involved; see the end of Section 1 .

2.2. Invariance of integrals. As in the previous section, we assume that $Q \in$ $\mathscr{P}(A)$. Recall that $L=M A$. We define the character $\delta_{Q}$ on $L$ by

$$
\delta_{Q}(l)=\left|\frac{\left.\operatorname{det} \operatorname{Ad}(l)\right|_{\mathfrak{n}_{Q}}}{\left.\operatorname{det} \operatorname{Ad}(l)\right|_{\mathfrak{n}_{Q} \cap Z_{\mathfrak{g}}\left(\mathfrak{a}_{\mathfrak{q}}\right)}}\right|^{\frac{1}{2}} \quad(l \in L) .
$$

Since $M$ is compact, it follows from (1.1) that

$$
\delta_{Q}(m a)=a^{\rho_{Q}-\rho_{Q, \mathfrak{h}}} \quad(m \in M, a \in A) .
$$


Lemma 2.5. Let $\phi$ be a measurable function on $G / H$ such that

$$
\int_{N_{Q} / H_{N_{Q}}}|\phi(n)| d n<\infty .
$$

Then for every $l \in H_{L}$ the function $n \mapsto \phi(l n)$ is absolutely integrable on $N_{Q} / H_{N_{Q}}$ and

$$
\delta_{Q}(l) \int_{N_{Q} / H_{N_{Q}}} \phi(\ln ) d n=\int_{N_{Q} / H_{N_{Q}}} \phi(n) d n .
$$

Proof. Assume that $X \in \mathfrak{a}_{\mathfrak{q}}$ satisfies (2.1) and let $l \in H_{L}$. By applying Lemma 2.1. performing a substitution of variables and applying the same lemma once more, we obtain the following identities of absolutely convergent integrals

$$
\begin{aligned}
\int_{N_{Q} / H_{N_{Q}}} \phi(\ln ) d n & =\int_{N_{Q, X}} \phi\left(\ln ^{\prime}\right) d n^{\prime} \\
& =\int_{N_{Q, X}} \phi\left(\ln ^{\prime} l^{-1}\right) d n^{\prime}=D(l)^{-1} \int_{N_{Q, X}} \phi\left(n^{\prime}\right) d n^{\prime} \\
& =D(l)^{-1} \int_{N_{Q} / H_{N_{Q}}} \phi(n) d n,
\end{aligned}
$$

where $D(l)=|\operatorname{det} \operatorname{Ad}(l)|_{\mathfrak{n}_{Q, X}} \mid$.

Thus, it suffices to show that $D(l)$ equals $\delta_{Q}(l)$ as defined in (2.2). Since $H_{L}=$ $(M \cap H)(A \cap H)$ and $M$ is compact, we see that $D=\delta_{Q}=1$ on $M \cap H$ and it suffices to prove the identity for $l=a \in A \cap H$. Equivalently, in view of (2.3), it suffices to prove the identity of Lemma 2.6 below.

Lemma 2.6. Let $X \in \mathfrak{a}_{\mathfrak{q}}$ be as in (2.1). Then

$$
\left.\left(\rho_{Q}-\rho_{Q, \mathfrak{h}}\right)\right|_{\mathfrak{a}_{\mathfrak{h}}}=\left.\sum_{\substack{\alpha \in \Sigma(Q) \\ \alpha(X)>0}} m_{\alpha} \alpha\right|_{\mathfrak{a}_{\mathfrak{h}}} .
$$

Proof. We write $\Sigma(Q, X)$ for the set of roots $\alpha \in \Sigma(Q)$ with $\alpha(X)>0$. For the purpose of the proof, it will be convenient to use the notation

$$
S(\Phi):=\left.\sum_{\alpha \in A} m_{\alpha} \alpha\right|_{\mathfrak{a}_{\mathfrak{h}}},
$$

for $\Phi \subseteq \Sigma$. The expression on the left-hand side of (2.4) equals $\frac{1}{2} S\left(\Sigma(Q) \backslash \mathfrak{a}_{\mathfrak{h}}^{*}\right)$, whereas the expression on the right-hand side equals $S(\Sigma(Q, X))$. We observe that $\Sigma(Q)$ is the disjoint union of the sets $\Sigma(Q, \sigma)$ and $\Sigma(Q, \sigma \theta)$. Furthermore, $S(\Sigma(Q, \sigma \theta))=0$. Hence,

$$
\left.\left(\rho_{Q}-\rho_{Q, \mathfrak{h}}\right)\right|_{\mathfrak{a}_{\mathfrak{h}}}=\frac{1}{2} S\left(\Sigma(Q, \sigma) \backslash \mathfrak{a}_{\mathfrak{h}}^{*}\right) .
$$

Next, we observe that $\Sigma(Q, \sigma) \backslash \mathfrak{a}_{\mathfrak{h}}^{*}$ is the disjoint union of $\Sigma(Q, X) \cap \Sigma(Q, \sigma)$ and $\sigma(\Sigma(Q, X) \cap \Sigma(Q, \sigma))$ so that

$$
\frac{1}{2} S\left(\Sigma(Q, \sigma) \backslash \mathfrak{a}_{\mathfrak{h}}^{*}\right)=S(\Sigma(Q, \sigma) \cap \Sigma(Q, X)) .
$$

Finally, using that $\Sigma(Q, X) \supseteq \Sigma(Q, \sigma \theta)$ we find

$$
\begin{aligned}
S(\Sigma(Q, \sigma) \cap \Sigma(Q, X)) & =S(\Sigma(Q, \sigma) \cap \Sigma(Q, X))+S(\Sigma(Q, \sigma \theta)) \\
& =S(\Sigma(Q, X))
\end{aligned}
$$

and the lemma follows. 
2.3. Convergence of integrals. As before, we assume that $Q \in \mathscr{P}(A)$. If $P \in$ $\mathscr{P}(A)$ is a q-extreme parabolic subgroup, then $P H$ is an open subset of $G$; see Remark 1.3. Its natural image in $G / H$ will be denoted by $P \cdot H$.

Lemma 2.7. Let $P \in \mathscr{P}_{\sigma}(A, Q)$. Then the $G$-invariant measure on $G / H$ and the $L$-invariant measure on $L / H_{L}$ can be normalized so that for every $\phi \in L^{1}(G / H)$,

$$
\int_{P \cdot H} \phi(x) d x=\int_{N_{P} \cap \bar{N}_{Q}} \int_{L / H_{L}} \frac{\delta_{Q}(l)}{\delta_{P}(l)} \int_{N_{Q} / H_{N_{Q}}} \phi(\bar{n} l n) d n d l d \bar{n}
$$

with absolutely convergent integrals.

Note that by Lemma 2.5 the function

$$
L \ni l \mapsto \delta_{Q}(l) \int_{N_{Q} / H_{N_{Q}}} \phi(\ln ) d n
$$

is right $H_{L}$-invariant if the integral is absolutely convergent for every $l \in L$. Since $\delta_{P}$ is a right $H_{L}$-invariant function as well, the right-hand side of (2.5) is well-defined.

Proof of Lemma 2.7. It suffices to prove the lemma for non-negative integrable functions only. Let $\phi \in L^{1}(G / H)$ be non-negative. Since $P \cdot H$ is an open subset of $G / H$, the integral over $P \cdot H$ is absolutely convergent. The repeated integral on the right-hand side of (2.5) is well-defined (although possibly infinitely large). To prove the lemma, we start by rewriting the right-hand side and then show that it equals the left-hand side.

Note that $L / H_{L}$ is diffeomorphic to $M / H_{M} \times A_{\mathfrak{q}}$ and the $L$-invariant measure on $L / H_{L}$ equals the product of the $M$-invariant measure on $M / H_{M}$ and the Haar measure on $A_{\mathfrak{q}}$. Furthermore, from (2.3) we infer that

$$
\frac{\delta_{Q}(m a)}{\delta_{P}(m a)}=a^{\rho_{Q}-\rho_{P}} \quad\left(m \in M, a \in A_{\mathfrak{q}}\right) .
$$

Hence

$$
\begin{aligned}
& \int_{N_{P} \cap \bar{N}_{Q}} \int_{L / H_{L}} \frac{\delta_{Q}(l)}{\delta_{P}(l)} \int_{N_{Q} / H_{N_{Q}}} \phi(\bar{n} l n) d n d l d \bar{n} \\
& =\int_{N_{P} \cap \bar{N}_{Q}} \int_{M} \int_{A_{\mathfrak{q}}} a^{\rho_{Q}-\rho_{P}} \int_{N_{Q} / H_{N_{Q}}} \phi(\bar{n} m a n) d n d a d m d \bar{n}
\end{aligned}
$$

Here we have used that $H_{M}$ is compact and has volume equal to 1 by our chosen normalization of the Haar measure.

Let $P_{0}$ be the unique minimal $\sigma \theta$-stable parabolic subgroup such that $P \subseteq P_{0}$. Then the set of roots of $\mathfrak{a}$ in $\mathfrak{n}_{P_{0}}$ is given by $\Sigma\left(P_{0}\right)=\Sigma(P) \backslash \mathfrak{a}_{\mathfrak{h}}^{*}$ and

$$
P_{0}=Z\left(\mathfrak{a}_{\mathfrak{q}}\right) N_{P_{0}} .
$$

It follows that $\rho_{P}-\rho_{P_{0}}$ vanishes on $\mathfrak{a}_{\mathfrak{q}}$.

Let $X \in \mathfrak{a}_{\mathfrak{q}}$ be such that $\alpha(X)>0$ for every $\alpha \in \Sigma\left(P_{0}\right)$. Then $X$ satisfies (2.1) and it is readily seen that $N_{Q, X}=N_{Q} \cap N_{P_{0}}$. By Corollary 2.2 the integral over $N_{Q} / H_{N_{Q}}$ can be replaced by an integral over $N_{Q} \cap N_{P_{0}}$. Therefore, (2.6) can be rewritten as

$$
\int_{\bar{N}_{Q} \cap N_{P}} \int_{M} \int_{A_{\mathfrak{q}}} a^{\rho_{Q}-\rho_{P_{0}}} \int_{N_{Q} \cap N_{P_{0}}} \phi(\bar{n} m a n) d n d a d m d \bar{n} .
$$


Note that $\left(\bar{N}_{Q} \cap N_{P}\right)=\left(\bar{N}_{Q} \cap N_{P_{0}}\right)$. The multiplication map

$$
\left(\bar{N}_{Q} \cap N_{P_{0}}\right) \times\left(N_{Q} \cap N_{P_{0}}\right) \rightarrow N_{P_{0}}
$$

is a diffeomorphism with Jacobian equal to 1 . We now change the order of integration in (2.7) and subsequently apply the change of variables $n \mapsto(m a) n(m a)^{-1}$ to the integral over $\bar{N}_{Q} \cap N_{P_{0}}$. This change of variables has Jacobian equal to $a^{\rho_{P_{0}}}-\rho_{Q}$ by the lemma below. Finally, we rewrite the double integral over $\bar{N}_{Q} \cap N_{P_{0}}$ and $N_{Q} \cap N_{P_{0}}$ as an integral over $N_{P_{0}}$. We thus infer that the integral in (2.7) equals

$$
\int_{M} \int_{A_{\mathfrak{q}}} \int_{N_{P_{0}}} \phi(\operatorname{man}) d n d a d m
$$

Note that $M_{P_{0}} \cap K \cap H$ centralizes $A_{\mathfrak{q}}$ and normalizes $N_{P_{0}}$. Moreover,

$$
|\operatorname{det} \operatorname{Ad}(m)|_{\mathfrak{n}_{P_{0}}} \mid=1
$$

for all $m \in M_{P_{0}} \cap K \cap H$. Since the volume of $M_{P_{0}} \cap K \cap H$ equals 1 (by our chosen normalization of Haar measure), it follows that the integral (2.8) equals

$$
\int_{M} \int_{M_{P_{0}} \cap K \cap H} \int_{A_{\mathfrak{q}}} \int_{N_{P_{0}}} \phi\left(m m^{\prime} a n\right) d n d a d m^{\prime} d m .
$$

It follows from vdBK14, Lemma 4.3] that $M\left(M_{P_{0}} \cap H\right)=M_{P_{0}}$. Therefore the integrals over $M$ and $M_{P_{0}} \cap K \cap H$ can be replaced by one integral over $M_{P_{0}} \cap K$. To conclude the proof, we note that

$$
\int_{M_{P_{0}} \cap K} \int_{A_{\mathfrak{q}}} \int_{N_{P_{0}}} \phi\left(m m^{\prime} a n\right) d n d a d m=c \int_{P_{0} \cdot H} \phi(x) d x
$$

for some constant $c>0$ by [Óla87, Thm. 1.2], and observe that $P_{0} \cdot H=P \cdot H$; see Remark 1.3 .

Lemma 2.8. Let $P_{0} \in \mathscr{P}_{\sigma}\left(A_{\mathfrak{q}}\right)$ satisfy $\Sigma(Q, \sigma \theta) \subseteq \Sigma\left(P_{0}\right)$. Then

$$
|\operatorname{det} \operatorname{Ad}(m a)|_{\overline{\mathfrak{n}}_{Q} \cap \mathfrak{n}_{P_{0}}} \mid=a^{\rho_{P_{0}}-\rho_{Q}}, \quad\left(m \in M, a \in A_{\mathfrak{q}}\right) .
$$

Proof. Given a subset $\Phi \subseteq \Sigma$ we agree to write

$$
T(\Phi)=\left.\sum_{\alpha \in \Phi} m_{\alpha} \alpha\right|_{\mathfrak{a}_{\mathfrak{q}}} .
$$

Then, the expression on the left-hand side of (2.9) equals

$$
a^{T\left(\theta \Sigma(Q) \cap \Sigma\left(P_{0}\right)\right)} .
$$

Since $\Sigma(Q)$ is the disjoint union of $\Sigma(Q, \sigma)$ and $\Sigma(Q, \sigma \theta)$, whereas the latter set is contained in $\Sigma\left(P_{0}\right)$, it follows that the expression on the left-hand side of (2.9) equals

$$
a^{T\left(\theta \Sigma(Q, \sigma) \cap \Sigma\left(P_{0}\right)\right)} .
$$

On the other hand, the expression on the right-hand side of (2.9) equals

$$
a^{\frac{1}{2}\left[T\left(\Sigma\left(P_{0}\right)\right)-T(\Sigma(Q)]\right.}
$$


Now $T(\Sigma(Q, \sigma))=0$ and since $\Sigma(Q)$ is the disjoint union of $\Sigma(Q, \sigma)$ and $\Sigma(Q, \sigma \theta)$, whereas the latter set is contained in $\Sigma\left(P_{0}\right)$, it follows that

$$
\begin{aligned}
T\left(\Sigma\left(P_{0}\right)\right)-T(\Sigma(Q)) & =T\left(\Sigma\left(P_{0}\right) \backslash \Sigma(Q, \sigma \theta)\right) \\
& =T\left(\Sigma\left(P_{0}\right) \cap \Sigma(Q, \sigma)\right)+T\left(\Sigma\left(P_{0}\right) \cap \theta \Sigma(Q, \sigma)\right) \\
& =T\left(\theta \sigma \Sigma\left(P_{0}\right) \cap \sigma \Sigma(Q, \sigma)\right)+T\left(\Sigma\left(P_{0}\right) \cap \theta \Sigma(Q, \sigma)\right) \\
& =2 T\left(\Sigma\left(P_{0}\right) \cap \theta \Sigma(Q, \sigma)\right) .
\end{aligned}
$$

Combining these we find that the expression on the right-hand side of (2.9) equals (2.10) as well.

Lemma 2.9. Let $P \in \mathscr{P}_{\sigma}(A, Q)$. There exists a constant $c>0$ such that for every $\phi \in L^{1}(G / H)$,

$$
\int_{K} \int_{L / H_{L}} \frac{\delta_{Q}(l)}{\delta_{P}(l)} \int_{N_{Q} / H_{N_{Q}}}|\phi(k \ln )| d n d l d k \leq c\|\phi\|_{L^{1}}
$$

Proof. Applying Lemma 2.7 to left $K$-translates of $\phi$ we find

$$
\begin{aligned}
\|\phi\|_{L^{1}} & =\int_{K} \int_{G / H}|\phi(k \cdot x)| d x d k \geq \int_{K} \int_{P \cdot H}|\phi(k \cdot x)| d x d k \\
& =\int_{K} \int_{\bar{N}_{Q} \cap N_{P}} \int_{L / H_{L}} \int_{N_{Q} / H_{N_{Q}}} \frac{\delta_{Q}(l)}{\delta_{P}(l)}|\phi(k \bar{n} l n)| d n d l d \bar{n} d k .
\end{aligned}
$$

For $g \in G$ we write $k_{Q}(g), a_{Q}(g)$ and $n_{Q}(g)$ for the elements of $K, A$ and $N_{Q}$, respectively, such that the Iwasawa decomposition of $g$ is given by $g=k_{Q}(g) a_{Q}(g) n_{Q}(g)$. Let $C$ be a compact subset of $\theta N_{Q} \cap N_{P}$ with open interior. By a change of the integration variables from $N_{Q} / H_{N_{Q}}, L / H_{L}$ and $K$ we obtain

$$
\begin{aligned}
\|\phi\|_{L^{1}} & \geq \int_{K} \int_{C} \int_{L / H_{L}} \int_{N_{Q} / H_{N_{Q}}} \frac{\delta_{Q}(l)}{\delta_{P}(l)}\left|\phi\left(k k_{Q}(\bar{n}) a_{Q}(\bar{n}) n_{Q}(\bar{n}) \ln \right)\right| d n d l d \bar{n} d k \\
& =\int_{C} \frac{\delta_{P}\left(a_{Q}(\bar{n})\right)}{\delta_{Q}\left(a_{Q}(\bar{n})\right)} d \bar{n} \int_{K} \int_{L / H_{L}} \int_{N_{Q} / H_{N_{Q}}} \frac{\delta_{Q}(l)}{\delta_{P}(l)}|\phi(k l n)| d n d l d k .
\end{aligned}
$$

Note that the first integral on the right-hand side is finite since $C$ is compact. Moreover, it is strictly positive since $C$ has an open interior and the integrand is strictly positive. This proves the lemma.

We denote by $L_{\text {loc }}^{1}\left(G / N_{Q}\right)$ the space of locally integrable functions on $G / N_{Q}$. Let $d x$ be a choice of invariant measure on $G / N_{Q}$. Then for each compact subset $C \subseteq G / N_{Q}$ the function

$$
\nu_{C}: \phi \mapsto \int_{C}|\phi(x)| d x
$$

defines a continuous seminorm on $L_{\text {loc }}^{1}(G / H)$. The seminorms thus defined determine a Fréchet topology on $L_{\text {loc }}^{1}(G / H)$. It is readily seen that the left regular representation of $G$ in $L_{\text {loc }}^{1}(G / H)$ is continuous for this topology.

Corollary 2.10. Let $Q \in \mathscr{P}(A)$. Then for every $\phi \in L^{1}(G / H)$ the integral

$$
{ }^{'} \mathcal{R}_{Q} \phi(g):=\int_{N_{Q} / H_{N_{Q}}} \phi(g n) d n
$$


converges for $g$ in a right $N_{Q}$-invariant measurable subset of $G$ whose complement is of measure zero. The defined function ' $\mathcal{R}_{Q} \phi(g)$ is locally integrable on $G / N_{Q}$. Finally, the resulting map

$$
{ }^{\prime} \mathcal{R}_{Q}: L^{1}(G / H) \rightarrow L_{\text {loc }}^{1}\left(G / N_{Q}\right)
$$

is continuous linear and $G$-equivariant.

Proof. Let $\phi \in L^{1}(G / H)$. By the Iwasawa decomposition, $K A$ is a closed submanifold of $G$. Since the multiplication map $K \times L \rightarrow K A$ defines a fiber bundle with fiber $M$, it follows from Lemma 2.9 combined with Fubini's theorem that there exists a subset $\Omega \subseteq K A$, whose complement has Lebesgue measure zero, such that the integral (2.11) converges for all $x \in \Omega$. Furthermore, the resulting function ${ }^{\prime} \mathcal{R}_{Q} \phi$ is locally integrable on $K A$. By invariance of the measure on $N_{Q} / H_{N_{Q}}$ it follows that (2.11) converges for $g \in \Omega N_{Q}$. By the Iwasawa decomposition the set $\Omega N_{Q}$ has a complement of measure 0 . We infer that the resulting function ${ }^{\prime} \mathcal{R}_{Q} \phi$ is defined almost everywhere and locally integrable on $G / N_{Q}$. By application of Fubini's theorem, it follows from the estimate in Lemma 2.9 that the map ${ }^{\prime} \mathcal{R}_{Q}: L^{1}(G / H) \rightarrow L_{\text {loc }}^{1}\left(G / N_{Q}\right)$ thus defined is continuous linear. Its $G$-equivariance is obvious from the definition.

We write $L^{1}(G / H)^{\infty}$ for the space of smooth vectors for the left-regular representation $L$ of $G$ in $L^{1}(G / H)$. If $\phi \in C^{\infty}(G / H)$ and $L_{u} \phi \in L^{1}(G)$ for all $u \in U(\mathfrak{g})$, then $\phi \in L^{1}(G)^{\infty}$; this follows by a straightforward application of Taylor's theorem with remainder term; see also [Pou72, Thm. 5.1].

Conversely, any function in $L^{1}(G / H)^{\infty}$ can be represented by a smooth function $\phi \in C^{\infty}(G)$. This follows from the analogous local statement in $\mathbb{R}^{n}$ by using a partition of unity. We may thus identify $L^{1}(G / H)^{\infty}$ with the space of $\phi \in C^{\infty}(G / H)$ such that $L_{u} \phi \in L^{1}(G / H)$, for all $u \in U(\mathfrak{g})$.

Likewise, we write $L_{\text {loc }}^{1}\left(G / N_{Q}\right)^{\infty}$ for the Fréchet space of smooth vectors in the $G$-space $L_{\text {loc }}^{1}\left(G / N_{Q}\right)$. By similar remarks as those made above it follows that the inclusion map $C^{\infty}\left(G / N_{Q}\right) \rightarrow L_{\text {loc }}^{1}\left(G / N_{Q}\right)$ induces a topological linear isomorphism

$$
C^{\infty}\left(G / N_{Q}\right) \stackrel{\simeq}{\longrightarrow} L_{\text {loc }}^{1}\left(G / N_{Q}\right)^{\infty} .
$$

By equivariance, it follows from Corollary 2.10 that the map ' $\mathcal{R}_{Q}$ restricts to a continuous linear map $L^{1}(G / H)^{\infty} \rightarrow L_{\text {loc }}^{1}\left(G / N_{Q}\right)^{\infty}$. The following proposition asserts that the integral transform ' $\mathcal{R}_{Q}$ actually sends the smooth representatives for functions in the first of these spaces to smooth representatives of functions in the second.

Proposition 2.11. Let $Q \in \mathscr{P}(A)$ and $\phi \in L^{1}(G / H)^{\infty}$. Then for every $g \in G$ the integral

$$
\mathcal{R}_{Q} \phi(g):=\int_{N_{Q} / H_{N_{Q}}} \phi(g n) d n
$$

is absolutely convergent and the displayed integral defines a smooth function of $g \in G$. The indicated transform defines a continuous linear $G$-equivariant map

$$
\mathcal{R}_{Q}: L^{1}(G / H)^{\infty} \rightarrow C^{\infty}\left(G / N_{Q}\right)
$$

Proof. By [DM78, Thm. 3.3] the space $L^{1}(G / H)^{\infty}$ is spanned by functions of the form $\phi=\chi * \psi$ with $\chi \in C_{c}^{\infty}(G)$ and $\psi \in L^{1}(G / H)$. Therefore, it suffices to prove 
the proposition for such functions. Let $\chi \in C_{c}^{\infty}(G)$ and $\psi \in L^{1}(G / H)$, and put $\phi=\chi * \psi$.

It follows from Corollary 2.10 that the integral for $\chi * \mathcal{R}_{Q} \psi(g)$, given by

$$
J(g):=\int_{G} \chi(g \gamma) \int_{N_{Q} / H_{N_{Q}}} \psi\left(\gamma^{-1} n\right) d n d \gamma
$$

is absolutely convergent for every $g \in G$ and the defined function $J: G \rightarrow \mathbb{C}$ is smooth. By a change of variables, followed by application of Fubini's theorem the integral may be rewritten as

$$
\begin{aligned}
J(g) & =\int_{G} \chi(\gamma) \int_{N_{Q} / H_{N_{Q}}} \psi\left(\gamma^{-1} g n\right) d n d \gamma \\
& =\int_{N_{Q} / H_{N_{Q}}}(\chi * \psi)(g n) d n \\
& =\int_{N_{Q} / H_{N_{Q}}} \varphi(g n) d n .
\end{aligned}
$$

All assertions but the last now follow. By equivariance, it follows that the map ${ }^{\prime} \mathcal{R}_{Q}$ defined in Corollary 2.10 restricts to a continuous linear map $L^{1}(G / H)^{\infty} \rightarrow$ $L_{\text {loc }}^{1}\left(G / N_{Q}\right)^{\infty}$. For $\phi \in L^{1}(G / H)^{\infty}$, the function ' $\mathcal{R}_{Q}(\phi)$ is represented by the smooth function $\mathcal{R}_{Q}(\phi)$. The last assertion now follows from the fact that (2.12) is a topological linear isomorphism.

Definition 2.12. The Radon transform $\mathcal{R}_{Q}$ is defined to be the $G$-equivariant continuous linear map

$$
\mathcal{R}_{Q}: L^{1}(G / H)^{\infty} \rightarrow C^{\infty}\left(G / N_{Q}\right)
$$

given by (2.13).

\section{HARISH-ChANDRA TRANSFORMS}

We retain the assumption that $Q \in \mathscr{P}(A)$. In terms of the Radon transform $\mathcal{R}_{Q}$, defined in the previous section, we define a new transform as follows.

Definition 3.1. The Harish-Chandra transform $\mathcal{H}_{Q}$ is defined to be the continuous linear map

$$
L^{1}(G / H)^{\infty} \rightarrow C^{\infty}\left(L / H_{L}\right)
$$

given by

$$
\mathcal{H}_{Q} \phi(l)=\delta_{Q}(l) \mathcal{R}_{Q} \phi(l) \quad(l \in L) .
$$

Example 3.2 (Group case). Let ' $G$ be a reductive Lie group of the HarishChandra class. Then ' $G$ is diffeomorphic to $G / H$, where $G={ }^{\prime} G \times{ }^{\prime} G$ and $H=$ $\operatorname{diag}(' G)$, via the map

$$
G / H \rightarrow{ }^{\prime} G ; \quad\left(g_{1}, g_{2}\right) \mapsto g_{1} g_{2}^{-1} .
$$

Under this map, the action of $G$ on $G / H$ corresponds to the left times right action of ' $G \times{ }^{\prime} G$ on ' $G$. As $H$ is the fixed-point group of the involution $\sigma: G \rightarrow G$, $\left({ }^{\prime} x,{ }^{\prime} y\right) \mapsto\left({ }^{\prime} y,{ }^{\prime} x\right)$, the pair $(G, H)$ is symmetric. Let $\mathfrak{g}=\mathfrak{k} \oplus \mathfrak{p}$ be a $\sigma$-stable Cartan decomposition of $\mathfrak{g}$. Then $\mathfrak{k}=^{\prime} \mathfrak{k} \times{ }^{\mathfrak{k}}$ and $\mathfrak{p}={ }^{\prime} \mathfrak{p} \times{ }^{\prime} \mathfrak{p}$, where ${ }^{\prime} \mathfrak{g}={ }^{\prime} \mathfrak{k} \oplus{ }^{\prime} \mathfrak{p}$ is a Cartan 
decomposition of ' $\mathfrak{g}$. Let ' $\mathfrak{a}$ be a maximal abelian subspace of ' $\mathfrak{p}$ and let $\mathfrak{a}={ }^{\prime} \mathfrak{a} \times{ }^{\prime} \mathfrak{a}$. Then

$$
\mathfrak{a}_{\mathfrak{q}}:=\mathfrak{a} \cap \mathfrak{q}=\{(H,-H): H \in \mathfrak{\mathfrak { a }}\}
$$

is a maximal abelian subspace of $\mathfrak{p} \cap \mathfrak{q}$. Every minimal parabolic subgroup of $G$ is of the form ' $P \times{ }^{\prime} Q$, where ' $P$ and ' $Q$ are minimal parabolic subgroups of ' $G$. Let ${ }^{\prime} A=\exp \left({ }^{\prime} \mathfrak{a}\right)$ and let $A={ }^{\prime} A \times{ }^{\prime} A$. Let ' $L=Z_{G}\left({ }^{\prime} A\right)$ and let $L={ }^{\prime} L \times{ }^{\prime} L$.

Every $\mathfrak{h}$-extreme parabolic subgroup is of the form ' $P \times^{\prime} P$, where ' $P$ is a minimal parabolic subgroup of ' $G$. Let ' $P$ be a minimal parabolic subgroup containing ' $A$. Under the identifications $G / H \simeq{ }^{\prime} G$ and $L / H_{L} \simeq{ }^{\prime} L$ the transform $\mathcal{H}_{{ }_{P}}:=\mathcal{H}_{{ } P \times P}$ is given by

$$
\mathcal{H}_{\backslash_{P}} \phi(m a)=a^{\rho_{\backslash_{P}}} \int_{N_{\backslash_{P}}} \phi(\operatorname{man}) d n \quad\left(\phi \in L^{1}\left({ }^{\prime} G\right)^{\infty}, m \in{ }^{\prime} M, a \in{ }^{\prime} A\right) .
$$

This shows that $\mathcal{H}_{P}$ equals the map $\phi \mapsto \phi^{\left({ }^{(P)}\right)}$, defined by Harish-Chandra in HC75, p. 145].

Similarly, under the described identifications the Radon transform $\mathcal{R}_{{ }_{P} P}:=\mathcal{R}_{{ }_{P} \times^{\prime} P}$ is given by

$$
\mathcal{R}_{\backslash_{P}} \phi\left(g_{1}, g_{2}\right)=\int_{N_{\backslash_{P}}} \phi\left(g_{1} n g_{2}^{-1}\right) d n \quad\left(\phi \in L^{1}\left({ }^{\prime} G\right)^{\infty}, g_{1}, g_{2} \in G\right) .
$$

The function $\mathcal{R}_{{ }_{P}} \phi(\cdot, e)$ is equal to $\phi^{\prime} P$, defined by Harish-Chandra in $\mathrm{HC75}$, p. 145].

In the remainder of this section we investigate some of the properties of the Harish-Chandra transform. We start with a proposition.

Proposition 3.3. Let $P \in \mathscr{P}_{\sigma}(A, Q)$ and $\phi \in L^{1}(G / H)^{\infty}$. Then

$$
l \mapsto \delta_{P}(l)^{-1} \mathcal{H}_{Q} \phi(l), \quad L / H_{L} \rightarrow \mathbb{C}
$$

defines a function in $L^{1}\left(L / H_{L}\right)^{\infty}$.

Remark 3.4. In particular, the function (3.1) vanishes at infinity by Lemma 3.5 below.

Proof. We will first prove that $\delta_{P}^{-1} \mathcal{H}_{Q} \phi$ is integrable. From [DM78, Thm. 3.3] it follows that $L^{1}(G / H)^{\infty}$ is spanned by functions of the form $\phi=\chi * \psi$ with $\chi \in C_{c}^{\infty}(G)$ and $\psi \in L^{1}(G / H)$. Hence, we may assume that $\phi$ is of this form. It follows from Lemma 2.9 and Fubini's theorem that the integral

$$
\int_{L / H_{L}} \frac{\delta_{Q}(l)}{\delta_{P}(l)} \int_{N_{Q} / H_{N_{Q}}} \psi\left(\gamma^{-1} l n\right) d n d l
$$

is absolutely convergent for almost every $\gamma \in G$, and that the almost everywhere defined function on $G$ thus obtained is locally integrable. Therefore, the integral

$$
\int_{G} \chi(\gamma) \int_{L / H_{L}} \frac{\delta_{Q}(l)}{\delta_{P}(l)} \int_{N_{Q} / H_{N_{Q}}} \psi\left(\gamma^{-1} \ln \right) d n d l d \gamma
$$

is absolutely convergent, and by Fubini's theorem it is equal to

$$
\int_{L / H_{L}} \frac{\delta_{Q}(l)}{\delta_{P}(l)} \int_{N_{Q} / H_{N_{Q}}}(\chi * \psi)(\ln ) d n d l=\int_{L / H_{L}} \frac{\mathcal{H}_{Q}(\phi)(l)}{\delta_{P}(l)} d l .
$$

This proves the integrability of $\delta_{P}^{-1} \mathcal{H}_{Q} \phi$. 
We move on to show that $\delta_{P}^{-1} \mathcal{H}_{Q} \phi \in L^{1}\left(L / H_{L}\right)^{\infty}$. In view of the remarks above (2.12) it suffices to prove that $u\left(\delta_{P}^{-1} \mathcal{H}_{Q} \phi\right)$ is integrable for each $u \in U(\mathfrak{l})$.

For conciseness we write $h:=\delta_{Q}^{-1} \mathcal{H}_{Q} \phi=\left.\mathcal{R}_{Q}(\phi)\right|_{L}$. Let $u \in U(\mathfrak{l})$. By the Leibniz rule there exists an $n \in \mathbb{N}$ and $u_{j}, v_{j} \in U(\mathfrak{l})$ for $1 \leq j \leq n$, such that

$$
u\left(\delta_{P}^{-1} \mathcal{H}_{Q} \phi\right)=u\left(\frac{\delta_{Q}}{\delta_{P}} h\right)=\sum_{j=1}^{n}\left(u_{j} \frac{\delta_{Q}}{\delta_{P}}\right) v_{j}(h) .
$$

Since $\delta_{Q} / \delta_{P}$ is a character on $L$, there exist constants $c_{j}$ such that $u_{j}\left(\delta_{Q} / \delta_{P}\right)=$ $c_{j} \delta_{Q} / \delta_{P}$. Therefore,

$$
\begin{aligned}
u\left(\delta_{P}^{-1} \mathcal{H}_{Q} \phi\right) & =\sum_{j=1}^{n} c_{j} \frac{\delta_{Q}(l)}{\delta_{P}(l)} v_{j}\left(\mathcal{R}_{Q} \varphi\right)(l) \\
& =\sum_{j=1}^{n} c_{j} \frac{\delta_{Q}(l)}{\delta_{P}(l)} \mathcal{R}_{Q}\left(v_{j} \phi\right)(l) \\
& =\sum_{j=1}^{n} c_{j} \frac{1}{\delta_{P}(l)} \mathcal{H}_{Q}\left(v_{j} \phi\right)(l) \quad(l \in L) .
\end{aligned}
$$

Here we note that the above interchange of $v_{j}$ and $\mathcal{R}_{Q}$ is justified by the final assertion of Proposition 2.11. By the first part of the proof, the functions $\delta_{P}^{-1} \mathcal{H}_{Q}\left(v_{j} \phi\right)$ are integrable on $L / H_{L}$. It follows that $u\left(\delta_{P}^{-1} \mathcal{H}_{Q} \phi\right)$ is integrable as well.

We denote by $C_{0}\left(L / H_{L}\right)$ the space of continuous functions $L / H_{L} \rightarrow \mathbb{C}$ which vanish at infinity. Equipped with the sup-norm, this is a Banach space.

Lemma 3.5. The space $L^{1}\left(L / H_{L}\right)^{\infty}$ is contained in $C_{0}\left(L / H_{L}\right)$, with continuous inclusion map.

For a general symmetric space, this result is proved in $\mathrm{KrS12}$. We only need it in the present more restricted setting, which is essentially Euclidean.

Proof. The multiplication map induces a diffeomorphism $L / H_{L} \simeq M / H_{M} \times A_{\mathfrak{q}}$. Since $M / H_{M}$ is compact, it readily follows that

$$
L^{1}\left(L / H_{L}\right)^{\infty} \hookrightarrow C\left(M / H_{M}, L^{1}\left(A_{\mathfrak{q}}\right)^{\infty}\right),
$$

with continuous inclusion map. By the Fourier inversion formula on $A_{\mathfrak{q}}$, combined with application of the Riemann-Lebesgue lemma, $L^{1}\left(A_{\mathfrak{q}}\right)^{\infty} \subseteq C_{0}\left(A_{\mathfrak{q}}\right)$ continuously. Hence, $C\left(M / H_{M}, L^{1}\left(A_{\mathfrak{q}}\right)^{\infty}\right)$ is contained in $C\left(M / H_{M}, C_{0}\left(A_{\mathfrak{q}}\right)\right)$, continuously. Let $C_{b}\left(A_{\mathfrak{q}}\right)$ be the Banach space of bounded continuous functions on $A_{\mathfrak{q}}$, equipped with the sup-norm. Then $C_{0}\left(A_{\mathfrak{q}}\right)$ is a closed subspace of $C_{b}\left(A_{\mathfrak{q}}\right)$. Likewise, $C_{0}\left(L / H_{L}\right)$ is a closed subspace of $C_{b}\left(L / H_{L}\right)$, the Banach space of bounded continuous functions on $L / H_{L}$.

By compactness of $M / H_{M}$, the diffeomorphism mentioned in the beginning of the proof induces a continuous linear isomorphism

$$
\psi: C\left(M / H_{M}, C_{b}\left(A_{\mathfrak{q}}\right)\right) \rightarrow C_{b}\left(L / H_{L}\right) .
$$

It suffices to show that $\psi$ maps the subspace $C\left(M / H_{M}, C_{0}\left(A_{\mathfrak{q}}\right)\right)$ into the closed subspace $C_{0}\left(L / H_{L}\right)$ of $C_{b}\left(L / H_{L}\right)$. This can be achieved by application of a straightforward argument involving the compactness of $M / H_{M}$. 
We recall the definition of the continuous linear map ' $\mathcal{R}_{Q}: L^{1}(G / H) \rightarrow L_{\text {loc }}^{1}(G / H)$ from Corollary 2.10 and note that by Proposition 2.11 this map can be viewed as the continuous linear extension of the restriction of the Radon transform $\mathcal{R}_{Q}$ to $C_{c}^{\infty}(G / H)$.

Theorem 3.6. Let $\phi \in L^{1}(G / H) \cap L_{\mathrm{ds}}^{2}(G / H)$. Then ' $\mathcal{R}_{Q}(\phi)=0$.

Proof. By equivariance and continuity of ' $\mathcal{R}_{Q}$ (see Corollary 2.10) it suffices to prove this result for a $K$-finite function $\phi$. Thus, we may assume that $\phi \in L^{1}(G / H) \cap$ $L_{\mathrm{ds}}^{2}(g / H)_{\vartheta}$, with $\vartheta$ a finite subset of $K^{\wedge}$. It follows from the theory of the discrete series developed in [ŌM84] and vdBS05, Lemma $12.6 \&$ Rem. 12.7] that the space $L_{\mathrm{ds}}^{2}(G / H)_{\vartheta}$ is finite dimensional and consists of smooth functions. It follows that the center of $U(\mathfrak{g})$ acts finitely on $\phi$. In view of vdB87a, Thm. 7.3], we infer that $\phi$ is contained in the $L^{1}$-Schwartz space $\mathscr{C}^{1}(G / H)$ and therefore, so is the $(\mathfrak{g}, K)$ module $V$ generated by $\phi$. In particular, $V$ is contained in $L^{1}(G / H)^{\infty}$ and we see that ${ }^{'} \mathcal{R}_{Q}=\mathcal{R}_{Q}$ on $V$.

We now observe that the assignment $T:\left.\psi \mapsto \mathcal{R}_{Q}(\psi)\right|_{A}$ defines a linear map $V \rightarrow C^{\infty}(A /(A \cap H))$; see Proposition [2.11. Since $L$ normalizes $\mathfrak{n}_{Q}$, we infer that $T$ factors through a map $\bar{T}: V / \mathfrak{n}_{Q} V \rightarrow C^{\infty}(A / A \cap H)$. It is well known that $\operatorname{dim} V / \mathfrak{n}_{Q} V<\infty$; see Wal88, Lemma 4.3.1]. From the equivariance of $\mathcal{R}_{Q}$ it follows that $\bar{T}$ is $U(\mathfrak{a})$-equivariant. Hence, for $\psi \in V$, the function $T(\psi)$ is of exponential polynomial type on $A_{\mathfrak{q}} \simeq A / A \cap H$. By application of Proposition 3.3 and Lemma 3.5 we infer that $\delta_{P}^{-1} \delta_{Q} T(\psi)$ is an exponential polynomial function on $A_{\mathfrak{q}}$ which vanishes at infinity. This implies $T \psi=0$, hence $T(V)=0$. It follows that the map $\psi \mapsto \mathcal{R}_{Q} \psi(e)$ is zero on the closure of $V$ in $L^{1}(G)^{\infty}$, hence on $L_{g} \phi$ for every $g \in G$. We conclude that $\mathcal{R}_{Q} \phi=0$.

Remark 3.7. For Radon transforms associated with minimal $\sigma \theta$-stable parabolic subgroups the analogous result for analytic vectors in $L^{1}(G / H)$ was obtained by a similar a-weight analysis in $\mathrm{Kr} 09$, Thm 4.1]. Let $P_{0} \in \mathscr{P}_{\sigma}\left(A_{\mathfrak{q}}\right)$ be such a parabolic subgroup. Then there exists a parabolic subgroup $P \in \mathscr{P}(A)$ such that $P \subseteq P_{0}$. Since $N_{P_{0}} \simeq N_{P} /\left(N_{P} \cap H\right)$, the Radon transform for $P_{0}$ coincides with $\mathcal{R}_{P}$, and our result implies that the restriction to analytic vectors is unnecessary.

The results in Proposition 3.3 can be improved if only compactly supported smooth functions on $G / H$ are considered. We start by describing the support of the Harish-Chandra transform of a function in terms of the latter's support. To prepare for this, we introduce some notation.

For each $\alpha \in \Sigma \cap \mathfrak{a}_{\mathfrak{q}}^{*}$ the root space $\mathfrak{g}_{\alpha}$ is invariant under the involution $\sigma \theta$ so that the root space admits the decomposition $\mathfrak{g}_{\alpha}=\mathfrak{g}_{\alpha,+} \oplus \mathfrak{g}_{\alpha,-}$ into the \pm 1 -eigenspaces for this involution. Accordingly, for any $Q \in \mathscr{P}(A)$ we define the set

$$
\Sigma(Q)_{-}:=\left\{\alpha \in \Sigma(Q, \sigma \theta): \alpha \in \mathfrak{a}_{\mathfrak{q}}^{*} \Rightarrow \mathfrak{g}_{\alpha,-} \neq 0\right\} .
$$

We define the cone

$$
\Gamma(Q)=\sum_{\alpha \in \Sigma(Q)_{-}} \mathbb{R}_{\geq 0} \operatorname{pr}_{\mathfrak{q}} H_{\alpha}
$$

Here $H_{\alpha}$ denotes the unique element of $\mathfrak{a}$ for which $\alpha=B\left(H_{\alpha}, \cdot\right)$; see (1.4). Furthermore, $\operatorname{pr}_{\mathfrak{q}}: \mathfrak{a} \rightarrow \mathfrak{a}_{\mathfrak{q}}$ denotes the $B$-orthogonal projection. 
Proposition 3.8. Let $C \subseteq \mathfrak{a}_{\mathfrak{q}}$ be compact, convex and invariant under the action of $N_{K \cap H}\left(\mathfrak{a}_{\mathfrak{q}}\right)$. If $\phi \in C_{c}(G / H)$ satisfies

$$
\operatorname{supp}(\phi) \subseteq K \exp (C) \cdot H,
$$

then

$$
\operatorname{supp}\left(\mathcal{H}_{Q} \phi\right) \subseteq M \exp (C+\Gamma(Q)) \cdot H_{L} .
$$

This proposition generalizes AFJS12, Thm. 5.1], which deals with the special case in which $G / H$ is a real hyperbolic space and $Q$ is $\mathfrak{h}$-extreme. See also Kui13, Sect. 4], where similar results are proved for $\sigma \theta$-stable parabolic subgroups.

Proof of Proposition 3.8. Let $\phi \in C_{c}(G / H)$. Assume that $m \in M$ and $a \in A_{\mathfrak{q}}$ are such that $\mathcal{H}_{Q} \phi(m a) \neq 0$. Then

$$
\operatorname{maN}_{Q} \cap K \exp (C) H \neq \emptyset .
$$

Let $\mathfrak{A}_{Q}$ be the map $G \rightarrow \mathfrak{a}_{\mathfrak{q}}$ determined by $g \in K \exp \left(\mathfrak{A}_{Q}(g)\right)(A \cap H) N_{Q}$. Then $\log (a) \in \mathfrak{A}_{Q}(\exp (C) H)$. By BvdB14, Thm. 10.1]

$$
\mathfrak{A}_{Q}(\exp (C) H)=\bigcup_{X \in C} \operatorname{conv}\left(N_{K \cap H}\left(\mathfrak{a}_{\mathfrak{q}}\right) \cdot X\right)+\Gamma(Q) .
$$

Since $C$ is convex and $N_{K \cap H}\left(\mathfrak{a}_{\mathfrak{q}}\right)$-invariant, it follows that the right-hand side equals $C+\Gamma(Q)$. Therefore, $\log (a) \in C+\Gamma(Q)$. The compactness of $C$ and the fact that $\Gamma(Q)$ is closed imply that $C+\Gamma(Q)$ is closed, hence $M \exp (C+\Gamma(Q)) \cdot H_{L}$ is closed. The support of $\mathcal{H}_{Q} \phi$ equals the closure of the subset of $L / H_{L}$ on which $\mathcal{H}_{Q} \phi$ is non-zero, hence

$$
\operatorname{supp}\left(\mathcal{H}_{Q} \phi\right) \subseteq M \exp (C+\Gamma(Q)) \cdot H_{L} .
$$

For a compact subset $U$ of $G / H$, let $C_{U}^{\infty}(G / H)$ be the space of smooth functions on $G / H$ with support contained in $U$, equipped with the usual Fréchet topology. As usual, we equip the space $C_{c}^{\infty}(G / H)$ with the inductive limit topology of the family of spaces $C_{U}^{\infty}(G / H)$, where $U$ runs over all compact subsets of $G / H$.

Proposition 3.9. Let $P \in \mathscr{P}_{\sigma}(A, Q)$. Then $\delta_{P}^{-1} \mathcal{H}_{Q}$ is a continuous linear map $C_{c}^{\infty}(G / H) \rightarrow L^{1}\left(L / H_{L}\right)^{\infty}$.

Proof. Let $\phi \in C_{c}^{\infty}(G / H)$ and let $u \in U(\mathfrak{g})$. Let $c_{j}$ and $v_{j}$ be as in the proof for Proposition 3.3. Then

$$
u\left(\frac{\mathcal{H}_{Q} \phi}{\delta_{P}}\right)=\sum_{j=1}^{n} c_{j} \frac{\mathcal{H}_{Q}\left(v_{j} \phi\right)}{\delta_{P}} .
$$

Let $U$ be a compact subset of $G / H$ such that $\operatorname{supp} \phi \subseteq U$ and let $\vartheta \in C_{c}^{\infty}(G / H)$ be non-negative and equal to 1 on an open neighborhood of $U$. Then

$$
\left|u\left(\frac{\mathcal{H}_{Q} \phi}{\delta_{P}}\right)\right| \leq\left(\sum_{j=1}^{n}\left|c_{j}\right| \sup \left|v_{j} \phi\right|\right) \frac{\mathcal{H}_{Q} \vartheta}{\delta_{P}} .
$$

It follows from Proposition 3.3 that $\delta_{P}^{-1} \mathcal{H}_{Q} \vartheta \in L^{1}\left(L / H_{L}\right)^{\infty}$. From (3.4) we now conclude that $\delta_{P}^{-1} \mathcal{H}_{Q}$ is a continuous linear map $C_{c}^{\infty}(G / H) \rightarrow L^{1}\left(L / H_{L}\right)^{\infty}$. 
We write $\Gamma(Q)^{\circ}$ for the dual cone of $\Gamma(Q)$, i.e.,

$$
\Gamma(Q)^{\circ}=\left\{\lambda \in \mathfrak{a}_{\mathfrak{q}}^{*}: \lambda \geq 0 \text { on } \Gamma(Q)\right\} .
$$

Furthermore, we define

$$
\Omega_{Q}:=\bigcup_{P \in \mathscr{P}_{\sigma}(A, Q)}-\left(\rho_{P}-\rho_{P, \mathfrak{h}}\right)-\Gamma(Q)^{\circ}+i \mathfrak{a}_{\mathfrak{q}}^{*} .
$$

Corollary 3.10. Let $\lambda \in \Omega_{Q}$ and let the character $\chi_{\lambda}: L \rightarrow \mathbb{R}_{>0}$ be given by

$$
\chi_{\lambda}(m a)=a^{\lambda} \quad(m \in M, a \in A) .
$$

Then $\chi_{\lambda} \mathcal{H}_{Q} \phi \in L^{1}\left(L / H_{L}\right)^{\infty}$ for every $\phi \in C_{c}^{\infty}(G / H)$. Moreover, the map

$$
C_{c}^{\infty}(G / H) \rightarrow L^{1}\left(L / H_{L}\right)^{\infty} ; \quad \phi \mapsto \chi_{\lambda} \mathcal{H}_{Q} \phi
$$

is continuous.

Proof. For every $u \in U(\mathfrak{l})$ the function $u\left(\chi_{\lambda} \delta_{P}\right)$ is bounded on $\Gamma(Q)$. The result now follows by application of the Leibniz rule and Propositions 3.8 and 3.9.

\section{Harish-Chandra-Schwartz Functions}

4.1. Definitions. In this subsection we recall some basic facts on the HarishChandra space of $L^{p}$-Schwartz functions on $G / H$ from vdB92, Sect. 17], and give a characterization that will be useful in the next subsection.

Let $\tau: G / H \rightarrow[0, \infty[$ and $\Theta: G / H \rightarrow] 0,1]$ be defined by

$$
\tau(k a H)=\|\log a\|, \quad \Theta(g \cdot H)=\sqrt{\Xi\left(g \sigma(g)^{-1}\right)} .
$$

Here $\Xi$ is Harish-Chandra's bi- $K$-invariant elementary spherical function $\phi_{0}$ on $G$; see, e.g., Var77, p. 329]. Let $V$ be a complete locally convex Hausdorff space and let $\mathcal{N}(V)$ denote the set of continuous seminorms on $V$. Let $1 \leq p<\infty$. A smooth function $\phi: G / H \rightarrow V$ is said to be $L^{p}$-Schwartz if all seminorms

$$
\mu_{u, N, \eta}(\phi):=\sup \Theta^{-\frac{2}{p}}(1+\tau)^{N} \eta(u \phi) \quad(u \in U(\mathfrak{g}), N \in \mathbb{N}, \eta \in \mathcal{N}(V))
$$

are finite. The space of such functions is denoted by $\mathscr{C}^{p}(G / H, V)$. Equipped with the topology induced by the mentioned seminorms, $\mathscr{C}^{p}(G / H, V)$ is a complete locally convex space. Furthermore, it is Fréchet if $V$ is Fréchet.

Let $\mathfrak{v}$ be a $\sigma$ and $\theta$-stable central subalgebra of $\mathfrak{g}$ such that $G={ }^{\circ} G \times \exp (\mathfrak{v})$, where ${ }^{\circ} G=K \exp (\mathfrak{p} \cap[\mathfrak{g}, \mathfrak{g}])$. Define the functions $\Phi_{1}, \Phi_{2}: G \rightarrow[1, \infty[$ by

$$
\begin{gathered}
\Phi_{1}:=1+|\log \circ \Theta|=1-\log \circ \Theta, \\
\Phi_{2}\left(g \exp \left(v_{\mathfrak{h}}+v_{\mathfrak{q}}\right)\right):=\sqrt{1+\left\|v_{\mathfrak{q}}\right\|^{2}} \quad\left(g \in{ }^{\circ} G, v_{\mathfrak{h}} \in \mathfrak{v} \cap \mathfrak{h}, v_{\mathfrak{q}} \in \mathfrak{v} \cap \mathfrak{q}\right) .
\end{gathered}
$$

By [vdB92, Lemma 17.10] there exists a positive constant $C$ such that

$$
C^{-1}(1+\tau) \leq \Phi_{1}+\Phi_{2} \leq C(1+\tau) .
$$

Moreover, $\Phi_{1}$ and $\Phi_{2}$ are real analytic and for every $u \in U(\mathfrak{g})$ there exists a constant $c>0$ such that

$$
\left|u \Phi_{j}\right| \leq c \Phi_{j} \quad(j=1,2) .
$$

The following result is now straightforward. 
Lemma 4.1. Let $\phi: G / H \rightarrow V$ be smooth. Then $\phi \in \mathscr{C}^{p}(G / H, V)$ if and only if all seminorms

$$
\nu_{u, N, \eta}(\phi):=\sup e^{\frac{2}{p} \Phi_{1}}\left(\Phi_{1}+\Phi_{2}\right)^{N} \eta(u \phi) \quad(u \in U(\mathfrak{g}), N \in \mathbb{N}, \eta \in \mathcal{N}(V))
$$

are finite.

We write $\mathscr{C}^{p}(G / H)$ for $\mathscr{C}^{p}(G / H, \mathbb{C})$ and $\nu_{u, N}$ for $\nu_{u, N,|\cdot|}$. For convenience, we suppress the superscript $p$ if $p=2$.

4.2. Domination by $K$-fixed Schwartz functions. We start this subsection with an important result which further on will be applied to reduce the convergence of certain integrals to the case of $K$-finite functions.

Proposition 4.2. There exists a map $\mathscr{C}^{p}(G / H) \rightarrow \mathscr{C}^{p}(G / H)^{K} ; \phi \mapsto \widehat{\phi}$ with the following properties.

(a) $|\phi| \leq \widehat{\phi}$ for all $\phi \in \mathscr{C}^{p}(G / H)$.

(b) Let $\nu$ be a continuous seminorm on $\mathscr{C}^{p}(G / H)$. Then there exist constants $k \in \mathbb{N}$ and $C>0$ such that for all $\phi \in \mathscr{C}^{p}(G / H)$,

$$
\nu(\widehat{\phi}) \leq C \nu_{0, k}(\phi) \text {. }
$$

We first prove two lemmas.

Let $\mathscr{L}\left(\mathbb{R}^{2}\right)$ be the space of locally integrable functions $\mathbb{R}^{2} \rightarrow \mathbb{C}$ which are constant on $\mathbb{R}^{2} \backslash\left[2, \infty\left[{ }^{2}\right.\right.$.

Lemma 4.3. Let $\chi \in C_{c}^{\infty}\left(\mathbb{R}^{2}\right)$ satisfy $\left.\operatorname{supp}(\chi) \subseteq\right]-1,1\left[{ }^{2}\right.$. For every $N \in \mathbb{N}$ there exists a constant $c_{N}>0$ such that for all $S \in \mathscr{L}\left(\mathbb{R}^{2}\right)$,

$$
\sup _{(x, y) \in[1, \infty[2} e^{\frac{2}{p} x}(x+y)^{N}|(\chi * S)(x, y)| \leq c_{N} \sup _{(x, y) \in\left[1, \infty\left[\left[^{2}\right.\right.\right.} e^{\frac{2}{p} x}(x+y)^{N}|S(x, y)| .
$$

Proof. Let $c=\int_{\mathbb{R}^{2}}|\chi(\xi)| d \xi$. Then

$$
|\chi * S(x, y)| \leq c \sup _{(x, y)+]-1,1[2}|S|,
$$

hence

$$
\begin{aligned}
& \sup _{(x, y) \in\left[1, \infty\left[^{2}\right.\right.}\left|e^{\frac{2}{p} x}(x+y)^{N}(\chi * S)(x, y)\right| \\
& \quad \leq c \sup _{(x, y) \in\left[1, \infty\left[^{2}\right.\right.}\left(e^{\frac{2}{p} x}(x+y)^{N} \sup _{(x, y)+]-1,1\left[^{2}\right.}|S|\right) \\
& \quad \leq c \sup _{(u, v) \in] 0, \infty\left[^{2}\right.}\left(e^{\frac{2}{p}(u+1)}(u+v+2)^{N}|S(u, v)|\right) .
\end{aligned}
$$

Since $S$ is constant on $] 0, \infty\left[{ }^{2} \backslash\left[2, \infty\left[{ }^{2}\right.\right.\right.$, the supremum over $] 0, \infty\left[{ }^{2}\right.$ can be replaced by a supremum over $\left[1, \infty\left[{ }^{2}\right.\right.$. Using that

$$
\frac{u+v+2}{u+v} \leq 2 \quad\left(( u , v ) \in \left[1, \infty\left[{ }^{2}\right),\right.\right.
$$

we find

$$
\sup _{(x, y) \in[1, \infty[2}\left|e^{\frac{2}{p} x}(x+y)^{N}(\chi * S)(x, y)\right| \leq c e^{\frac{2}{p}} 2^{N} \sup _{(x, y) \in\left[1, \infty,\left[^{2}\right.\right.}\left(e^{\frac{2}{p} x}(x+y)^{N}|S(x, y)|\right) .
$$

This establishes the estimate. 
For $\phi \in \mathscr{C}^{p}(G / H)$, let $S_{\phi}: \mathbb{R}^{2} \rightarrow \mathbb{R}$ be the function which for $(x, y)$ in the set $[i, i+1[\times[j, j+1[$ with $i, j \in \mathbb{Z}$ is given by

$$
S_{\phi}(x, y)=\sup _{\Phi_{1}^{-1}\left(\left[i, \infty[) \cap \Phi_{2}^{-1}([j, \infty[)\right.\right.}|\phi| .
$$

Note that $\Phi_{j}^{-1}\left(\left[1, \infty[)=\Phi_{j}^{-1}(\mathbb{R})\right.\right.$, so that $S_{\phi} \in \mathscr{L}\left(\mathbb{R}^{2}\right)$. For $\chi \in C_{c}^{\infty}\left(\mathbb{R}^{2}\right)$ we define the smooth function

$$
\widehat{\phi}_{\chi}: G / H \rightarrow \mathbb{C}, \quad x \mapsto\left(\chi * S_{\phi}\right)\left(\Phi_{1}(x), \Phi_{2}(x)\right) .
$$

Since $\Phi_{1}$ and $\Phi_{2}$ are left $K$-invariant, so is the function (4.3).

Lemma 4.4. Let $\chi \in C_{c}^{\infty}\left(\mathbb{R}^{2}\right)$ have support contained in $]-1,1\left[{ }^{2}\right.$.

(a) If $\phi \in \mathscr{C}^{p}(G / H)$, then $\widehat{\phi}_{\chi} \in \mathscr{C}^{p}(G / H)^{K}$.

(b) Let $u \in U(\mathfrak{g})$ be of order $n$. Then for every $N \in \mathbb{N}$ there exists a constant $c_{u, N}>0$ such that

$$
\nu_{u, N}\left(\widehat{\phi}_{\chi}\right) \leq c_{u, N} \nu_{0, N+n}(\phi) \quad\left(\phi \in \mathscr{C}^{p}(G / H)\right) .
$$

Proof. Since the function (4.3) is smooth and left $K$-invariant, it suffices to prove (b). Let $u \in U(\mathfrak{g})$ and let $n$ be the order of $u$. Then by repeated application of the Leibniz and the chain rule it follows that there exists a finite set $F \subseteq U(\mathfrak{g})$ and for every multi-index $\mu$ in two variables, with $|\mu| \leq n$, a polynomial expression $P_{\mu}$ in $\left(v \Phi_{j}: v \in F, j=1,2\right)$, of total degree at most $n$, such that

$$
u \widehat{\phi}_{\chi}=\sum_{|\mu| \leq n} P_{\mu} \cdot \widehat{\phi}_{\partial^{\mu} \chi} \quad\left(\phi \in \mathscr{C}^{p}(G / H)\right)
$$

In view of (4.1) this leads to the existence of a constant $C>0$ such that

$$
\left|u \widehat{\phi}_{\chi}\right| \leq C\left(\Phi_{1}+\Phi_{2}\right)^{n} \sum_{|\mu| \leq n}\left|\widehat{\phi}_{\partial^{\mu} \chi}\right| .
$$

Therefore,

$$
\nu_{u, N}\left(\widehat{\phi}_{\chi}\right) \leq C \sum_{|\mu| \leq n} \nu_{0, N+n}\left(\widehat{\phi}_{\partial^{\mu} \chi}\right) .
$$

Thus, in order to prove the lemma, it suffices to prove that for every $N \in \mathbb{N}$ and $\chi \in C_{c}^{\infty}\left(\mathbb{R}^{2}\right)$ the estimate (4.4) holds for $u=1$.

Let $N \in \mathbb{N}$. Then

$$
\begin{aligned}
\nu_{0, N}\left(\widehat{\phi}_{\chi}\right) & =\sup _{G / H} e^{\frac{2}{p} \Phi_{1}}\left(\Phi_{1}+\Phi_{2}\right)^{N}\left|\left(\chi * S_{\phi}\right) \circ\left(\Phi_{1} \times \Phi_{2}\right)\right| \\
& =\sup _{(x, y) \in[1, \infty[2} e^{\frac{2}{p} x}(x+y)^{N}\left|\chi * S_{\phi}(x, y)\right| .
\end{aligned}
$$

By Lemma 4.3 we now infer the existence of a constant $c_{N}>0$ such that

$$
\nu_{0, N}\left(\widehat{\phi}_{\chi}\right) \leq c_{N} \sup _{(x, y) \in[1, \infty[2} e^{\frac{2}{p} x}(x+y)^{N}\left|S_{\phi}(x, y)\right| .
$$


Let $(x, y) \in\left[1, \infty\left[{ }^{2}\right.\right.$. There exist unique $i, j \in \mathbb{Z}_{\geq 0}$ such that $i \leq x<i+1$ and $j \leq y<j+1$. Then

$$
\begin{aligned}
& e^{\frac{2}{p} x}(x+y)^{N}\left|S_{\phi}(x, y)\right|=e^{\frac{2}{p} x}(x+y)^{N}\left(\sup _{\Phi_{1}^{-1}\left(\left[i, \infty[) \cap \Phi_{2}^{-1}([j, \infty[)\right.\right.}|\phi|\right) \\
& \quad \leq \sup _{\Phi_{1}^{-1}\left(\left[i, \infty[) \cap \Phi_{2}^{-1}([j, \infty[)\right.\right.} e^{\frac{2}{p}\left(\Phi_{1}+1\right)}\left(\Phi_{1}+\Phi_{2}+2\right)^{N}|\phi| \\
& \quad \leq \sup e^{\frac{2}{p}\left(\Phi_{1}+1\right)}\left(\Phi_{1}+\Phi_{2}+2\right)^{N}|\phi| \\
& \quad \leq \sup e^{\frac{2}{p}\left(\Phi_{1}+1\right)} 2^{N}\left(\Phi_{1}+\Phi_{2}\right)^{N}|\phi|=2^{N} e^{\frac{2}{p}} \nu_{0, N}(\phi) .
\end{aligned}
$$

Combining this estimate with (4.5) we obtain the estimate of (b) with $u=1$.

Proof of Proposition 4.2. Let $\chi \in C_{c}^{\infty}(] 0,1\left[^{2}\right)$ be a non-negative function such that $\int_{\mathbb{R}} \chi(x) d x=1$. If $\phi \in \mathscr{C}^{p}(G / H)$, then $\widehat{\phi}_{\chi} \in C^{\infty}(G / H)^{K}$. Moreover, since $S_{\phi}$ is decreasing in both variables, it follows from the condition on supp $\chi$ that

$$
\widehat{\phi}=\left(\chi * S_{\phi}\right) \circ\left(\Phi_{1} \times \Phi_{1}\right) \geq S_{\phi} \circ\left(\Phi_{1} \times \Phi_{1}\right) \geq|\phi| .
$$

This establishes (a).

In order to complete the proof, it suffices to prove (b) for $\nu=\nu_{u, N}$, with $u \in U(\mathfrak{g})$ of order at most $n$ and for $N \in \mathbb{N}$. Let $k=N+n$. Then the estimate (4.2) follows by application of Lemma 4.4 .

For the application of Proposition 4.2 we will need the following useful lemma.

Lemma 4.5. Let $\psi \in \mathscr{C}(G / H)^{K}$ be non-negative. Then there exists a monotonically increasing sequence $\left(\psi_{j}\right)_{j \in \mathbb{N}}$ in $C_{c}^{\infty}(G / H)^{K}$ such that $\psi_{j} \rightarrow \psi$ in $\mathscr{C}(G / H)^{K}$, for $j \rightarrow \infty$.

Proof. For $r>0$ we define $B(r):=\{x \in G / H: \tau(x) \leq r\}$. By vdB87b, Lemma 2.2] and its proof, there exists a sequence of functions $g_{j} \in C_{c}^{\infty}(G / H)$ such that the following conditions are fulfilled:

(1) $0 \leq g_{j} \leq g_{j+1} \leq 1$, for $j \geq 0$;

(2) $g_{j}=1$ on $B(j)$ and $\operatorname{supp} g_{j} \subseteq B(j+1)$, for $j \geq 0$;

(3) for every $u \in U(\mathfrak{g})$ there exists $C_{u}>0$ such that $\sup _{G / H}\left|L_{u} g_{j}\right| \leq C_{u}$ for all $j \geq 1$.

By using the argument of $\operatorname{Var} 77$, Thm. 2, p. 343] one now readily checks that the sequence $\psi_{j}=g_{j} \psi$ satisfies our requirements.

Proposition 4.2 now leads to the following results concerning the Radon and Harish-Chandra transforms.

Proposition 4.6. Assume that the restriction of $\mathcal{H}_{Q}$ to $C_{c}^{\infty}(G / H)^{K}$ extends to a continuous linear map $\mathscr{C}(G / H)^{K} \rightarrow C\left(L / H_{L}\right)$. Then $\mathcal{R}_{Q}$ extends to a continuous linear map

$$
\mathcal{R}_{Q}: \mathscr{C}(G / H) \rightarrow C^{\infty}\left(G / N_{Q}\right)
$$

and for every $\phi \in \mathscr{C}(G / H)$,

$$
\mathcal{R}_{Q} \phi(g)=\int_{N_{Q} / H_{N_{Q}}} \phi(g n) d n \quad(g \in G)
$$


with absolutely convergent integrals. Furthermore, the restriction of $\mathcal{H}_{Q}$ to $C_{c}^{\infty}(G / H)$ extends to a continuous linear map

$$
\mathcal{H}_{Q}: \mathscr{C}(G / H) \rightarrow C^{\infty}\left(L / H_{L}\right)
$$

and for every $\phi \in \mathscr{C}(G / H)$,

$$
\mathcal{H}_{Q} \phi(l)=\delta_{Q}(l) \int_{N_{Q} / H_{N_{Q}}} \phi(l n) d n \quad(l \in L)
$$

with absolutely convergent integrals.

Proof. Since

$$
\mathcal{R}_{Q} \phi(k a n)=\delta_{Q}(a)^{-1} \mathcal{H}_{Q} \phi(a) \quad\left(\phi \in C_{c}^{\infty}(G / H)^{K}, k \in K, a \in A, n \in N_{Q}\right),
$$

it follows from the assumption in the proposition that the restriction of $\mathcal{R}_{Q}$ to $C_{c}^{\infty}(G / H)^{K}$ extends to a continuous linear map $\mathscr{C}(G / H)^{K} \rightarrow C\left(G / N_{Q}\right)^{K}$.

Let $\psi \in \mathscr{C}(G / H)^{K}$ be non-negative. We claim that $\mathcal{R}_{Q} \psi$ is given by (4.6). To see this, let $\left(\psi_{j}\right)_{j \in \mathbb{N}}$ be a monotonically increasing sequence as in Lemma 4.5. Then for every $g \in G$, we have

$$
\mathcal{R}_{Q} \psi(g)=\lim _{j \rightarrow \infty} \mathcal{R}_{Q} \psi_{j}(g)=\lim _{j \rightarrow \infty} \int_{N_{Q} / H_{N_{Q}}} \psi_{j}(g n) d n .
$$

Since the sequence $\psi_{j}$ is monotonically increasing, the monotone convergence theorem implies that (4.6) holds and that the integral is absolutely convergent, for every $g \in G$.

By Proposition 4.2 every element of $\mathscr{C}(G / H)$ can be dominated by an element of $\mathscr{C}(G / H)^{K}$. Hence, for every $\phi \in \mathscr{C}(G / H)$ and $g \in G$ the integral in (4.6) is absolutely convergent. For $\phi \in \mathscr{C}(G / H)$ we now define $\mathcal{R}_{Q} \phi$ and $\mathcal{H}_{Q} \phi$ by (4.6) and (4.7), respectively. To finish the proof of the proposition, it suffices to show that $\mathcal{R}_{Q} \phi$ is smooth and that the map $\mathcal{R}_{Q}: \mathscr{C}(G / H) \rightarrow C^{\infty}\left(G / N_{Q}\right)$ is continuous.

By assumption, there exists a continuous seminorm $\nu$ on $\mathscr{C}(G / H)$ such that for all $\psi \in \mathscr{C}(G / H)^{K}$,

$$
\sup _{G}\left|\mathcal{R}_{Q}(\psi)\right| \leq \nu(\psi)
$$

Let $\phi \mapsto \widehat{\phi}$ be a map $\mathscr{C}(G / H) \rightarrow \mathscr{C}(G / H)^{K}$ as in Proposition 4.2, with $p=2$. Let $C>0$ and $n \in \mathbb{N}$ be associated with $\nu$ as in the mentioned proposition. Then it follows that for all $\phi \in \mathscr{C}(G / H)$,

$$
\left|\mathcal{R}_{Q}(\phi)\right| \leq \mathcal{R}_{Q}(|\phi|) \leq \mathcal{R}_{Q}(\widehat{\phi}) \leq \nu(\widehat{\phi}) \leq C \nu_{0, n}(\phi)
$$

We thus see that $\mathcal{R}_{Q}$ defines a continuous linear map

$$
\mathcal{R}_{Q}: \mathscr{C}(G / H) \rightarrow C\left(G / N_{Q}\right) .
$$

Since this map intertwines the left $G$-actions, whereas the left regular representation of $G$ in $\mathscr{C}(G / H)$ is smooth, it follows that $\mathcal{R}_{Q}$ maps continuously into the space of smooth vectors of $C\left(G / N_{Q}\right)$, which equals $C^{\infty}\left(G / N_{Q}\right)$ as a topological linear space. 


\section{FOURIER TRANSFORMS}

5.1. Densities and a Fubini theorem. In this section we introduce some notation related to densities on homogeneous spaces. Further details can be found in vdBK14, App. A]. For our purposes it is more convenient to consider rightquotients $S / T$ of Lie groups instead of left-quotients $T \backslash S$, which we used in the aforementioned article.

If $V$ is a real finite dimensional vector space, then we write $\mathscr{D}_{V}$ for the space of complex-valued densities on $V$, i.e., the 1-dimensional complex vector space of functions $\omega: \wedge^{\text {top }}(V) \rightarrow \mathbb{C}$ transforming according to the rule

$$
\omega(t v)=|t| \omega(v) \quad\left(t \in \mathbb{R}, v \in \wedge^{\text {top }} V\right) .
$$

For a Lie group $S$ and a closed subgroup $T$, let $\Delta_{S / T}: T \rightarrow \mathbb{R}_{+}$be the positive character given by

$$
\Delta_{S / T}(t)=\left|\operatorname{det} \operatorname{Ad}_{S}(t)_{\mathfrak{s} / \mathfrak{t}}\right|^{-1} \quad(t \in T),
$$

where $\operatorname{Ad}_{S}(t)_{\mathfrak{s} / \mathfrak{t}} \in \mathrm{GL}(\mathfrak{s} / \mathfrak{t})$ denotes the map induced by the adjoint map $\operatorname{Ad}_{G}(t) \in$ $\mathrm{GL}(\mathfrak{s})$. We denote by $C\left(S: T: \Delta_{S / T}\right)$ the space of continuous functions $f: S \rightarrow \mathbb{C}$ transforming according to the rule

$$
f(s t)=\Delta_{S / T}(t)^{-1} f(s) \quad(s \in S, t \in T) .
$$

We denote by $\mathscr{M}(G: L: \xi)$ the space of measurable functions $f: G \rightarrow \mathbb{C}$ transforming according to the same rule.

Given $f \in C\left(S: T: \Delta_{S / T}\right)$ and $\omega \in \mathscr{D}_{\mathfrak{s} / \mathfrak{t}}$, we denote by $f_{\omega}$ the continuous density on $S / T$ determined by

$$
f_{\omega}(s)=f(s) d l_{s}(e)^{-1 *} \omega \quad(s \in S) .
$$

We fix non-zero elements $\omega_{S / U} \in \mathscr{D}_{\mathfrak{s} / \mathfrak{u}}, \omega_{T / U} \in \mathscr{D}_{\mathfrak{t} / \mathfrak{u}}$ and $\omega_{S / T} \in \mathscr{D}_{\mathfrak{s} / \mathfrak{t}}$ such that

$$
\omega_{T / U} \otimes \omega_{S / T}=\omega_{S / U}
$$

with respect to the identification determined by the short exact sequence

$$
0 \rightarrow \mathfrak{t} / \mathfrak{u} \rightarrow \mathfrak{s} / \mathfrak{u} \rightarrow \mathfrak{s} / \mathfrak{t} \rightarrow 0 .
$$

See equation (A.10) and the subsequent text in vdBK14 App. A]. We then have the following Fubini theorem [vdBK14, Thm. A.8].

Theorem 5.1. Let $\phi \in \mathscr{M}\left(S: U: \Delta_{S / U}\right)$ and let $\phi_{\omega_{S / U}}$ be the associated measurable density on $S / U$. Then the following assertions (a) and (b) are equivalent.

(a) The density $\phi_{\omega_{S / U}}$ is absolutely integrable.

(b) There exists a right T-invariant subset $\mathscr{Z}$ of measure zero in $S$ such that

(1) for every $x \in S \backslash \mathscr{Z}$, the integral

$$
I_{x}(\phi)=\int_{T / U \ni[t]} \Delta_{S / T}(t) \phi(x t) d l_{t}([e])^{-1 *} \omega_{T / U},
$$

is absolutely convergent;

(2) the function $I(\phi): x \mapsto I_{x}(\phi)$ belongs to $\mathscr{M}\left(S: T: \Delta_{S / T}\right)$;

(3) the associated density $I(\phi)_{\omega_{S / T}}$ is absolutely integrable.

Furthermore, if any of the conditions (a) or (b) are fulfilled, then

$$
\int_{S / U} \phi_{\omega_{S / U}}=\int_{S / T} I(\phi)_{\omega_{S / T}}
$$


5.2. Eisenstein integrals. We start by recalling notation, definitions and results from $\mathrm{vdBK} 14$.

Let $\left(\tau, V_{\tau}\right)$ be a finite dimensional representation of $K$. We write $C^{\infty}(G / H: \tau)$ for the space of smooth $V_{\tau}$-valued functions $\phi$ on $G / H$ that satisfy the transformation property

$$
\phi(k x)=\tau(k) \phi(x) \quad(k \in K, x \in G / H) .
$$

We further write $C_{c}^{\infty}(G / H: \tau)$ and $\mathscr{C}(G / H: \tau)$ for the subspaces of $C^{\infty}(G / H: \tau)$ consisting of compactly supported functions and $L^{2}$-Schwartz functions, respectively.

Let $W\left(\mathfrak{a}_{\mathfrak{q}}\right)$ be the Weyl group of the root system of $\mathfrak{a}_{\mathfrak{q}}$ in $\mathfrak{g}$. Then

$$
W\left(\mathfrak{a}_{\mathfrak{q}}\right)=N_{K}\left(\mathfrak{a}_{\mathfrak{q}}\right) / Z_{K}\left(\mathfrak{a}_{\mathfrak{q}}\right) .
$$

Let $W_{K \cap H}\left(\mathfrak{a}_{\mathfrak{q}}\right)$ be the subgroup of $W\left(\mathfrak{a}_{\mathfrak{q}}\right)$ consisting of elements that can be realized in $N_{K \cap H}\left(\mathfrak{a}_{\mathfrak{q}}\right)$. We choose a set $\mathscr{W}$ of representatives for $W\left(\mathfrak{a}_{\mathfrak{q}}\right) / W_{K \cap H}\left(\mathfrak{a}_{\mathfrak{q}}\right)$ in $N_{K}\left(\mathfrak{a}_{\mathfrak{q}}\right) \cap N_{K}\left(\mathfrak{a}_{\mathfrak{h}}\right)$ such that $e \in \mathscr{W}$. This is possible because of the following lemma.

Lemma 5.2. $N_{K}\left(\mathfrak{a}_{\mathfrak{q}}\right)=\left(N_{K}\left(\mathfrak{a}_{\mathfrak{q}}\right) \cap N_{K}\left(\mathfrak{a}_{\mathfrak{h}}\right)\right) Z_{K}\left(\mathfrak{a}_{\mathfrak{q}}\right)$.

This result can be found in [Ros79, p. 165]. For the reader's convenience we give the concise proof.

Proof. It is clear that $\left(N_{K}\left(\mathfrak{a}_{\mathfrak{q}}\right) \cap N_{K}\left(\mathfrak{a}_{\mathfrak{h}}\right)\right) Z_{K}\left(\mathfrak{a}_{\mathfrak{q}}\right) \subseteq N_{K}\left(\mathfrak{a}_{\mathfrak{q}}\right)$. To prove the other inclusion, assume that $k \in N_{K}\left(\mathfrak{a}_{\mathfrak{q}}\right)$. Then $\operatorname{Ad}\left(k^{-1}\right) \mathfrak{a}_{\mathfrak{h}}$ is a maximal abelian subspace of $Z_{\mathfrak{g}}\left(\mathfrak{a}_{\mathfrak{q}}\right) \cap \mathfrak{p}$. Each such maximal abelian subspace is conjugate to $\mathfrak{a}_{\mathfrak{h}}$ by an element from $Z_{G}\left(\mathfrak{a}_{\mathfrak{q}}\right) \cap K$, i.e., there exists a $k^{\prime} \in Z_{K}\left(\mathfrak{a}_{\mathfrak{q}}\right)$ such that $\operatorname{Ad}\left(k^{\prime}\right) \operatorname{Ad}\left(k^{-1}\right) \mathfrak{a}_{\mathfrak{h}}=\mathfrak{a}_{\mathfrak{h}}$. Note that $k^{\prime} k^{-1} \in N_{K}\left(\mathfrak{a}_{\mathfrak{q}}\right) \cap N_{K}\left(\mathfrak{a}_{\mathfrak{h}}\right)$. Hence,

$$
k=\left(k k^{\prime-1}\right) k^{\prime} \in\left(N_{K}\left(\mathfrak{a}_{\mathfrak{q}}\right) \cap N_{K}\left(\mathfrak{a}_{\mathfrak{h}}\right)\right) Z_{K}\left(\mathfrak{a}_{\mathfrak{q}}\right) .
$$

We define

$$
M_{0}:=Z_{K}\left(\mathfrak{a}_{\mathfrak{q}}\right) \exp \left(\mathfrak{p} \cap\left[Z_{\mathfrak{g}}\left(\mathfrak{a}_{\mathfrak{q}}\right), Z_{\mathfrak{g}}\left(\mathfrak{a}_{\mathfrak{q}}\right)\right]\right) .
$$

If $P_{0} \in \mathscr{P}\left(A_{\mathfrak{q}}\right)$, then $M_{0} A$ is a Levi subgroup of $P_{0}$. We write $\mathfrak{m}_{0 n}$ for the direct sum of the non-compact ideals of $\mathfrak{m}_{0}$. The associated connected subgroup of $M_{0}$ is denoted by $M_{0 n}$.

We denote by $\tau_{M}$ the restriction of $\tau$ to $M$. Since $M$ is a subgroup of $M_{0} \cap K$, it normalizes $M_{0 n} \cap K$, so that $\left(V_{\tau}\right)^{M_{0 n} \cap K}$ is an $M$-invariant subspace of $V_{\tau}$. The restriction of $\tau_{M}$ to this subspace is denoted by $\tau_{M}^{0}$. We define

$$
\mathscr{A}_{M, 2}(\tau):=\bigoplus_{v \in \mathscr{W}} C^{\infty}\left(M / M \cap v H v^{-1}: \tau_{M}^{0}\right) .
$$

Each component in the sum is finite dimensional and thus a Hilbert space equipped with the restriction of the inner product of $L^{2}\left(M / \cap v H v^{-1}, V_{\tau}\right)$; the direct sum is equipped with the direct sum Hilbert structure, and thus becomes a finite dimensional Hilbert space.

If $\psi \in \mathscr{A}_{M, 2}(\tau)$, we accordingly write $\psi_{v}$ for the component of $\psi$ in the space $C^{\infty}\left(M / M \cap v H v^{-1}: \tau_{M}^{0}\right)$.

Let $Q \in \mathscr{P}(A)$. For $v \in \mathscr{W}$ we define the parabolic subgroup $Q^{v} \in \mathscr{P}(A)$ by

$$
Q^{v}:=v^{-1} Q v \text {. }
$$


For each $v \in \mathscr{W}$ we choose a positive density

$$
\omega_{H / H_{Q^{v}}} \in \mathscr{D}_{\mathfrak{h} / \mathfrak{h}_{Q^{v}}}
$$

as follows. Fix positive densities $\omega_{G / H} \in \mathscr{D}_{\mathfrak{g} / \mathfrak{h}}$ and $\omega_{G / H_{L}} \in \mathscr{D}_{\mathfrak{g} / \mathfrak{h}_{L}}$. Furthermore, let $\omega_{v} \in \mathscr{D}_{\mathfrak{n}_{Q^{v}} \cap \mathfrak{h}}$ be the positive density that corresponds to the Haar measure on $N_{Q^{v}} \cap H$, which was chosen to be the push-forward of the Lebesgue measure on $\mathfrak{n}_{Q^{v}} \cap \mathfrak{h}$ along the exponential map (see text below Lemma 2.1). Then we choose $\omega_{H / H_{Q} v}$ to be the unique density such that

$$
\omega_{G / H} \otimes \omega_{H / H_{Q^{v}}} \otimes \omega_{v}=\omega_{G / H_{L}} .
$$

The inner product $\left.B\right|_{\mathfrak{a}_{\mathfrak{q}}}$ on $\mathfrak{a}_{\mathfrak{q}}$ induces a linear isomorphism $B: \mathfrak{a}_{\mathfrak{q}} \rightarrow \mathfrak{a}_{\mathfrak{q}}^{*}$. If $Q \in \mathscr{P}(A)$, we define the cone $\Gamma(Q) \subseteq \mathfrak{a}_{\mathfrak{q}}$ as in (3.3). Then $B(\Gamma(Q))$ equals the cone spanned by the elements $\alpha+\sigma \theta \alpha$, with $\alpha \in \Sigma(Q)_{-}$; see (3.2). Let $\Omega_{Q} \subseteq \mathfrak{a}_{\mathfrak{q}}$ be defined as in (3.5) and let $\widehat{\Omega}_{Q}$ denote its hull in $\mathfrak{a}_{\mathfrak{q C}}$ with respect to the functions $\operatorname{Re}\langle\cdot, \alpha\rangle$ with $\alpha \in \Sigma\left(\mathfrak{a}_{\mathfrak{q}}\right) \cap B(\Gamma(Q))$, i.e.,

$$
\widehat{\Omega}_{Q}:=\left\{\lambda \in \mathfrak{a}_{\mathfrak{q} \mathbb{C}}^{*}: \operatorname{Re}\langle\lambda, \alpha\rangle \leq \sup \operatorname{Re}\left\langle\Omega_{Q}, \alpha\right\rangle \forall \alpha \in \Sigma\left(\mathfrak{a}_{\mathfrak{q}}\right) \cap B(\Gamma(Q))\right\} .
$$

Since $\langle\alpha, \lambda\rangle \leq 0$ for all $\alpha \in \Sigma\left(\mathfrak{a}_{\mathfrak{q}}\right) \cap B(\Gamma(Q))$ and $\lambda \in-\Gamma(Q)^{\circ}$, it follows that we can describe the given hull by means of inequalities as follows:

$$
\widehat{\Omega}_{Q}=\left\{\lambda \in \mathfrak{a}_{\mathfrak{q} \mathbb{C}}^{*}: \operatorname{Re}\langle\lambda, \alpha\rangle \leq \max _{P \in \mathscr{P}_{\sigma}(A, Q)}\left\langle-\rho_{P}, \alpha\right\rangle \forall \alpha \in \Sigma\left(\mathfrak{a}_{\mathfrak{q}}\right) \cap B(\Gamma(Q))\right\} .
$$

We define the following closed subsets of $\mathfrak{a}_{\mathfrak{q C}}$,

$$
\Upsilon_{Q}=\bigcap_{v \in \mathscr{W}} v \Omega_{v^{-1} Q v}, \quad \widehat{\Upsilon}_{Q}=\bigcap_{v \in \mathscr{W}} v \widehat{\Omega}_{v^{-1} Q v} .
$$

Given $v \in \mathscr{W}$ we will use the notation

$$
{ }^{v} H:=v H v^{-1} \quad \text { and } \quad{ }^{v} H_{Q}:={ }^{v} H \cap Q .
$$

Furthermore, we define the density ${ }^{v} \omega_{v}$ on ${ }^{v} \mathfrak{h} /{ }^{v} \mathfrak{h}_{Q}$ by

$$
{ }^{v} \omega:=\operatorname{Ad}\left(v^{-1}\right)^{*} \omega_{H / H_{Q^{v}}} .
$$

Given $\psi_{v} \in C^{\infty}\left(M /{ }^{v} H \cap M: \tau_{M}^{0}\right)$ and $\lambda \in \Upsilon_{Q}$ we define the function $\psi_{v, Q, \lambda}: G \rightarrow$ $V_{\tau}$ by

$$
\psi_{v, Q, \lambda}(k m a n)=a^{\lambda-\rho_{Q}-\rho_{Q, \mathfrak{h}}} \tau(k) \psi_{v}(m) \quad\left(k \in K, m \in M, a \in A, n \in N_{Q}\right) .
$$

Then for every $x \in G$ the function

$$
y \mapsto \psi_{v, Q, \lambda}(x y) d l_{y}(e)^{-1 *}\left({ }^{v} \omega\right)
$$

defines a $V_{\tau}$-valued density on ${ }^{v} H /{ }^{v} H_{Q}$, which is integrable by vdBK14, Prop. 8.2].

We define

$$
E_{v H v^{-1}}\left(Q: \psi_{v}: \lambda\right): G \rightarrow V_{\tau}
$$

for $x \in G$ by

$$
\begin{aligned}
E_{v H v^{-1}}\left(Q: \psi_{v}: \lambda\right)(x) & =\int_{v_{H /{ }^{v} H_{Q}}} \psi_{v, Q, \lambda}(x y) d l_{y}(e)^{-1 *}\left({ }^{v} \omega\right) \\
& =\int_{H / H_{Q^{v}}} \psi_{v, Q, \lambda}\left(x v h v^{-1}\right) d l_{h}(e)^{-1 *} \omega_{H^{\prime} H_{Q^{v}}}
\end{aligned}
$$

For $\psi \in \mathscr{A}_{M, 2}$ and $\lambda \in \Upsilon_{Q}$ we define the Eisenstein integral

$$
E(Q: \psi: \lambda)=E_{\tau}(Q: \psi: \lambda): G \rightarrow V_{\tau}
$$


by

$$
E(Q: \psi: \lambda)(x)=\sum_{v \in \mathscr{W}} E_{v H v^{-1}}\left(Q: \operatorname{pr}_{v} \psi: \lambda\right)\left(x v^{-1}\right) \quad(x \in G)
$$

It is readily verified that this function belongs to $C^{\infty}(G / H: \tau)$. We note that the map $E(Q: \psi: \cdot)$ extends to a meromorphic $C^{\infty}(G / H: \tau)$-valued function on $\mathfrak{a}_{\mathfrak{q} \mathbb{C}}^{*}$ and is holomorphic on an open neighborhood of $\widehat{\Upsilon}_{Q}$; see [vdBK14, Cor. 8.5].

Let $Q_{1}, Q_{2} \in \mathscr{P}(A)$. Then there exists a unique meromorphic $\operatorname{End}\left(\mathscr{A}_{M, 2}(\tau)\right)$ valued function $C\left(Q_{2}: Q_{1}: \cdot\right)$ on $\mathfrak{a}_{\mathfrak{q} \mathbb{C}}^{*}$ such that

$$
E\left(Q_{2}: \psi: \lambda\right)=E\left(Q_{1}: C\left(Q_{1}: Q_{2}: \lambda\right) \psi: \lambda\right)
$$

for generic $\lambda \in \mathfrak{a}_{\mathfrak{q} \mathfrak{c}}^{*}$; see $\operatorname{vdBK14}$, Cor. 8.14].

In order to describe the relation of these Eisenstein integrals with those defined in terms of a parabolic subgroup from the set $\mathscr{P}_{\sigma}\left(A_{\mathfrak{q}}\right)$ (see the text preceding (1.2) ), we need to introduce a bit more notation.

Let $\widehat{M}_{0}$ denote the collection of (equivalence classes of) finite dimensional irreducible unitary representations of $M_{0}$. For $\xi \in \widehat{M}_{0}$ and $v \in \mathscr{W}$ we define the finite dimensional Hilbert space

$$
V(\xi, v):=\mathscr{H}_{\xi}^{M_{0} \cap v H v^{-1}} .
$$

The formal direct sum of these gives a finite dimensional Hilbert space

$$
V(\xi):=\bigoplus_{v \in \mathscr{W}} V(\xi, v)
$$

We define $C(K: \xi: \tau)$ to be the space of functions $f: K \rightarrow \mathscr{H}_{\xi} \otimes V_{\tau}$ transforming according to the rule:

$$
f\left(m k_{0} k\right)=\left(\xi(m) \otimes \tau(k)^{-1}\right) f\left(k_{0}\right), \quad\left(k, k_{0} \in K, m \in M_{0}\right) .
$$

Let $\bar{V}(\xi)$ denote the conjugate space of $V(\xi)$ and consider the natural map

$$
T \mapsto \psi_{T}, \quad C(K: \xi: \tau) \otimes \bar{V}(\xi) \rightarrow \mathscr{A}_{M, 2}(\tau),
$$

which for $v \in \mathscr{W}$ and $T=f \otimes \eta \in C(K: \xi: \tau) \otimes \bar{V}(\xi)$ is given by

$$
\left(\psi_{T}\right)_{v}(m)=\left\langle f(e), \xi(m) \eta_{v}\right\rangle_{\xi} \quad\left(m \in M_{0}\right) .
$$

Then the sum of the maps $T \mapsto(\operatorname{dim} \xi)^{\frac{1}{2}} \psi_{T}$ over all $\xi \in \widehat{M}_{0}$ gives a surjective isometry

$$
\bigoplus_{\xi \in \widehat{M}_{0}} C(K: \xi: \tau) \otimes \bar{V}(\xi) \stackrel{\simeq}{\longrightarrow} \mathscr{A}_{M, 2}(\tau) ;
$$

see $\mathrm{vdBS97b}$, Lemma 3]. Note that only finitely many terms in the direct sum are non-zero.

Now let $Q \in \mathscr{P}(A)$ and $R \in \mathscr{P}_{\sigma}\left(A_{\mathfrak{q}}\right)$. Then we define the $C$-functions $C_{R \mid Q}(s: \cdot)$ for $s \in W\left(\mathfrak{a}_{\mathfrak{q}}\right)$ as in vdBK14, Thm. 8.13]. These are $\operatorname{End}\left(\mathscr{A}_{M, 2}(\tau)\right)$-valued meromorphic functions on $\mathfrak{a}_{\mathfrak{q} \mathbb{C}}^{*}$, with meromorphic inverses. Moreover, by uniqueness of asymptotics, they are uniquely determined by the requirement that

$$
E(Q: \psi: \lambda)(a v) \sim \sum_{s \in W\left(\mathfrak{a}_{\mathfrak{q}}\right)} a^{s \lambda-\rho_{R}}\left[C_{R \mid Q}(s: \lambda) \psi\right]_{v}(e), \quad\left(a \rightarrow \infty \text { in } A_{\mathfrak{q}}^{+}(R)\right)
$$

for all $\psi \in \mathscr{A}_{M, 2}(\tau), v \in \mathscr{W}$ and generic $\lambda \in i \mathfrak{a}_{\mathfrak{q}}^{*}$. 
For $P_{0} \in \mathscr{P}_{\sigma}\left(A_{\mathfrak{q}}\right)$ and $\psi \in \mathscr{A}_{M, 2}(\tau)$ we denote by $E\left(P_{0}: \psi: \lambda\right)$ the Eisenstein integral as defined in vdBS97b, Sect. 2]. Then $\lambda \mapsto E\left(P_{0}: \psi: \lambda\right)$ is a meromorphic function on $\mathfrak{a}_{\mathfrak{q} \mathbb{C}}^{*}$ with values in $C^{\infty}(G / H: \tau)$. Given $R \in \mathscr{P}_{\sigma}\left(A_{\mathfrak{q}}\right)$ the $C$-functions

$$
C_{R \mid P_{0}}(s: \lambda) \in \operatorname{End}\left(\mathscr{A}_{M, 2}(\tau)\right),
$$

for $s \in W\left(\mathfrak{a}_{\mathfrak{q}}\right)$ are defined as in vdBS97b, Eqn. (46)]. These are meromorphic functions with values in $\operatorname{End}\left(\mathscr{A}_{M, 2}(\tau)\right)$ and with meromorphic inverses. Moreover, they are uniquely determined by the asymptotic behavior of the Eisenstein integral $E\left(P_{0}: \psi: \lambda\right)$, described by (5.9) with everywhere $Q$ replaced by $P_{0}$.

Lemma 5.3. Let $P_{0} \in \mathscr{P}_{\sigma}\left(A_{\mathfrak{q}}\right)$ and assume that $P \in \mathscr{P}(A)$ is $\mathfrak{q}$-extreme, and satisfies $P \subseteq P_{0}$. Then for generic $\lambda \in \mathfrak{a}_{\mathfrak{q} \mathfrak{c}}^{*}$,

$$
E\left(P_{0}: \psi: \lambda\right)=E(P: \psi: \lambda) \text {. }
$$

Furthermore, for all $R \in \mathscr{P}_{\sigma}\left(A_{\mathfrak{q}}\right), s \in W\left(\mathfrak{a}_{\mathfrak{q}}\right)$ and generic $\lambda \in \mathfrak{a}_{\mathfrak{q} \mathbb{C}}^{*}$,

$$
C_{R \mid P_{0}}(s: \lambda)=C_{R \mid P}(s: \lambda) \text {. }
$$

Proof. The first assertion is made in vdBK14, Cor. 8.6]. The second assertion follows by uniqueness of asymptotics.

For $\psi \in \mathscr{A}_{M, 2}(\tau)$ we define the normalized Eisenstein integral $E^{\circ}\left(\bar{P}_{0}: \psi: \cdot\right)$ as in vdBS97b, Sect. 5,6]. It is a meromorphic $C^{\infty}(H / H: \tau)$-valued function of $\lambda \in \mathfrak{a}_{\mathfrak{q} \mathbb{C}}^{*}$. Furthermore, for any $R \in \mathscr{P}_{\sigma}\left(A_{\mathfrak{q}}\right)$ we have

$$
E(R: \psi: \lambda)=E^{\circ}\left(\bar{P}_{0}: C_{\bar{P}_{0} \mid R}(1: \lambda) \psi: \lambda\right) ;
$$

see vdBS97b, Eqn. (58)].

Lemma 5.4. Let $Q \in \mathscr{P}(A)$ and $P_{0} \in \mathscr{P}_{\sigma}\left(A_{\mathfrak{q}}\right)$. Then

$$
E(Q: \psi: \lambda)=E^{\circ}\left(\bar{P}_{0}: C_{\bar{P}_{0} \mid Q}(1: \lambda) \psi: \lambda\right)
$$

for all $\psi \in \mathscr{A}_{M, 2}(\tau)$ and generic $\lambda \in \mathfrak{a}_{\mathfrak{q} \mathbb{C}}^{*}$.

Proof. Let $P \in \mathscr{P}_{\sigma}(A)$ be such that $P_{0} \supseteq P$. Then it follows from (5.6) and (5.10) that

$$
E(Q: \psi: \lambda)=E(P: C(P: Q: \lambda) \psi: \lambda)=E\left(P_{0}: C(P: Q: \lambda) \psi: \lambda\right) .
$$

Using (5.12) with $R=\bar{P}_{0}$, we infer that

$$
E(Q: \psi: \lambda)=E^{\circ}\left(\bar{P}_{0}: C_{\bar{P}_{0} \mid P_{0}}(1: \lambda) C(P: Q: \lambda) \psi: \lambda\right) .
$$

By application of (5.11) and vdBK14, Cor. 8.14 (a)] with $R=\bar{P}_{0}$, we find that

$$
\begin{aligned}
C_{\bar{P}_{0} \mid P_{0}}(1: \lambda) C(P: Q: \lambda) & =C_{\bar{P}_{0} \mid P}(1: \lambda) C(P: Q: \lambda) \\
& =C_{\bar{P}_{0} \mid Q}(1: \lambda) .
\end{aligned}
$$

Substituting the latter expression in (5.14), we obtain (5.13).

Our next goal is to describe the $C$-function in (5.13) in terms of a standard intertwining operator in case $Q$ and $P_{0}$ are suitably related. For this, we need to introduce additional notation.

Let $\xi \in \widehat{M}_{0}$. We define $C^{\infty}(K: \xi)$ to be the space of functions $f: K \rightarrow \mathscr{H}_{\xi}$ transforming according to the rule

$$
f(m k):=\xi(m) f(k) \quad\left(k \in K, m \in M_{0} \cap K\right) .
$$


Furthermore, we put $\xi_{M}=\left.\xi\right|_{M}$ and define $C^{\infty}\left(K: \xi_{M}\right)$ to be the space of functions $f: K \rightarrow \mathscr{H}_{\xi}$ transforming according to the same rule (5.16) but for $k \in K$ and $m \in M$. Since $M \subseteq M_{0}$, we have a natural inclusion map

$$
i^{\#}: C(K: \xi) \rightarrow C\left(K: \xi_{M}\right) .
$$

Following vdBK14, Sect. 4], we denote by

$$
p^{\#}: C\left(K: \xi_{M}\right) \rightarrow C(K: \xi)
$$

the transpose of this map for the natural sesquilinear pairings coming from the $L^{2}$-inner product on $L^{2}\left(K, \mathscr{H}_{\xi}, d k\right)$.

If $P_{0} \in \mathscr{P}_{\sigma}\left(A_{\mathfrak{q}}\right)$, and $\lambda \in \mathfrak{a}_{\mathfrak{q} C}^{*}$, we denote the realization of the normalized induced representation $\operatorname{Ind}_{P_{0}}^{G}(\xi \otimes \lambda \otimes 1)$ of $G$ in $C^{\infty}(K: \xi)$ according to the compact picture by $\pi_{P_{0}, \xi, \lambda}$. Given a second parabolic subgroup $P_{1} \in \mathscr{P}_{\sigma}\left(A_{\mathfrak{q}}\right)$ we denote by

$$
A\left(P_{1}: P_{0}: \xi: \lambda\right): C^{\infty}(K: \xi) \rightarrow C^{\infty}(K: \xi)
$$

the (meromorphic continuation of) the standard intertwining operator which intertwines the representations $\operatorname{Ind}_{P_{j}}^{G}(\xi \otimes \lambda \otimes 1)$, for $j=0,1$, respectively.

Likewise, if $Q \in \mathscr{P}(A), \xi \in \widehat{M}_{0}$ and $\mu \in \mathfrak{a}_{\mathbb{C}}^{*}$, we denote the realization of the normalized induced representation $\operatorname{Ind}_{Q}^{G}\left(\xi_{M} \otimes \mu \otimes 1\right)$ of $G$ in $C^{\infty}\left(K: \xi_{M}\right)$ according to the compact picture by $\pi_{Q, \xi_{M}, \mu}$.

Given $Q_{1}, Q_{2} \in \mathscr{P}(A)$ we denote by

$$
A\left(Q_{1}: Q_{2}: \xi_{M}: \mu\right): C^{\infty}\left(K: \xi_{M}\right) \rightarrow C^{\infty}\left(K: \xi_{M}\right),
$$

the (meromorphic continuation of) the standard intertwining operator which intertwines the representations

$$
\operatorname{Ind}_{Q_{j}}^{G}\left(\xi_{M} \otimes \mu \otimes 1\right),
$$

for $j=2,1$, respectively.

The two types of parabolically induced representations are related by the maps $i^{\#}$ and $p^{\#}$ defined above. Let $P \in \mathscr{P}(A)$ be a $\mathfrak{q}$-extreme parabolic subgroup, and let $P_{0}$ be the unique parabolic subgroup in $\mathscr{P}_{\sigma}\left(A_{\mathfrak{q}}\right)$ containing $P$. Then $i^{\#}$ intertwines $\pi_{P_{0}, \xi, \lambda}$ with $\pi_{P, \xi_{M}, \lambda-\rho_{P, \mathfrak{h}}}$ and $p^{\#}$ intertwines $\pi_{P, \xi_{M}, \lambda+\rho_{P, \mathfrak{h}}}$ with $\pi_{P_{0}, \xi, \lambda}$, for every $\lambda \in \mathfrak{a}_{\mathfrak{q} \mathbb{C}}^{*}$. We refer to $v$ dBK14, Sect. 4] for further details.

We denote by

$$
\Pi_{\Sigma, \mathbb{R}}\left(\mathfrak{a}_{\mathfrak{q}}^{*}\right)
$$

the set of polynomial functions $\mathfrak{a}_{\mathfrak{q} \mathbb{C}}^{*} \rightarrow \mathbb{C}$ that can be expressed as non-zero products of affine functions of the form $\lambda \mapsto\langle\lambda, \alpha\rangle-c$, where $\alpha \in \Sigma \backslash \mathfrak{a}_{\mathfrak{h}}^{*}$ and $c \in \mathbb{R}$.

Finally, we arrive at the mentioned description of the $C$-function in (5.13).

Proposition 5.5. Let $Q \in \mathscr{P}(A)$, let $P \in \mathscr{P}_{\sigma}(A, Q)$ (see (1.3)), and let $P_{0}$ be the unique minimal $\sigma \theta$-stable parabolic subgroup containing $P$. Then the following assertions are valid.

(a) If $\xi \in \widehat{M}_{0}$ and $T \in C^{\infty}(K: \xi: \tau) \otimes \bar{V}(\xi)$ then, for generic $\lambda \in \mathfrak{a}_{\mathfrak{q} \mathbb{C}}^{*}$,

$$
C_{\bar{P}_{0} \mid Q}(1: \lambda) \psi_{T}=\psi_{\left[p \# \circ A\left(\sigma P: Q: \xi_{M}:-\lambda+\rho_{P, \mathfrak{h}}\right) \circ i \# \otimes I\right] T} .
$$

(b) The function $C_{\bar{P}_{0} \mid Q}(1: \cdot)$ is holomorphic on the set

$$
\left\{\lambda \in \mathfrak{a}_{\mathfrak{q} \mathbb{C}}^{*}: \operatorname{Re}\left\langle-\lambda+\rho_{Q, \mathfrak{h}}, \alpha\right\rangle>0 \text { for all } \alpha \in \Sigma\left(P_{0}\right) \cap \Sigma(Q)\right\} .
$$


(c) Let $B \subseteq \mathfrak{a}_{\mathfrak{q}}^{*}$ be open and bounded. There exists a $p \in \Pi_{\Sigma, \mathbb{R}}\left(\mathfrak{a}_{\mathfrak{q}}^{*}\right)$ such that

$$
\lambda \mapsto p(\lambda) C_{\bar{P}_{0} \mid Q}(1: \lambda)
$$

is holomorphic and of polynomial growth on $B+i \mathfrak{a}_{\mathfrak{q}}^{*}$.

Proof. We first turn to (a). From (5.15) we recall that

$$
C_{\bar{P}_{0} \mid Q}(1: \lambda)=C_{\bar{P}_{0} \mid P_{0}}(1: \lambda) C(P: Q: \lambda) \text {. }
$$

Let $\xi$ and $\psi$ be as in assertion (a). Then it follows from vdBK14, Prop. 8.7] that

$$
C(P: Q: \lambda) \psi_{T}=\psi_{S(\lambda)}
$$

with

$$
S(\lambda)=\left[p^{\#} \circ A\left(Q: P: \xi_{M}:-\lambda+\rho_{P, \mathfrak{h}}\right)^{-1} \circ i^{\#} \otimes I\right] T .
$$

On the other hand, by vdBS97b, Prop. 3.1],

$$
C_{\bar{P}_{0} \mid P_{0}}(1: \lambda) \psi_{S(\lambda)}=\psi_{S^{\prime}(\lambda)},
$$

with

$$
S^{\prime}(\lambda)=\left[A\left(\bar{P}_{0}: P_{0}: \xi:-\lambda\right) \otimes I\right] S(\lambda) .
$$

From (5.20), (5.21) and (5.23) we obtain that

$$
C_{\bar{P}_{0} \mid Q}(1: \lambda) \psi_{T}=\psi_{S^{\prime}(\lambda)}
$$

we will prove (a) by determining $S^{\prime}(\lambda)$.

It follows from vdBK14, Lemma 8.10], that the following diagram commutes, for generic $\lambda \in \mathfrak{a}_{\mathfrak{q} \mathfrak{C}}^{*}$,

$$
\begin{array}{ccc}
C\left(K: \xi_{M}\right) & \stackrel{A\left(\sigma P: P: \xi_{M}:-\lambda+\rho_{P, \mathfrak{h}}\right)}{\longrightarrow} & C\left(K: \xi_{M}\right) \\
p^{\#} \downarrow & & \downarrow p^{\#} \\
C(K: \xi) & A\left(\bar{P}_{0}: P_{0}: \xi:-\lambda\right) & C(K: \xi) .
\end{array}
$$

Taking the commutativity of this diagram into account, we infer by combining (5.22) and (5.24) that

(5.26) $S^{\prime}(\lambda)=\left[p^{\#} \circ A\left(\sigma P: P: \xi_{M}:-\lambda+\rho_{P, \mathfrak{h}}\right) A\left(Q: P: \xi_{M}:-\lambda+\rho_{P, \mathfrak{h}}\right)^{-1} \circ i^{\#} \otimes I\right] T$.

Since $P \succeq Q$, we have $\Sigma(\sigma P) \cap \Sigma(P)=\Sigma(P, \sigma) \subseteq \Sigma(Q) \cap \Sigma(P)$. By application of [KS80, Cor. 7.7] we find that

$$
\begin{aligned}
A(\sigma P & \left.: P: \xi_{M}:-\lambda+\rho_{Q, \mathfrak{h}}\right) \\
& =A\left(\sigma P: Q: \xi_{M}:-\lambda+\rho_{P, \mathfrak{h}}\right) \circ A\left(Q: P: \xi_{M}:-\lambda+\rho_{Q, \mathfrak{h}}\right) .
\end{aligned}
$$

The identity in (5.18) now follows from (5.25), (5.26) and (5.27). Thus, (a) holds.

We turn to (b) and (c). Let $\xi \in \widehat{M}_{0}$ and let $\operatorname{End}\left(C\left(K: \xi_{M}\right)\right)$ denote the space of bounded linear endomorphisms of the Banach space $C\left(K: \xi_{M}\right)$. Then as a $\operatorname{End}\left(C\left(K: \xi_{M}\right)\right)$-valued function, the standard intertwiner $A\left(\sigma P: Q: \xi_{M}: \mu\right)$ depends holomorphically on $\mu \in \mathfrak{a}_{\mathbb{C}}^{*}$ satisfying

$$
\operatorname{Re}\langle\mu, \alpha\rangle>0, \quad(\alpha \in \Sigma(\sigma \bar{P}) \cap \Sigma(Q)) .
$$

Indeed, this is a straightforward consequence of the convergence of the integral defining the intertwining operator, asserted in KS80, Thm. 4.2].

Since $\Sigma\left(P_{0}\right)=\Sigma(P) \backslash \mathfrak{a}_{\mathfrak{h}}^{*}$, we have $\Sigma\left(P_{0}\right) \cap \Sigma(Q) \subseteq \Sigma(\sigma \bar{P}) \cap \Sigma(Q)$. Thus, if $\lambda \in \mathfrak{a}_{\mathfrak{q} \mathbb{C}}^{*}$ belongs to the set (5.19), then $\mu=-\lambda+\rho_{P, \mathfrak{h}}$ satisfies (5.28). We infer that 
$A\left(\sigma P: Q: \xi_{M}:-\lambda+\rho_{P, \mathfrak{h}}\right)$ is a holomorphic $\operatorname{End}\left(C\left(K: \xi_{M}\right)\right)$-valued function of $\lambda$ in the set (5.19). In view of (5.18) we now infer (b).

Finally, we turn to (c). Let $\xi \in \widehat{M}_{0}$ and let $\mathscr{A}_{M, 2}(\tau)_{\xi}$ denote the image of $C(K: \xi \tau) \otimes \bar{V}(\xi)$ under the map (5.8). We may select a finite set of $K$-types $\vartheta \subseteq \widehat{K}$ such that $C(K: \xi: \tau) \subseteq C(K: \xi)_{\vartheta} \otimes V_{\tau}$. In view of vdBS12, Thm. 1.5] there exists a polynomial function $q_{\xi}: \mathfrak{a}_{\mathbb{C}}^{*} \rightarrow \mathbb{C}$ which is a product of linear factors of the form $\mu \mapsto\langle\mu, \alpha\rangle-c$, with $\alpha \in \Sigma(\sigma \bar{P}) \cap \Sigma(Q)$ and $c \in \mathbb{R}$ such that

$$
\left.\mu \mapsto q_{\xi}(\mu) A\left(\sigma P: Q: \xi_{M}: \mu\right)\right|_{C\left(K: \xi_{M}\right)_{\vartheta}}
$$

is holomorphic and polynomially bounded on the set $-B+\rho_{Q, \mathfrak{h}}+i \mathfrak{a}^{*}$. It follows that the function of $\lambda \in \mathfrak{a}_{\mathfrak{q} \mathbb{C}}^{*}$ arising from (5.29) by the substitution $\mu=-\lambda+\rho_{Q, \mathfrak{h}}$ is holomorphic and polynomially bounded on $B+i \mathfrak{a}_{\mathfrak{q}}^{*}$. Define

$$
p_{\xi}(\lambda):=q_{\xi}\left(-\lambda+\rho_{Q, \mathfrak{h}}\right) .
$$

Then $p_{\xi} \in \Pi_{\Sigma, \mathbb{R}}\left(\mathfrak{a}_{\mathfrak{q}}^{*}\right)$ because $\Sigma(\sigma \bar{P}) \cap \Sigma(Q) \subseteq \Sigma \backslash \mathfrak{a}_{\mathfrak{h}}^{*}$, and in view of (5.18) it follows that

$$
\lambda \mapsto p_{\xi}(\lambda) C_{\bar{P}_{0} \mid Q}(1: \lambda)_{\mathscr{A}_{M, 2}(\tau)_{\xi}}
$$

is holomorphic and polynomially bounded on $B+i \mathfrak{a}_{\mathfrak{q}}^{*}$. The result now follows by finiteness of the sum (5.8).

5.3. The $\tau$-spherical Fourier transform. Let $Q \in \mathscr{P}(A)$ and let $\left(\tau, V_{\tau}\right)$ be a finite dimensional unitary representation of $K$. For $\phi \in C_{c}^{\infty}(G / H: \tau)$, we define the $\tau$-spherical Fourier transform $\mathfrak{F}_{Q, \tau} \phi$ to be the meromorphic function $\mathfrak{a}_{\mathfrak{q} \mathbb{C}}^{*} \rightarrow \mathscr{A}_{M, 2}(\tau)$ determined by

$$
\left\langle\mathfrak{F}_{Q, \tau} \phi(\lambda), \psi\right\rangle=\int_{G / H}\langle\phi(x), E(Q: \psi:-\bar{\lambda})(x)\rangle_{\tau} d x
$$

for $\psi \in \mathscr{A}_{M, 2}(\tau)$ and generic $\lambda \in \mathfrak{a}_{\mathfrak{q C}}^{*}$.

Proposition 5.6. Let $\phi \in C_{c}^{\infty}(G / H: \tau)$. Then $\mathfrak{F}_{Q, \tau} \phi$ is holomorphic on an open neighborhood of $-\widehat{\Upsilon}_{Q}$; see (5.3).

Proof. This follows directly from vdBK14, Cor. 8.5].

Before proceeding we will first discuss how this Fourier transform is related to the $\tau$-spherical Fourier transform $\mathcal{F}_{\bar{P}_{0}} \phi$ defined in vdBS97b, Eqn. (59)], for $P_{0}$, hence $\bar{P}_{0}$, a minimal $\sigma \theta$-stable parabolic subgroup from $\mathscr{P}_{\sigma}\left(A_{\mathfrak{q}}\right)$. The last mentioned transform is defined to be the meromorphic function $\mathfrak{a}_{\mathfrak{q C}}^{*} \rightarrow \mathscr{A}_{M, 2}(\tau)$ given by

$$
\left\langle\mathcal{F}_{\bar{P}_{0}} \phi(\lambda), \psi\right\rangle=\int_{G / H}\left\langle f(x), E^{\circ}\left(\bar{P}_{0}: \psi:-\bar{\lambda}\right)(x)\right\rangle_{\tau} d x
$$

for $\psi \in \mathscr{A}_{M, 2}(\tau)$ and generic $\lambda \in \mathfrak{a}_{\mathfrak{q C}}^{*}$

Proposition 5.7. Let $P_{0} \in \mathscr{P}_{\sigma}\left(A_{\mathfrak{q}}\right)$ and $\phi \in C_{c}^{\infty}(G / H: \tau)$. Then

$$
\mathfrak{F}_{Q, \tau} \phi(\lambda)=C_{\bar{P}_{0} \mid Q}(1:-\bar{\lambda})^{*} \mathcal{F}_{\bar{P}_{0}} \phi(\lambda) \text {. }
$$

for generic $\lambda \in \mathfrak{a}_{\mathfrak{q} \mathbb{C}}^{*}$.

Proof. The identity follows directly from Lemma 5.4.

Given $R>0$ we write $B_{R}$ for the open ball in $\mathfrak{a}_{\mathfrak{q}}$ with center 0 and radius $R$. Furthermore, we define

$$
C_{R}^{\infty}(G / H: \tau):=\left\{\phi \in C_{c}^{\infty}(G / H: \tau): \operatorname{supp} \phi \subseteq K \exp \left(B_{R}\right) \cdot H\right\} .
$$


Proposition 5.8. Let $B \subseteq \mathfrak{a}_{\mathfrak{q}}^{*}$ be open and bounded. There exists a $p \in \Pi_{\Sigma, \mathbb{R}}\left(\mathfrak{a}_{\mathfrak{q}}^{*}\right)$ such that $\lambda \mapsto p(-\lambda) E(Q: \psi:-\lambda)$ is holomorphic on $B+i \mathfrak{a}_{\mathfrak{q}}^{*}$ for every $\psi \in$ $\mathscr{A}_{M, 2}(\tau)$. Moreover, if $\phi \in C_{c}^{\infty}(G / H, \tau)$, then

$$
\lambda \mapsto p(\lambda) \mathfrak{F}_{Q, \tau} \phi(\lambda)
$$

is holomorphic on $B+i \mathfrak{a}_{\mathfrak{q}}^{*}$.

Let $R>0$. There exist a constant $C_{R}>0$ and for every $N \in \mathbb{N}$ a continuous seminorm $\nu_{N}$ on $C_{R}^{\infty}(G / H: \tau)$ such that

$$
\left\|p(\lambda) \mathfrak{F}_{Q, \tau} \phi(\lambda)\right\| \leq(1+\|\lambda\|)^{-N} e^{C_{R}\|\operatorname{Re} \lambda\|} \nu_{N}(\phi)
$$

for every $\phi \in C_{R}^{\infty}(G / H: \tau)$ and all $\lambda \in B+i \mathfrak{a}_{\mathfrak{q}}^{*}$.

Proof. We fix a q-extreme parabolic subgroup $P \in \mathscr{P}(A)$ such that $P \succeq Q$. Let $P_{0}$ be the unique subgroup in $\mathscr{P}_{\sigma}\left(A_{\mathfrak{q}}\right)$ such that $P_{0} \supseteq P$. By vdBS99, Prop. 3.1] there exists a $p_{1} \in \Pi_{\Sigma, \mathbb{R}}\left(\mathfrak{a}_{\mathfrak{q}}^{*}\right)$ such that

$$
\lambda \mapsto p_{1}(-\lambda) E^{\circ}\left(\bar{P}_{0}: \psi:-\lambda\right)
$$

is holomorphic on $B+i \mathfrak{a}_{\mathfrak{q}}^{*}$ for every $\psi \in \mathscr{A}_{M, 2}(\tau)$. This implies that $p_{1} \mathcal{F}_{\bar{P}_{0}} \phi$ is holomorphic on $B+i \mathfrak{a}_{\mathfrak{q}}^{*}$ for every $\phi \in C_{c}^{\infty}(G / H: \tau)$. Furthermore, by vdBS99, Lemma 4.4] there exist a constant $C_{R}>0$ and for every $N \in \mathbb{N}$ a continuous seminorm $\nu_{N}$ on $C_{R}^{\infty}(G / H: \tau)$ such that

$$
\left\|p_{1}(\lambda) \mathcal{F}_{\bar{P}_{0}, \tau} \phi(\lambda)\right\| \leq(1+\|\lambda\|)^{-N} e^{C_{R}\|\operatorname{Re} \lambda\|} \nu_{N}(\phi)
$$

for every $\phi \in C_{R}^{\infty}(G / H: \tau)$ and all $\lambda \in B+i \mathfrak{a}_{\mathfrak{q}}^{*}$.

Choose $p_{2} \in \Pi_{\Sigma, \mathbb{R}}\left(\mathfrak{a}_{\mathfrak{q}}^{*}\right)$ as in Proposition $5.5(\mathrm{~b}, \mathrm{c})$ and put $p=p_{1} p_{2}$. Then the result follows in view of Proposition [5.7, by combining the above assertions with those of Proposition 5.5(b,c).

\section{The $\tau$-SPherical Harish-Chandra transform}

6.1. Definition and relation with the spherical Fourier transform. We assume that $Q \in \mathscr{P}(A)$ and that $\left(\tau, V_{\tau}\right)$ is a finite dimensional unitary representation of $K$. Recall the definition of the character $\delta_{Q}$ on $L$ by (2.2); see also (2.3). The following definition makes use of the notation (5.1).

Definition 6.1. For a function $\phi \in C_{c}^{\infty}(G / H: \tau)$ we define its $\tau$-spherical HarishChandra transform $\mathcal{H}_{Q, \tau} \phi$ to be the function $A_{\mathfrak{q}} \rightarrow \mathscr{A}_{M, 2}(\tau)$ given by

$$
\left(\mathcal{H}_{Q, \tau} \phi(a)\right)_{v}(m):=\delta_{Q}(a) \int_{N_{Q^{v}} / H_{N_{Q^{v}}}} \phi(\text { mavn }) d n
$$

for $v \in \mathscr{W}, m \in M$ and $a \in A_{\mathfrak{q}}$.

It is easily seen that $\mathcal{H}_{Q, \tau}$ defines a continuous linear map $C_{c}^{\infty}(G / H: \tau) \rightarrow$ $C^{\infty}\left(A_{\mathfrak{q}}\right) \otimes \mathscr{A}_{M, 2}(\tau)$. The $\tau$-spherical Harish-Chandra transform $\mathcal{H}_{Q, \tau}$ is related to the Harish-Chandra transform introduced in Definition 2.12. Namely, the following result is valid.

Lemma 6.2. Let $\phi \in C_{c}^{\infty}(G / H: \tau)$. Then for $a \in A_{\mathfrak{q}}$ and $\psi \in \mathscr{A}_{M, 2}(\tau)$,

$$
\left\langle\mathcal{H}_{Q, \tau} \phi(a), \psi\right\rangle=\sum_{v \in \mathscr{W}} \mathcal{H}_{Q^{v}}\left(\left\langle\phi(\cdot), \tau\left(v^{-1}\right) \psi_{v}(e)\right\rangle_{\tau}\right)\left(v^{-1} a v\right) .
$$


Proof. Let $\phi \in C_{c}^{\infty}(G / H: \tau), \psi \in \mathscr{A}_{M, 2}(\tau)$ and $a \in A_{\mathfrak{q}}$. Recall that ${ }^{v} H$ denotes $v H v^{-1}$ for $v \in \mathscr{W}$. Then

$$
\begin{aligned}
& \left\langle\mathcal{H}_{Q, \tau} \phi(a), \psi\right\rangle=\sum_{v \in \mathscr{W}} \int_{M /\left(M \cap^{v} H\right)}\left\langle\left(\mathcal{H}_{Q, \tau} \phi(a)\right)_{v}(m), \psi_{v}(m)\right\rangle_{\tau} \\
& =\sum_{v \in \mathscr{W}} \int_{M /\left(M \cap^{v} H\right)} \delta_{Q}(a) \int_{N_{Q^{v}} / H_{N_{Q^{v}}}}\left\langle\phi(m a v n), \psi_{v}(m)\right\rangle_{\tau} \\
& =\sum_{v \in \mathscr{W}} \int_{M /\left(M \cap^{v} H\right)} \delta_{Q^{v}}\left(v^{-1} a v\right) \int_{N_{Q^{v}} / H_{N_{Q^{v}}}}\left\langle\phi\left(m v v^{-1} a v n\right), \psi_{v}(m)\right\rangle_{\tau} .
\end{aligned}
$$

We now use that $\tau$ is unitary and that the measure on $M /\left(M \cap{ }^{v} H\right)$ is normalized, and thus we conclude that the last expression is equal to

$$
\sum_{v \in \mathscr{W}} \delta_{Q^{v}}\left(v^{-1} a v\right) \int_{N_{Q^{v}} / H_{N_{Q^{v}}}}\left\langle\phi\left(v^{-1} a v n\right), \tau\left(v^{-1}\right) \psi_{v}(e)\right\rangle_{\tau} .
$$

Finally, the claim follows from the definition of the Harish-Chandra transform (Definition 2.12).

\section{Corollary 6.3.}

(a) Let $P \in \mathscr{P}_{\sigma}(A, Q)$. Then the spherical Harish-Chandra transform $\mathcal{H}_{Q, \tau}$ is a continuous linear map $C_{c}^{\infty}(G / H: \tau) \rightarrow L^{1}\left(A_{\mathfrak{q}}, \delta_{P}^{-1} d a\right) \otimes \mathscr{A}_{M, 2}(\tau)$.

(b) Let $\phi \in C_{c}^{\infty}(G / H: \tau)$ be supported in $K \exp C \cdot H$, with $C \subseteq \mathfrak{a}_{\mathfrak{q}}$ compact, convex and invariant under the action of $N_{K \cap H}\left(\mathfrak{a}_{\mathfrak{q}}\right)$. Then

$$
\operatorname{supp} \mathcal{H}_{Q, \tau}(\phi) \subseteq \bigcup_{v \in \mathscr{W}} \exp \left(C+v \Gamma\left(Q^{v}\right)\right)
$$

Proof. It follows from Proposition 3.9 that $\delta_{P^{v}}^{-1}\left(\mathcal{H}_{Q^{v}} \otimes I\right)$ defines a continuous linear map $C_{c}^{\infty}(G / H: \tau) \rightarrow L^{1}\left(L / H_{L}: \tau_{M}\right)$. Since $\left.L / H_{L} \simeq M / M \cap H \times A_{\mathfrak{q}}\right)$, it follows that restriction to $A_{\mathfrak{q}}$ defines a continuous linear map $L^{1}\left(L / H_{L}: \tau_{M}\right) \rightarrow A_{\mathfrak{q}} \otimes V_{\tau}$. In view of Lemma 6.2 assertion (a) of the corollary now follows.

For (b), assume that $\phi \in C_{c}^{\infty}(G / H: \tau)$ has a support as stated. Then by Proposition 3.8 the support of $\left.\left(\mathcal{H}_{Q^{v}} \otimes I\right)(\phi)\right|_{A_{\mathfrak{q}}}$ is contained in $\exp \left(C+\Gamma\left(Q^{v}\right)\right)$. In view of the $N_{K \cap H}\left(\mathfrak{a}_{\mathfrak{q}}\right)$-invariance of $C$, the inclusion (6.3) now follows by application of Lemma 6.2.

It follows from this corollary that for $\phi \in C_{c}^{\infty}(G / H: \tau)$ the Euclidean Fourier transform

$$
\mathcal{F}_{A_{\mathfrak{q}}}\left(\mathcal{H}_{Q, \tau} \phi\right)(\lambda)=\int_{A_{\mathfrak{q}}} \mathcal{H}_{Q, \tau} \phi(a) a^{-\lambda} d a,
$$

is well-defined for $\lambda$ in the subset $-\Upsilon_{Q} \subseteq \mathfrak{a}_{\mathfrak{q} C}^{*}$, with absolutely convergent integral, and defines a holomorphic $\mathscr{A}_{M, 2}(\tau)$-valued function on the interior of this set.

Proposition 6.4. Let $\phi \in C_{c}^{\infty}(G / H: \tau)$. Then for $\lambda \in-\Upsilon_{Q}$,

$$
\mathfrak{F}_{Q, \tau} \phi(\lambda)=\mathcal{F}_{A_{\mathfrak{q}}}\left(\mathcal{H}_{Q, \tau} \phi\right)(\lambda) .
$$

Before turning to the proof of this result, we first prove a lemma. 
Lemma 6.5. Let $\omega \in \mathscr{D}_{\mathfrak{g} / \mathfrak{h}_{Q}}$. Let $\psi \in C\left(G: H_{Q}: \Delta_{G / H_{Q}}\right)$ and assume that the associated density $\psi_{\omega} \in \mathscr{D}_{G / H_{Q}}$ is integrable. Then

$$
\int_{G / H_{Q}} \psi_{\omega}=\int_{K} \int_{A_{\mathfrak{q}}} \int_{N_{Q} / H_{N_{Q}}} a^{2 \rho_{Q}} \psi(k a n) d n d a d k
$$

up to a positive constant which only depends on the normalization of the measures and the densities.

Proof. In this proof we will need to introduce several densities. For each quotient $S / T$ of a Lie group $S$ by a closed subgroup $T$ that appears below, we choose a positive density $\omega_{S / T} \in \mathscr{D}_{\mathfrak{s} / \mathfrak{t}}$. We leave it to the reader to check that these densities may be normalized in such a manner that the stated equalities are valid.

By Theorem 5.1,

$$
\int_{G / H_{Q}} \psi_{\omega}=\int_{G / Q} I_{g}(\psi) d l_{g}([e])^{-1 *} \omega_{G / Q},
$$

where

$$
I_{g}(\psi)=\int_{Q / H_{Q}} \psi(g q) \Delta_{G / Q}(q) d l_{q}([e])^{-1 *} \omega_{Q / H_{Q}} .
$$

Since the canonical map $\zeta: K / M \rightarrow G / Q$ is a $K$-equivariant diffeomorphism we may rewrite the integral on the right-hand side of (6.4) as an integral over $K / M$ of the pull-back density

$$
\zeta^{*}\left(g \mapsto I_{g}(\psi) d l_{g}([e])^{-1 *} \omega_{G / Q}\right)_{k}=I_{k}(\psi) d l_{k}(e)^{-1 *} \omega_{K / M} .
$$

Now $k \mapsto I_{k}(\psi)$ is right $M$-invariant, and $k \mapsto d l_{k}(e)^{-1 *} \omega_{K / M}$ defines a left $K$ equivariant density on $K / M$. Hence,

$$
\int_{G / Q} \psi_{\omega}=\int_{K} I_{k}(\psi) d k
$$

Next, we fix $k \in K$. Applying Theorem 5.1 to the integral for $I_{k}(\psi)$, given by (6.5) with $g=k$, we infer that

$$
I_{k}(\psi)=\int_{Q / H_{L} N_{Q}} J_{y}\left(l_{k}^{*} \psi \Delta_{G / Q}\right) d l_{y}([e])^{-1 *} \omega_{Q / H_{L} N_{Q}},
$$

where

$$
\begin{aligned}
J_{y}\left(l_{k}^{*} \psi \Delta_{G / Q}\right) & =\int_{H_{L} N_{Q} / H_{Q}} \psi(k y x) \Delta_{G / Q}(y x) \Delta_{Q / H_{L} N_{Q}}(x) d l_{x}([e])^{-1 *} \omega_{H_{L} N_{Q} / H_{Q}} \\
(6.8) & =\int_{H_{L} N_{Q} / H_{Q}} \psi(k y x) \Delta_{G / Q}(y x) d l_{x}([e])^{-1 *} \omega_{H_{L} N_{Q} / H_{Q}} .
\end{aligned}
$$

In the latter equality we have used that $\Delta_{Q / H_{L} N_{Q}}=1$. Indeed, by nilpotency of $N_{Q}$ it is evident that $\left.\Delta_{Q / H_{L} N_{Q}}\right|_{N_{Q}}=1$. On the other hand, $\left.\Delta_{Q / H_{L}{ }_{Q}}\right|_{H_{L}}=\Delta_{L / H_{L}}=1$ by unimodularity of $L$ and $H_{L}$.

To complete the proof we will rewrite both integrals (6.7) and (6.8), respectively. Starting with the first, we note that the map

$$
\eta: A_{\mathfrak{q}} \times M / H_{M} \rightarrow Q / H_{L} N_{Q} ; \quad(a, m) \mapsto a m H_{L} N_{Q}
$$


is a $A_{\mathfrak{q}} \times M$-equivariant diffeomorphism. Hence, $\eta^{*}\left(y \mapsto d l_{y}([e])^{-1 *} \omega_{Q / L_{H} N_{Q}}\right)$ is a left $A_{\mathfrak{q}} \times M$-invariant density. Accordingly, the integral (6.7) may be rewritten as

$$
\begin{aligned}
I_{k}(\psi) & =\int_{M / H_{M}} \int_{A_{\mathfrak{q}}} J_{a m}\left(l_{k}^{*} \psi \Delta_{G / Q}\right) d a d \bar{m} \\
& =\int_{M} \int_{A_{\mathfrak{q}}} J_{m a}\left(l_{k}^{*} \psi \Delta_{G / Q}\right) d a d m .
\end{aligned}
$$

Likewise, the map

$$
\vartheta: N_{Q} / H_{N_{Q}} \rightarrow H_{L} N_{Q} / H_{Q} ; \quad n H_{N_{Q}} \mapsto n H_{Q}
$$

is a left $N_{Q}$-equivariant diffeomorphism. Therefore, $\vartheta^{*}\left(x \mapsto d l_{x}([e])^{-1 *} \omega_{H_{L} N_{Q} / H_{Q}}\right)$ is an $N_{Q}$-invariant density on $N_{Q} / H_{N_{Q}}$. Accordingly, we find that (6.8) may be rewritten as

$$
J_{y}\left(l_{k}^{*} \psi \Delta_{G / Q}\right)=\int_{N_{Q} / H_{N_{Q}}} \psi(k y n) \Delta_{G / Q}(y n) d n .
$$

Combining (6.6), (6.9) and (6.10), we obtain that

$$
\begin{aligned}
\int_{G / H_{Q}} \psi_{\omega} & =\int_{K} \int_{M} \int_{A_{\mathfrak{q}}} \int_{N_{Q} / H_{N_{Q}}} \Delta_{G / Q}(\operatorname{man}) \psi(k \operatorname{man}) d n d a d m d k \\
& =\int_{K} \int_{A_{\mathfrak{q}}} \int_{N_{Q} / H_{N_{Q}}} a^{2 \rho_{Q}} \psi(k a n) d n d a d k .
\end{aligned}
$$

Proof of Proposition 6.4. For each $v \in \mathscr{W}$ let $\omega_{H / H_{Q^{v}}} \in \mathscr{D}_{\mathfrak{h} / \mathfrak{h}_{Q^{v}}}$ be as in (5.2). Let $\phi \in C_{c}^{\infty}(G / H: \tau)$ and $\psi \in \mathscr{A}_{M, 2}(\tau)$. Then for $\lambda \in-\Upsilon_{Q}$,

$$
\begin{aligned}
& \left\langle\mathfrak{F}_{Q, \tau} \phi(\lambda), \psi\right\rangle=\int_{G / H}\langle\phi(x), E(Q: \psi:-\bar{\lambda})(x)\rangle_{\tau} d x \\
& =\sum_{v \in \mathscr{W}} \int_{G / H}\left\langle\phi(x), E_{v_{H}}\left(Q: \psi_{v}:-\bar{\lambda}\right)\left(x v^{-1}\right)\right\rangle_{\tau} d x \\
& =\sum_{v \in \mathscr{W}} \int_{G / H}\left(\int_{H / H_{Q^{v}}}\left\langle\phi(x), \psi_{v, Q,-\bar{\lambda}}\left(x h v^{-1}\right)\right\rangle_{\tau} d l_{h}(e)^{*-1} \omega_{H / H_{Q^{v}}}\right) d l_{x}(e)^{*-1} \omega_{G / H} .
\end{aligned}
$$

Here $\psi_{v, Q,-\bar{\lambda}}$ is defined as in (5.4). We now apply Theorem 5.1 to the term for $v$ in order to rewrite the repeated integral as a single integral over $G / H_{Q^{v}}$ and obtain

$$
\left\langle\mathfrak{F}_{Q, \tau} \phi(\lambda), \psi\right\rangle=\sum_{v \in \mathscr{W}} \int_{G / H_{Q^{v}}}\left\langle\phi(y), \psi_{v, Q,-\bar{\lambda}}\left(y v^{-1}\right)\right\rangle_{\tau} d l_{y}(e)^{*-1} \omega_{G_{H} H_{Q^{v}}} .
$$

By Lemma 6.5 this expression is equal to

$$
\sum_{v \in \mathscr{W}} \int_{K} \int_{A_{q}} \int_{N_{Q^{v}} / H_{N_{Q^{v}}}} a^{2 \rho_{Q^{v}}}\left\langle\phi(k a n), \psi_{v, Q,-\bar{\lambda}}\left(k a n v^{-1}\right)\right\rangle_{\tau} d n d a d k
$$

By $\tau$-sphericality and unitarity of $\tau$ it follows that each integrand is independent of $k$. Furthermore, by our chosen normalization of Haar measure, $d k(K)=1$ so that the integral over $K$ can be removed. By substituting $a^{v}:=v^{-1} a v$ for $a$ and using 
the right $A N_{Q^{-}}$equivariance of $\psi_{Q, v,-\bar{\lambda}}$, we thus find

$$
\begin{aligned}
\left\langle\mathfrak{F}_{Q, \tau} \phi(\lambda), \psi\right\rangle & =\sum_{v \in \mathscr{W}} \int_{A_{\mathfrak{q}}} \int_{N_{Q^{v}} / H_{N_{Q} v}} a^{2 \rho_{Q}}\left\langle\phi\left(a^{v} n\right), \tau(v)^{-1} \psi_{v, Q,-\bar{\lambda}}(a)\right\rangle_{\tau} d n d a d k \\
& =\sum_{v \in \mathscr{W}} \int_{A_{\mathfrak{q}}} \int_{N_{Q^{v}} / H_{N_{Q^{v}}}} a^{-\lambda+\rho_{Q}-\rho_{Q, \mathfrak{h}}}\left\langle\phi\left(a^{v} n\right), \tau(v)^{-1} \psi_{v}(e)\right\rangle_{\tau} d n d a d k \\
& =\int_{A_{\mathfrak{q}}} a^{-\lambda} \sum_{v \in \mathscr{W}} \mathcal{H}_{Q^{v}}\left(\left\langle\phi(\cdot), \tau(v)^{-1} \psi(e)\right\rangle_{\tau}\right)\left(a^{v}\right) d a .
\end{aligned}
$$

Using Lemma 6.2 we finally obtain

$$
\left\langle\mathfrak{F}_{Q, \tau} \phi(\lambda), \psi\right\rangle=\int_{A_{\mathfrak{q}}} a^{-\lambda}\left\langle\mathcal{H}_{Q, \tau}(\phi)(a), \psi\right\rangle d a .
$$

Since $\psi$ was arbitrary, the result follows.

6.2. Invariant differential operators. In this section we assume that $P_{0}$ is a parabolic subgroup from $\mathscr{P}_{\sigma}\left(A_{\mathfrak{q}}\right)$ and write $P_{0}=M_{0} A_{0} N_{0}$ for its Langlands decomposition; then $A_{0} \subseteq A$ and $\mathfrak{a}_{\mathfrak{q}}=\mathfrak{a}_{0} \cap \mathfrak{q}$. Furthermore, $M_{0} / M_{0} \cap H=M / M \cap H$ as homogeneous spaces for $M$; see vdBK14, Lemma 4.3]. Accordingly,

$$
\mathfrak{g}=\mathfrak{n}_{0} \oplus(\mathfrak{l}+\mathfrak{h}) ;
$$

where $\mathfrak{l}=\mathfrak{m} \oplus \mathfrak{a}$ is the Lie algebra of $L=M A$. Let $\mathbb{D}(G / H)$ be the algebra of invariant differential operators on $G / H$. Then the right-regular representation of $G$ on $C^{\infty}(G)$ induces an isomorphism

$$
r: U(\mathfrak{g})^{H} /\left(U(\mathfrak{g})^{H} \cap U(\mathfrak{g}) \mathfrak{h}\right) \stackrel{\simeq}{\longrightarrow} \mathbb{D}(G / H) ;
$$

see $\mathrm{vdB92}$, Sect. 2] for details. Let

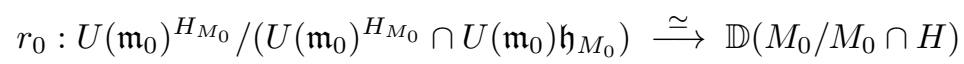

be the analogous isomorphism onto the algebra of left $M_{0}$-invariant differential operators on $M_{0} / M_{0} \cap H$. Let $\mathbb{D}\left(A_{\mathfrak{q}}\right)$ denote the algebra of bi-invariant differential operators on $A_{\mathfrak{q}}$. Then the right regular representation induces an algebra isomorphism $U\left(\mathfrak{a}_{\mathfrak{q}}\right)=S\left(\mathfrak{a}_{\mathfrak{q}}\right) \simeq \mathbb{D}\left(A_{\mathfrak{q}}\right)$. We define the canonical algebra embedding $\mu: \mathbb{D}(G / H) \hookrightarrow \mathbb{D}\left(M_{0} / M_{0} \cap H\right) \otimes \mathbb{D}\left(A_{\mathfrak{q}}\right)$ as in vdB92, Sect. 2]. It is independent of the choice of parabolic subgroup $P_{0}$. We will give a suitable description of $\mu$ in terms of $P_{0}$, which is somewhat different from the one in vdB92.

To prepare for this, let $\mathfrak{m}_{0 n}$ be the ideal of $\mathfrak{m}_{0}$ generated by $\mathfrak{m}_{0} \cap \mathfrak{a}$ and let $M_{0 n}$ be the corresponding analytic subgroup of $M_{0}$. Then $M_{0}=M M_{0 n}$ and $M_{0 n}$ acts trivially on $M_{0} / M_{0} \cap H$; see vdBK14, Lemma 4.3]. Therefore, the inclusion $M \rightarrow M_{0}$ induces a natural isomorphism

$$
\mathbb{D}\left(M_{0} / M_{0} \cap H\right) \simeq \mathbb{D}(M / M \cap H),
$$

via which we shall identify their elements. As before, the right regular representation induces an isomorphism $U(\mathfrak{m})^{H_{M}} / U(\mathfrak{m})^{H_{M}} \cap U(\mathfrak{m}) \mathfrak{h}_{M} \simeq \mathbb{D}(M / M \cap H)$. Furthermore, since $\mathfrak{m}_{0 n} \subseteq \mathfrak{h}$, the inclusion $\mathfrak{m} \hookrightarrow \mathfrak{m}_{0}$ induces an isomorphism

$$
U(\mathfrak{m})^{H_{M}} / U(\mathfrak{m})^{H_{M}} \cap U(\mathfrak{m}) \mathfrak{h}_{M} \simeq U\left(\mathfrak{m}_{0}\right)^{H_{M_{0}}} /\left(U\left(\mathfrak{m}_{0}\right)^{H_{M_{0}}} \cap U\left(\mathfrak{m}_{0}\right) \mathfrak{h}_{M_{0}}\right)
$$

which is compatible with $r_{0}$ and the identification (6.13). Accordingly, we may view $\mu$ as an algebra embedding

$$
\mu: \mathbb{D}(G / H) \hookrightarrow \mathbb{D}(M / M \cap H) \otimes \mathbb{D}\left(A_{\mathfrak{q}}\right) .
$$


Remark 6.6. In the formulation of the following result, we will write $e^{ \pm \rho_{P_{0}}}$ for the continuous linear endomorphism of $C^{\infty}\left((M / M \cap H) \times A_{\mathfrak{q}}\right)$ given by multiplication with the similarly denoted function $e^{ \pm \rho_{P_{0}}}:(m, a) \mapsto a^{ \pm \rho_{P_{0}}}$.

Lemma 6.7. Let $D \in \mathbb{D}(G / H)$ and let $D_{0} \in \mathbb{D}(M / M \cap H) \otimes \mathbb{D}\left(A_{\mathfrak{q}}\right)$ be the element

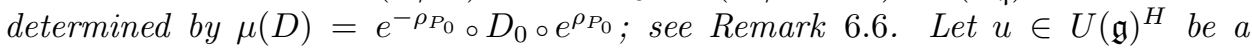
representative of $D$ and let $u_{0} \in U(\mathfrak{m})^{H_{M}} \otimes \mathbb{D}\left(A_{\mathfrak{q}}\right)$ be a representative of $D_{0}$. Then

(a) $u-u_{0} \in \mathfrak{n}_{P_{0}} U(\mathfrak{g}) \oplus U(\mathfrak{g}) \mathfrak{h}$.

(b) Furthermore, if $Q \in \mathscr{P}(A)$ satisfies $\Sigma(Q, \sigma \theta) \subseteq \Sigma\left(P_{0}\right)$, then

$$
u-u_{0} \in\left(\mathfrak{n}_{P_{0}} \cap \mathfrak{n}_{Q}\right) U(\mathfrak{g})+U(\mathfrak{g}) \mathfrak{h} .
$$

Proof. We start with (a). Note that $U(\mathfrak{m})^{H_{M}} \subseteq U\left(\mathfrak{m}_{0}\right)^{H_{M_{0}}}+U\left(\mathfrak{m}_{0}\right) \mathfrak{h}_{M_{0}}$. Thus, if $v_{0}$ is a representative for $D_{0}$ in $U\left(\mathfrak{m}_{0}\right)^{H_{M_{0}}} \otimes \mathbb{D}\left(A_{\mathfrak{q}}\right)$, then $u_{0}-v_{0} \in U(\mathfrak{g}) \mathfrak{h}$ and it suffices to prove the assertion (a) with $v_{0}$ in place of $u_{0}$. The resulting assertion immediately follows from the definition of $\mu$ in $\mathrm{vdB92}$, Sect. 2].

We turn to (b). In view of (6.11) and the PBW theorem, it suffices to show that the image $u_{1}$ of $u-u_{0}$ in $\mathfrak{n}_{P_{0}} U\left(\mathfrak{n}_{P_{0}}\right) \otimes U(\mathfrak{l}) / U(\mathfrak{l}) \mathfrak{h}_{L}$ in fact belongs to the subspace $\left(\mathfrak{n}_{P_{0}} \cap \mathfrak{n}_{Q}\right) U\left(\mathfrak{n}_{P_{0}}\right) \otimes U(\mathfrak{l}) / U(\mathfrak{l}) \mathfrak{h}_{L}$. The element $u_{1}$ is invariant under $\operatorname{ad}\left(\mathfrak{a}_{\mathfrak{h}}\right)$, as both $u$ and $u_{0}$ are. Since $\mathfrak{a}_{\mathfrak{h}}$ centralizes $\mathfrak{l}$, we have

$$
u_{1} \in\left[\mathfrak{n}_{P_{0}} U\left(\mathfrak{n}_{P_{0}}\right)\right]^{\mathfrak{a}_{\mathfrak{h}}} \otimes U(\mathfrak{l}) / U(\mathfrak{l}) \mathfrak{h}_{L} .
$$

By the PBW theorem we have the following direct sum decomposition into $\operatorname{ad}\left(\mathfrak{a}_{\mathfrak{h}}\right)$ invariant subspaces:

$$
\mathfrak{n}_{P_{0}} U\left(\mathfrak{n}_{P_{0}}\right)=\left(\mathfrak{n}_{P_{0}} \cap \mathfrak{n}_{Q}\right) U\left(\mathfrak{n}_{P_{0}}\right) \oplus\left(\mathfrak{n}_{P_{0}} \cap \overline{\mathfrak{n}}_{Q}\right) U\left(\mathfrak{n}_{P_{0}} \cap \overline{\mathfrak{n}}_{Q}\right) .
$$

The $\mathfrak{a}_{\mathfrak{h}}$-weights of the second summand are all of the form $\mu=\alpha_{1}+\cdots+\alpha_{k}$, with $k \geq 1$ and $\alpha_{j} \in \Sigma\left(P_{0}\right) \cap \Sigma(\bar{Q})$. The latter set is contained in $\Sigma(\bar{Q}, \sigma)$, because $\Sigma(Q, \sigma \theta) \subseteq \Sigma\left(P_{0}\right)$. Let $X \in \mathfrak{a}^{+}(\bar{Q})$. Then it follows that the roots of $\Sigma(\bar{Q}, \sigma)$ are positive on the element $Y=X+\sigma(X)$ of $\mathfrak{a}_{\mathfrak{h}}$. Hence $\mu(Y)>0$; in particular $\mu \neq 0$. We thus see that

$$
\left[\mathfrak{n}_{P_{0}} U\left(\mathfrak{n}_{P_{0}}\right)\right]^{\mathfrak{a}_{\mathfrak{h}}}=\left[\left(\mathfrak{n}_{P_{0}} \cap \mathfrak{n}_{Q}\right) U\left(\mathfrak{n}_{P_{0}}\right)\right]^{\mathfrak{a}_{\mathfrak{h}}} .
$$

The result follows.

Remark 6.8. In view of the PBW theorem, the map $\mu$ is entirely determined either by the description in (a), or by the description in (b). For h-extreme $Q$ the proof of (b) is basically a reformulation of the argument given in the proof of AFJS12, Lemma 2.4].

Let $w \in \mathscr{W}$ (see the definition preceding Lemma 5.2). Then $\operatorname{Ad}(w)$ preserves $\mathfrak{m}$ and $\mathfrak{a}_{\mathfrak{q}}$. The action of $\operatorname{Ad}(w)$ on $\mathfrak{m}$ and $\mathfrak{a}_{\mathfrak{q}}$ induces an isomorphism of algebras

$$
\operatorname{Ad}(w): \mathbb{D}\left(M / H_{M}\right) \otimes \mathbb{D}\left(A_{\mathfrak{q}}\right) \rightarrow \mathbb{D}\left(M / w H_{M} w^{-1}\right) \otimes \mathbb{D}\left(A_{\mathfrak{q}}\right) .
$$

Accordingly, we define the algebra embedding

$$
\mu_{w}: \mathbb{D}(G / H) \rightarrow \mathbb{D}\left(M / w H_{M} w^{-1}\right) \otimes \mathbb{D}\left(A_{\mathfrak{q}}\right)
$$

by

$$
\mu_{w}:=\operatorname{Ad}(w) \circ \mu .
$$

Let $\left(\tau, V_{\tau}\right)$ be a finite dimensional unitary representation of $K$. For each $w \in$ $\mathscr{W}$ the natural action of $\mathbb{D}\left(M / w H_{M} w^{-1}\right)$ on $C^{\infty}\left(M / w H_{M} w^{-1}: \tau_{M}^{0}\right)$ induces an algebra homomorphism

$$
r_{w}: \mathbb{D}\left(M / w H_{M} w^{-1}\right) \rightarrow \operatorname{End}\left(C^{\infty}\left(M / w H_{M} w^{-1}: \tau_{M}^{0}\right)\right)
$$


In the following we will view $\operatorname{End}\left(\mathscr{A}_{M, 2}(\tau)\right) \otimes \mathbb{D}\left(A_{\mathfrak{q}}\right)$ as the algebra of invariant differential operators with coefficients in $\operatorname{End}\left(\mathscr{A}_{M, 2}(\tau)\right)$, which naturally acts on $C^{\infty}\left(A_{\mathfrak{q}}\right) \otimes \mathscr{A}_{M, 2}(\tau)$. Accordingly, we define the algebra homomorphism

$$
\mu(\cdot: \tau): \mathbb{D}(G / H) \rightarrow \operatorname{End}\left(\mathscr{A}_{M, 2}(\tau)\right) \otimes \mathbb{D}\left(A_{\mathfrak{q}}\right)
$$

by

$$
(\mu(D: \tau) \Psi)_{w}=\left[\left(r_{w} \otimes I\right) \circ \mu_{w}(D)\right] \Psi_{w} \quad\left(\Psi \in C^{\infty}\left(A_{\mathfrak{q}}\right) \otimes \mathscr{A}_{M, 2}(\tau), w \in \mathscr{W}\right)
$$

for $D \in \mathbb{D}(G / H)$.

Proposition 6.9. Let $Q \in \mathscr{P}(A)$. If $D \in \mathbb{D}(G / H)$ and $\phi \in C_{c}^{\infty}(G / H: \tau)$, then

$$
\mathcal{H}_{Q, \tau}(\phi)=\mu(D: \tau) \mathcal{H}_{Q, \tau} \phi .
$$

Proof. Let $v \in \mathscr{W}$. Fix $P_{0} \in \mathscr{P}_{\sigma}\left(A_{\mathfrak{q}}\right)$ such that $\Sigma(Q, \sigma \theta) \subseteq \Sigma\left(P_{0}\right)$. In view of Lemma 1.4 there exists a unique $P \in \mathscr{P}_{\sigma}(A)$ such that $Q \preceq P \subseteq P_{0}$. Then $\Sigma\left(Q^{v}, \sigma \theta\right) \subseteq$ $\Sigma\left(P^{v}, \sigma \theta\right)=\Sigma\left(P_{0}^{v}\right)$. Let $D \in \mathbb{D}(G / H)$ and let $u$ and $u_{0}$ be associated with $D$ as in Lemma 6.7, but with $P_{0}^{v}, Q^{v}$ in place of $P_{0}, Q$. Then

$$
u-u_{0} \in\left(\mathfrak{n}_{P_{0}^{v}} \cap \mathfrak{n}_{Q^{v}}\right) U(\mathfrak{g})+U(\mathfrak{g}) \mathfrak{h}
$$

and

$$
\mu(D)=d_{v}^{-1} \circ D_{0} \circ d_{v},
$$

where $D_{0}=R_{u_{0}}$ and $d_{v}(a)=a^{v^{-1} \rho_{P_{0}}}$, for $a \in A_{\mathfrak{q}}$; see Remark 6.6.

Let $X \in \mathfrak{a}_{\mathfrak{q}}$ be such that $\alpha(X)>0$ for every $\alpha \in \Sigma\left(P_{0}^{v}\right)$. Then $X$ satisfies (2.1) for the pair $\left(P^{v}, Q^{v}\right)$. By Lemma 2.3 we infer that $N_{Q^{v}, X}=N_{Q^{v}} \cap N_{P^{v}}=N_{Q^{v}} \cap N_{P_{0}^{v}}$. By Definition 6.1 and Corollary 2.2 it now follows that

$$
\left(\mathcal{H}_{Q, \tau} \phi(a)\right)_{v}(m)=a^{\rho_{Q}-\rho_{Q, \mathfrak{h}}} \int_{N_{Q^{v} \cap N_{P_{0}^{v}}}} \phi(\text { mavn }) d n
$$

for all $\phi \in C_{c}^{\infty}(G / H: \tau), m \in M$ and $a \in A_{\mathfrak{q}}$. In the integral on the right-hand side, the function $\phi$ should be viewed as a function in $C^{\infty}(G) \otimes V_{\tau}$ of compact support modulo $H$, i.e., with support in $G$ that has compact image in $G / H$. Accordingly, we define

$$
T \phi(m, a):=a^{\rho_{Q}-\rho_{Q, \mathfrak{h}}} \int_{N_{Q^{v} \cap N_{P_{0}^{v}}}} \phi(\text { mavn }) d n \quad\left((m, a) \in M \times A_{\mathfrak{q}}\right),
$$

for any such function $\phi$. Note that $T \phi \in C^{\infty}\left(M \times A_{\mathfrak{q}}\right) \otimes V_{\tau}$. It is readily verified that

$$
T\left(R_{Z} \phi\right)(m, a)=0
$$

for $\phi \in C_{c}^{\infty}(G / H: \tau)$ and $Z \in\left(\mathfrak{n}_{P_{0}^{v}} \cap \mathfrak{n}_{Q^{v}}\right) U(\mathfrak{g})+U(\mathfrak{g}) \mathfrak{h}$. Therefore,

$$
\left(\mathcal{H}_{Q, \tau} D \phi(a)\right)_{v}(m)=T\left(R_{u_{0}} \phi\right)(m, a) .
$$

For any function $\phi \in C^{\infty}(G: \tau)$ of compact support modulo $H$ we have

$$
\begin{aligned}
T(\phi)(m, a) & =a^{\rho_{Q}-\rho_{Q, \mathfrak{h}}} \int_{N_{Q} \cap N_{P_{0}}} \phi(\operatorname{manv}) d n \\
& =\left.a^{\rho_{Q}-\rho_{Q, \mathfrak{h}}}|\operatorname{det} \operatorname{Ad}(a)|_{\mathfrak{n}_{Q} \cap \mathfrak{n}_{P_{0}}}\right|^{-1} \int_{N_{Q} \cap N_{P_{0}}} \phi(n m a v) d n
\end{aligned}
$$


for $(m, a) \in M \times A_{\mathfrak{q}}$. Since

$$
\begin{aligned}
& \left.a^{\rho_{Q}-\rho_{Q, \mathfrak{h}}}|\operatorname{det} \operatorname{Ad}(a)|_{\mathfrak{n}_{Q} \cap \mathfrak{n}_{P_{0}}}\right|^{-1}=\left.\left.|\operatorname{det} \operatorname{Ad}(a)|_{\mathfrak{n}_{Q} \cap \mathfrak{n}_{P_{0}}}\right|^{-\frac{1}{2}}|\operatorname{det} \operatorname{Ad}(a)| \mathfrak{n}_{Q} \cap \theta \mathfrak{n}_{P_{0}}\right|^{\frac{1}{2}} \\
& =\left.\left.|\operatorname{det} \operatorname{Ad}(a)|_{\mathfrak{n}_{Q} \cap \mathfrak{n}_{P_{0}}}\right|^{-\frac{1}{2}}|\operatorname{det} \operatorname{Ad}(a)|_{\theta \mathfrak{n}_{Q} \cap \mathfrak{n}_{P_{0}}}\right|^{-\frac{1}{2}} \\
& =a^{-\rho_{P_{0}}} \text {, }
\end{aligned}
$$

we infer, writing $d(a)=a^{\rho_{P_{0}}}$, that

$$
T \phi(m, a)=d(a)^{-1} \int_{N_{Q} \cap N_{P_{0}}} \phi\left(n v v^{-1} m a v\right) d n .
$$

Now let $\phi \in C_{c}^{\infty}(G / H)$, so that $T \phi \in C^{\infty}\left(M / v H_{M} v^{-1} \times A_{\mathfrak{q}}\right) \otimes V_{\tau}$. Then

$$
\begin{aligned}
T\left(R_{u_{0}} \phi\right)(m, a) & =\left[d^{-1} \circ R_{\operatorname{Ad}(v) u_{0}} \circ d\right](T \phi)(m, a) \\
& =\left[d^{-1} \circ \operatorname{Ad}(v)\left(R_{u_{0}}\right) \circ d\right](T \phi)(m, a) \\
& =\operatorname{Ad}(v)\left[d_{v}^{-1} \circ R_{u_{0}} \circ d_{v}\right](T \phi)(m, a) \\
& =\mu_{v}(D)(T \phi)(m a) .
\end{aligned}
$$

In view of (6.16), we finally conclude that

$$
\begin{aligned}
\left(\mathcal{H}_{Q, \tau} \phi(a)\right)_{v}(m) & =\mu_{v}(D)(T \phi)(m a) \\
& =\left(\left[\left(r_{w} \otimes I\right) \circ \mu_{v}(D)\right]\left(\mathcal{H}_{Q, \tau} \phi\right)_{v}(a)\right)(m) \\
& =\left[\mu(D: \tau)\left(\mathcal{H}_{Q, \tau} \phi\right)(a)\right]_{v}(m) .
\end{aligned}
$$

\section{Extension to the Schwartz SPACE}

Throughout this section, we assume that $Q \in \mathscr{P}(A)$ and that $P_{0}$ is a minimal $\sigma \theta$-stable parabolic subgroup that contains $A$ and satisfies $\Sigma(Q, \sigma \theta) \subseteq \Sigma\left(P_{0}\right)$; see Lemma 1.4.

We define

$$
\mathfrak{a}_{\mathfrak{q}}^{*+}\left(P_{0}\right):=\left\{\lambda \in \mathfrak{a}_{\mathfrak{q}}^{*}:\langle\lambda, \alpha\rangle>0 \forall \alpha \in \Sigma\left(P_{0}\right)\right\}
$$

and

$$
A_{\mathfrak{q}}^{+}\left(P_{0}\right)=\left\{a \in A_{\mathfrak{q}}: a^{\alpha}>1 \forall \alpha \in \Sigma\left(P_{0}\right)\right\}
$$

7.1. Tempered term of the $\tau$-spherical Harish-Chandra transform. Let $\left(\tau, V_{\tau}\right)$ be a finite dimensional unitary representation of $K$ as before. It is convenient to denote by $E(Q: \cdot)$ the meromorphic map $\mathfrak{a}_{\mathfrak{q} C}^{*} \rightarrow \operatorname{Hom}\left(\mathscr{A}_{M, 2}, C^{\infty}(G / H: \tau)\right)$ given by

$$
E(Q: \lambda) \psi=E(Q: \psi: \lambda) \quad\left(\lambda \in \mathfrak{a}_{\mathfrak{q} \mathbb{C}}^{*}, \psi \in \mathscr{A}_{M, 2}(\tau)\right) .
$$

By Proposition [5.8, the singular locus of $E(Q:-\cdot)$ equals the union of a locally finite collection $\operatorname{Hyp}(Q, \tau)$ of hyperplanes of the form $\left\{\lambda \in \mathfrak{a}_{\mathfrak{q} \mathbb{C}}^{*}:\langle\lambda, \alpha\rangle=c\right\}$ with $\alpha \in \Sigma \backslash \mathfrak{a}_{\mathfrak{h}}^{*}$ and $c \in \mathbb{R}$. Each such hyperplane $H$ can be written as $H:=\mu+\alpha_{\mathbb{C}}^{\perp}$, where $\mu \in \mathfrak{a}_{\mathfrak{q}}^{*}$ is real, and where $\alpha_{\mathbb{C}}^{\perp}$ denotes the complexification of the real hyperplane $\alpha^{\perp} \subset \mathfrak{a}_{\mathfrak{q}}^{*}$. We note that $H_{\mathbb{R}}:=H \cap \mathfrak{a}_{\mathfrak{q}}^{*}$ equals $\mu+\alpha^{\perp}$ and that $H$ may be viewed as the complexification of $H_{\mathbb{R}}$. Moreover, we agree to write

$$
\operatorname{Hyp}_{\mathbb{R}}(Q, \tau):=\left\{H_{\mathbb{R}}: H \in \operatorname{Hyp}(Q, \tau)\right\} .
$$


We note that for $\mu \in \mathfrak{a}_{\mathfrak{q}}^{*+}\left(P_{0}\right) \backslash \bigcup \operatorname{Hyp}_{\mathbb{R}}(Q, \tau)$ the function $E(Q:-\cdot)$ is regular on $\mu+i \mathfrak{a}_{\mathfrak{q}}^{*}$. Furthermore, if $\phi \in C_{c}^{\infty}(G / H: \tau)$ then from the Paley-Wiener type estimate (5.30) in Proposition 5.8 we infer that

$$
\lambda \mapsto \mathfrak{F}_{Q, \tau} \phi(\lambda) a^{\lambda}
$$

is integrable on $\mu+i \mathfrak{a}_{\mathfrak{q}}^{*}$ for every $a \in A_{\mathfrak{q}}$. In view of the estimates in the same proposition, it follows by application of Cauchy's integral formula that the map

$$
\mathfrak{a}_{\mathfrak{q}}^{*+}\left(P_{0}\right) \backslash \bigcup \operatorname{Hyp}_{\mathbb{R}}(Q, \tau) \ni \mu \mapsto \int_{\mu+i \mathfrak{a}_{\mathfrak{q}}^{*}} \mathfrak{F}_{Q, \tau} \phi(\lambda) a^{\lambda} d \lambda
$$

is locally constant. Therefore, it is constant on each connected component of $\mathfrak{a}_{\mathfrak{q}}^{*+}\left(P_{0}\right) \backslash \bigcup \operatorname{Hyp}_{\mathbb{R}}(Q, \tau)$. Here $d \lambda$ denotes the choice of (real) measure on $\mu+i \mathfrak{a}_{\mathfrak{q}}^{*}$ obtained by transferring $(2 \pi)^{-\operatorname{dim} \mathfrak{a}_{\mathfrak{q}}}$ times the Lebesgue measure on $\mathfrak{a}_{\mathfrak{q}}^{*}$ under the $\operatorname{map} \lambda \mapsto \mu+i \lambda$.

Since $E(Q:-\cdot)$ is holomorphic on an open neighborhood of the closed convex set $-\Upsilon_{Q}$ (see (5.3) $)$, it follows that there exists a connected component $C_{1}$ of $\mathfrak{a}_{\mathfrak{q}}^{*+}\left(P_{0}\right) \backslash \bigcup \operatorname{Hyp}_{\mathbb{R}}(Q, \tau)$ such that

$$
C_{1} \supseteq \mathfrak{a}_{\mathfrak{q}}^{*+}\left(P_{0}\right) \cap\left(-\Upsilon_{Q}\right) .
$$

Lemma 7.1. Let $\mu \in C_{1}$. Then

$$
\mathcal{H}_{Q, \tau}(\phi)(a)=\int_{\mu+i \mathfrak{a}_{\mathfrak{q}}^{*}} \mathfrak{F}_{Q, \tau} \phi(\lambda) a^{\lambda} d \lambda \quad\left(a \in A_{\mathfrak{q}}\right) .
$$

Proof. As the expression on the right-hand side of the equation is independent of $\mu \in C_{1}$, we may assume that $\mu \in-\Upsilon_{Q}$. Then in view of Proposition 6.4,

$$
\int_{\mu+i \mathfrak{a}_{\mathfrak{q}}^{*}} \mathfrak{F}_{Q, \tau} \phi(\lambda) a^{\lambda} d \lambda=\int_{i \mathfrak{a}_{\mathfrak{q}}^{*}} \mathcal{F}_{A_{\mathfrak{q}}}\left(\mathcal{H}_{Q, \tau}(\phi)\right)(\mu+\lambda) a^{\mu} a^{\lambda} d \lambda=\mathcal{H}_{Q, \tau}(a),
$$

where the latter equality is valid by application of the Fourier inversion formula.

We intend to analyze $\mathcal{H}_{Q, \tau}(\phi)$ by applying a contour shift to the integral on the right-hand side of (7.1) with $\mu$ tending to zero in a suitable way. This will result in residual terms. In the $\sigma$-split rank one case, these are point residues, which will be analyzed in the next section. For general $\sigma$-split rank, one may hope to analyze them by using a multi-dimensional residue calculus in the spirit of vdBS00].

Clearly, there exists a unique connected component $C_{0}$ of $\mathfrak{a}_{\mathfrak{q}}^{*+}\left(P_{0}\right) \backslash \cup \operatorname{Hyp}_{\mathbb{R}}(Q, \tau)$ with $0 \in \bar{C}_{0}$. For $\phi \in C_{c}^{\infty}(G / H: \tau)$ we define $\mathcal{I}_{Q, \tau} \phi: A_{\mathfrak{q}} \rightarrow \mathscr{A}_{M, 2}(\tau)$ by

$$
\mathcal{I}_{Q, \tau} \phi(a)=\lim _{\epsilon \downarrow 0} \int_{\epsilon \nu+i \mathfrak{a}_{\mathfrak{q}}^{*}} \mathfrak{F}_{Q, \tau} \phi(\lambda) a^{\lambda} d \lambda \quad\left(a \in A_{\mathfrak{q}}\right) .
$$

Here $\nu$ is any choice of element of $\mathfrak{a}_{\mathfrak{q}}^{*+}\left(P_{0}\right)$; the definition is independent of this choice and

$$
\mathcal{I}_{Q, \tau} \phi(a)=\int_{\mu+i \mathfrak{a}_{\mathfrak{q}}^{*}} \mathfrak{F}_{Q, \tau} \phi(\lambda) a^{\lambda} d \lambda
$$

for $\mu \in C_{0}$. The function $\mathcal{I}_{Q, \tau} \phi: A_{\mathfrak{q}} \rightarrow \mathscr{A}_{M, 2}(\tau)$ will be called the tempered term of the Harish-Chandra transform.

We define

$$
C_{\text {temp }}^{\infty}\left(A_{\mathfrak{q}}\right)
$$


to be the space of smooth functions on $A_{\mathfrak{q}}$ which are tempered as distributions on $A_{\mathfrak{q}}$, viewed as a Euclidean space, i.e., belong to the dual of the Euclidean Schwartz space $\mathscr{S}\left(A_{\mathfrak{q}}\right)$. We equip the space (7.3) with the coarsest locally convex topology such that the inclusion maps into $C^{\infty}\left(A_{\mathfrak{q}}\right)$ and $\mathscr{S}^{\prime}\left(A_{\mathfrak{q}}\right)$ are both continuous. Here $C^{\infty}\left(A_{\mathfrak{q}}\right)$ and $\mathscr{S}\left(A_{\mathfrak{q}}\right)$ are equipped with the usual Fréchet topologies and $\mathscr{S}^{\prime}\left(A_{\mathfrak{q}}\right)$ is equipped with the strong dual topology.

Proposition 7.2. If $\phi \in C_{c}^{\infty}(G / H: \tau)$, then $\mathcal{I}_{Q, \tau} \phi \in C_{\text {temp }}^{\infty}\left(A_{\mathfrak{q}}\right) \otimes \mathscr{A}_{M, 2}(\tau)$. The map $C_{c}^{\infty}(G / H: \tau) \rightarrow C_{\text {temp }}^{\infty}\left(A_{\mathfrak{q}}\right) \otimes \mathscr{A}_{M, 2}(\tau)$ thus obtained has a unique extension to a continuous linear map

$$
\mathcal{I}_{Q, \tau}: \mathscr{C}(G / H: \tau) \rightarrow C_{\mathrm{temp}}^{\infty}\left(A_{\mathfrak{q}}\right) \otimes \mathscr{A}_{M, 2}(\tau) .
$$

Proof. Let $B \subseteq \mathfrak{a}_{\mathfrak{q}}^{*}$ be a bounded neighborhood of 0 . Let $p \in \Pi_{\Sigma, \mathbb{R}}\left(\mathfrak{a}_{\mathfrak{q}}^{*}\right)$ be as in Proposition $5.5(\mathrm{c})$. Then $p(-\cdot)$ belongs to $\Pi_{\Sigma, \mathbb{R}}\left(\mathfrak{a}_{\mathfrak{q}}^{*}\right)$, hence admits a decomposition as a product of a polynomial from $\Pi_{\Sigma, \mathbb{R}}\left(\mathfrak{a}_{\mathfrak{q}}^{*}\right)$ which vanishes nowhere on $i \mathfrak{a}_{\mathfrak{q}}^{*}$ and a polynomial $p_{h} \in \Pi_{\Sigma, \mathbb{R}}\left(\mathfrak{a}_{\mathfrak{q}}^{*}\right)$ which is homogeneous. Then $\lambda \mapsto p_{h}(-\lambda) C_{\bar{P}_{0} \mid Q}(1: \lambda)$ is holomorphic on an open neighborhood of $i \mathfrak{a}_{\mathfrak{q}}^{*}$ in $\mathfrak{a}_{\mathfrak{q} \mathfrak{C}}^{*}$.

According to vdBS97c, Lemma 6.2] the Fourier transform $\mathcal{F}_{\bar{P}_{0}}$ extends to a continuous linear map from $\mathscr{C}(G / H: \tau)$ to $\mathscr{S}\left(i \mathfrak{a}_{\mathfrak{q}}^{*}\right) \otimes \mathscr{A}_{M, 2}(\tau)$. Hence, in view of Proposition [5.7. also the map $\phi \mapsto p_{h} \mathfrak{F}_{Q, \tau} \phi$ extends to a continuous linear map $\mathscr{C}(G / H: \tau) \rightarrow \mathscr{S}\left(i \mathfrak{a}_{\mathfrak{q}}^{*}\right) \otimes \mathscr{A}_{M, 2}(\tau)$ and for all $\phi \in \mathscr{C}(G / H: \tau)$ we have

$$
\left[p_{h} \mathfrak{F}_{Q, \tau} \phi\right](\lambda)=p_{h}(\lambda) C_{\bar{P}_{0}: Q}(1:-\bar{\lambda})^{*} \mathcal{F}_{\bar{P}_{0}}(\phi)(\lambda) \quad\left(\lambda \in i \mathfrak{a}_{\mathfrak{q}}^{*}\right) .
$$

We now see that, for $\phi \in \mathscr{C}(G / H: \tau)$,

$$
\mathcal{K}_{Q, \tau} \phi(a):=\int_{i \mathfrak{a}_{\mathfrak{q}}^{*}} p_{h}(\lambda) \mathfrak{F}_{Q, \tau} \phi(\lambda) a^{\lambda} d \lambda \quad\left(a \in A_{\mathfrak{q}}\right)
$$

defines an element of $\mathscr{S}\left(A_{\mathfrak{q}}\right) \otimes \mathscr{A}_{M, 2}(\tau)$ and the map

$$
\mathcal{K}_{Q, \tau}: \mathscr{C}(G / H: \tau) \rightarrow \mathscr{S}\left(A_{\mathfrak{q}}\right) \otimes \mathscr{A}_{M, 2}(\tau)
$$

thus obtained is continuous linear.

Let $\nu \in \mathfrak{a}_{\mathfrak{q}}^{*+}\left(P_{0}\right)$. It follows from [Hör03, Thm. 3.1.15] that the limit

$$
v(f):=\lim _{\epsilon \downarrow 0} \int_{i \mathfrak{a}_{\mathfrak{q}}^{*}} \frac{f(\lambda)}{p_{h}(\lambda+\epsilon \nu)} d \lambda
$$

exists for every $f \in \mathscr{S}\left(i \mathfrak{a}_{\mathfrak{q}}^{*}\right)$, and that accordingly $v$ defines a distribution on $i \mathfrak{a}_{\mathfrak{q}}^{*}$. This distribution is homogeneous, hence tempered; see [Hör03, Thm. 7.1.18]. Put $u:=\mathcal{F}_{A_{\mathfrak{q}}}^{-1} v$. Then $u$ is a tempered distribution on $A_{\mathfrak{q}}$, hence the convolution operator $f \mapsto u * f$ defines a continuous linear map $\mathscr{S}\left(A_{\mathfrak{q}}\right) \rightarrow C_{\text {temp }}^{\infty}\left(A_{\mathfrak{q}}\right)$. Thus, to finish the proof, it suffices to prove the claim that for every $\phi \in C_{c}^{\infty}(G / H: \tau)$,

$$
\mathcal{I}_{Q, \tau} \phi=u * \mathcal{K}_{Q, \tau} \phi .
$$

We set $\Phi:=p_{h} \mathfrak{F}_{Q, \tau} \phi$ and note that $\mathcal{K}_{Q, \tau} \phi=\mathcal{F}_{A_{\mathfrak{q}}}^{-1} \Phi$. Therefore,

$$
u * \mathcal{K}_{Q, \tau} \phi=\left(\mathcal{F}_{A_{\mathfrak{q}}}^{-1} v\right) * \mathcal{F}_{A_{\mathfrak{q}}}^{-1} \Phi=\mathcal{F}_{A_{\mathfrak{q}}}^{-1}(\Phi v) .
$$


Let $\psi \in C_{c}^{\infty}\left(i \mathfrak{a}_{\mathfrak{q}}^{*}\right)$. Then

$$
\begin{aligned}
\Phi v(\psi) & =\lim _{\epsilon \downarrow 0} \int_{i \mathfrak{a}_{\mathfrak{q}}^{*}} \frac{\Phi(\lambda) \psi(\lambda)}{p_{h}(\lambda+\epsilon \nu)} d \lambda \\
& =\lim _{\epsilon \downarrow 0}\left(\int_{i \mathfrak{a}_{\mathfrak{q}}^{*}} \frac{\Phi(\lambda+\epsilon \nu) \psi(\lambda)}{p_{h}(\lambda+\epsilon \nu)} d \lambda-\int_{i \mathfrak{a}_{\mathfrak{q}}^{*}}\left(\int_{0}^{\epsilon} \partial_{t} \Phi(\lambda+t \nu) d t\right) \frac{\psi(\lambda)}{p_{h}(\lambda+\epsilon \nu)} d \lambda\right) \\
& =\lim _{\epsilon \downarrow 0}\left(\int_{i \mathfrak{a}_{\mathfrak{q}}^{*}} \mathfrak{F}_{Q, \tau} \phi(\lambda+\epsilon \nu) \psi(\lambda) d \lambda-\int_{0}^{\epsilon} \int_{i \mathfrak{a}_{\mathfrak{q}}^{*}} \frac{\partial_{t} \Phi(\lambda+t \nu) \psi(\lambda)}{p_{h}(\lambda+\epsilon \nu)} d \lambda d t\right) .
\end{aligned}
$$

The function

$$
F:(t, \epsilon) \mapsto \int_{i \mathfrak{a}_{\mathfrak{q}}^{*}} \frac{\partial_{t} \Phi(\lambda+t \nu) \psi(\lambda)}{p_{h}(\lambda+\epsilon \nu)} d \lambda
$$

is continuous on $[0,1] \times] 0,1]$. Moreover, since $f: t \mapsto \partial_{t} \Phi(\cdot+t \nu) \psi(\cdot)$ is a continuous function $[0,1] \rightarrow C_{c}^{\infty}\left(i \mathfrak{a}_{\mathfrak{q}}^{*}\right)$, it follows that in the Banach space $C([0,1])$,

$$
F(\cdot, \epsilon) \rightarrow v(f(\cdot)) \quad(\epsilon \downarrow 0) .
$$

We thus see that $F$ extends continuously to $[0,1] \times[0,1]$. This in turn implies that

$$
\lim _{\epsilon \downarrow 0} \int_{0}^{\epsilon} F(t, \epsilon) d t=0
$$

hence

$$
\Phi v(\psi)=\lim _{\epsilon \downarrow 0} \int_{i \mathfrak{a}_{\mathfrak{q}}^{*}} \mathfrak{F}_{Q, \tau} \phi(\lambda+\epsilon \nu) \psi(\lambda) d \lambda .
$$

Now let $\chi \in C_{c}^{\infty}\left(A_{\mathfrak{q}}\right)$. Then

$$
\begin{aligned}
\left(u * \mathcal{K}_{Q, \tau} \phi\right)(\chi) & =\Phi v\left(\lambda \mapsto \int_{A_{\mathfrak{q}}} \chi(a) a^{\lambda} d a\right) \\
& =\lim _{\epsilon \downarrow 0} \int_{i \mathfrak{a}_{\mathfrak{q}}^{*}} \int_{A_{\mathfrak{q}}} \mathfrak{F}_{Q, \tau} \phi(\lambda+\epsilon \nu) \chi(a) a^{\lambda} d a d \lambda \\
& =\lim _{\epsilon \downarrow 0} \int_{A_{\mathfrak{q}}}\left(\int_{i \mathfrak{a}_{\mathfrak{q}}^{*}} \mathfrak{F}_{Q, \tau} \phi(\lambda+\epsilon \nu) a^{\lambda+\epsilon \nu} d \lambda\right) a^{-\epsilon \nu} \chi(a) d a .
\end{aligned}
$$

If $\epsilon$ is sufficiently small, then

$$
\mathcal{I}_{Q, \tau} \phi(a)=\int_{i \mathfrak{a}_{\mathfrak{q}}^{*}} \mathfrak{F}_{Q, \tau} \phi(\lambda+\epsilon \nu) a^{\lambda+\epsilon \nu} d \lambda
$$

(see (7.2) $)$, hence

$$
\left(u * \mathcal{K}_{Q, \tau} \phi\right)(\chi)=\lim _{\epsilon \downarrow 0} \int_{A_{\mathfrak{q}}} \mathcal{I}_{Q, \tau} \phi(a) a^{-\epsilon \nu} \chi(a) d a=\int_{A_{\mathfrak{q}}} \mathcal{I}_{Q, \tau} \phi(a) \chi(a) d a .
$$

This establishes the claim (7.7).

Remark 7.3. Assume that the Eisenstein integral $E(Q:-\cdot)=E_{\tau}(Q:-\cdot)$ is holomorphic on $] 0,1] \cdot \xi$ for an element $\xi \in C_{1}$. Then the chambers $C_{0}$ and $C_{1}$ are equal, and it follows that $\mathcal{H}_{Q, \tau} \phi=\mathcal{I}_{Q, \tau} \phi$. In view of Proposition 7.2 . the spherical Harish-Chandra transform $\mathcal{H}_{Q, \tau}$ extends to a continuous linear map $\mathscr{C}(G / H: \tau) \rightarrow C_{\text {temp }}^{\infty}\left(A_{\mathfrak{q}}\right) \otimes \mathscr{A}_{M, 2}(\tau)$.

Now assume that the above condition of holomorphy is fulfilled for $\left(\tau, V_{\tau}\right)$ equal to the trivial representation $(1, \mathbb{C})$ of $K$. Then $\mathscr{C}(G / H: \tau)=\mathscr{C}(G / H)^{K}$ and 
$\mathscr{A}_{M, 2}(\tau)=\mathbb{C}^{\mathscr{W}}$ and it follows by application of Lemma 6.2 that the restriction of $\mathcal{H}_{Q}$ to $C_{c}^{\infty}(G / H)^{K}$ extends to a continuous linear map $\mathscr{C}(G / H)^{K} \rightarrow C^{\infty}\left(L / H_{L}\right)^{M}$. By application of Proposition 4.6 it now follows that $\mathcal{H}_{Q}$ extends to a continuous linear map $\mathscr{C}(G / H) \rightarrow C^{\infty}\left(L / H_{L}\right)$ and is given by absolutely convergent integrals. Moreover, the image of $\mathcal{H}_{Q}$ consists of tempered functions.

Finally, assume that $\Sigma_{-}(Q)=\emptyset$. Then $\Upsilon_{Q}=\mathfrak{a}_{\mathfrak{q C}}^{*}$. This implies that $\mathfrak{F}_{Q, \tau} \phi$ is holomorphic on $\mathfrak{a}_{\mathfrak{q} \mathbb{C}}^{*}$ for every $\phi \in C_{c}^{\infty}(G / H: \tau)$. Now a stronger statement can be obtained than in the more general setting above. The polynomial $p$ in the proof for Proposition 7.2 can be taken equal to the constant function 1 . The distribution $u$ is then equal to the Dirac measure at the origin of $i \mathfrak{a}_{\mathfrak{q}}^{*}$ and as a consequence, $\mathcal{I}_{Q, \tau}$ is equal to $\mathcal{K}_{Q, \tau}$. In particular, it follows that $\mathcal{H}_{Q, 1}$ extends to a continuous linear map $\mathscr{C}(G / H)^{K} \rightarrow \mathscr{C}\left(L / H_{L}\right)^{M}$ and is given by absolutely convergent integrals. In view of Lemma 6.2 it follows that $\mathcal{H}_{Q}$ maps $\mathscr{C}(G / H)^{K}$ continuous linearly into $\mathscr{C}\left(L / H_{L}\right)^{M}$.

We will now apply domination to show that in this case $\mathcal{H}_{Q}$ is a continuous linear map from $\mathscr{C}(G / H)$ to $\mathscr{C}\left(L / H_{L}\right)$. In the above we established already that for $\phi \in \mathscr{C}(G / H)$ the function $\mathcal{H}_{Q} \phi \in C^{\infty}\left(L / H_{L}\right)$ is given by absolutely convergent integrals. For the purpose of estimation, let $\varphi \mapsto \widehat{\varphi}$ be a map as in Proposition 4.2. Let $u \in U(\mathfrak{l})$. Then there exists a $u^{\prime} \in U(\mathfrak{l})$ such that $L_{u} \circ \delta_{Q}=\delta_{Q} \circ L_{u^{\prime}}$ on $C^{\infty}\left(L / H_{L}\right)$. Thus, for $\phi \in \mathscr{C}(G / H)$ we have

$$
L_{u} \mathcal{H}_{Q}(\phi)=\delta_{Q} L_{u^{\prime}} \mathcal{R}_{Q}(\phi)=\mathcal{H}_{Q}\left(L_{u^{\prime}} \phi\right),
$$

by equivariance of the Radon transform. Let $N \in \mathbb{N}$. There exists a continuous seminorm $\nu$ on $\mathscr{C}(G / H)$ such that for all $\phi \in \mathscr{C}(G / H)^{K}$ and $l \in L$,

$$
(1+\|l\|)^{N}\left|\mathcal{H}_{Q}(\phi)(l)\right| \leq \nu(\phi) .
$$

It now follows by application of Proposition 4.2 that there exists a continuous seminorm $\mu$ on $\mathscr{C}(G / H)$ such that

$$
\nu(\widehat{\phi}) \leq \mu(\phi) \quad(\phi \in \mathscr{C}(G / H)) .
$$

Combining the equality (7.8) with the estimates (7.9) and (7.10), we find

$$
\begin{aligned}
(1+\|l\|)^{N}\left|L_{u} \mathcal{H}_{Q}(\phi)(l)\right| & \leq(1+\|l\|)^{N} \mathcal{H}_{Q}\left(\left|L_{u^{\prime}} \phi\right|\right) \\
& \leq(1+\|l\|)^{N} \mathcal{H}_{Q}\left(\widehat{L_{u^{\prime}} \phi}\right) \\
& \leq \nu\left(\widehat{L_{u^{\prime}} \phi}\right) \leq \mu\left(L_{u^{\prime}} \phi\right) .
\end{aligned}
$$

This establishes the continuity.

Example 7.4 (Group case). We use the notation of Example 3.2. Assume that ' $P$ and ' $Q$ are minimal parabolic subgroups of ' $G$ containing ' $A$. Since $\Sigma_{-}\left({ }^{\prime} P \times{ }^{'} P\right)=$ $\emptyset$, the final analysis in Remark 7.3 applies to $\mathcal{H}^{\prime} P \times^{\prime} P$. Let ${ }^{\prime} \xi \in{ }^{\prime} \widehat{M}$ and define $\xi \in \widehat{M}$ by $\xi:={ }^{\prime} \xi \otimes{ }^{\prime} \xi^{\vee}$. For ${ }^{\prime} \lambda \in{ }^{\prime} \mathfrak{a}_{\mathbb{C}}^{*}$ we set $\lambda=\left({ }^{\prime} \lambda,-{ }^{\prime} \lambda\right) \in \mathfrak{a}_{\mathfrak{q} \mathbb{C}}^{*}$. Let $\left(\tau, V_{\tau}\right)$ be a finite dimensional unitary representation of $K={ }^{\prime} K \times{ }^{\prime} K$. We recall from vdBK14, Eqn. (8.16)] that the $C$-function $C\left({ }^{\prime} Q \times{ }^{\prime} Q:{ }^{\prime} P \times{ }^{\prime} Q: \lambda\right) \in \operatorname{End}\left(\mathscr{A}_{M, 2}(\tau)\right)$ is defined by the relation

$$
E\left({ }^{\prime} P \times{ }^{\prime} Q: \lambda\right)=E\left({ }^{\prime} Q \times{ }^{\prime} Q: \lambda\right) \circ C\left({ }^{\prime} Q \times{ }^{\prime} Q:{ }^{\prime} P \times{ }^{\prime} Q: \lambda\right) .
$$

It follows from vdBK14, Cor. 9.6, 9.8] that

$$
C\left({ }^{\prime} Q \times{ }^{\prime} Q:^{\prime} P \times{ }^{\prime} Q: \lambda\right) \psi_{f \otimes I_{\xi}}=\psi_{\left.\left[A\left({ }^{\prime} Q:{ }^{\prime} P: ' \xi:-{ }^{\prime} \lambda\right) \otimes I\right) f\right] \otimes I_{\xi}}
$$


for $\xi \in \widehat{M}$ and $f \in C^{\infty}(K: \xi: \tau)$. The intertwining operator $A\left({ }^{\prime} Q:{ }^{\prime} P:{ }^{\prime} \xi:^{'} \lambda\right)$ depends holomorphically on $\lambda=\left({ }^{\prime} \lambda,-{ }^{\prime} \bar{\lambda}\right)$ in the region

$$
U:=\left\{\lambda \in \mathfrak{a}_{\mathfrak{q} \mathbb{C}}^{*}:\left\langle{ }^{\prime} \lambda, \alpha\right\rangle>0 \text { for all } \alpha \in \Sigma\left({ }^{\prime} P\right) \cap \Sigma\left({ }^{\prime} \bar{Q}\right)\right\} .
$$

Clearly, $U$ is contained in $\mathfrak{a}_{\mathfrak{q} \mathbb{C}}^{*+}\left({ }^{\prime} P \times{ }^{\prime} \bar{P}\right)$. It follows that the $C$-function in (7.11) depends holomorphically on $\lambda \in-U$. From (7.11) it follows that

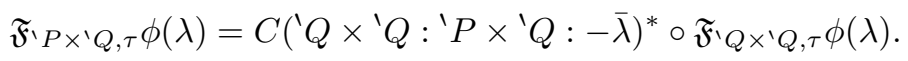

Hence, $\mathfrak{F}^{\prime} P \times^{\prime} Q \phi$ is holomorphic on $\mathfrak{a}_{\mathfrak{q}}^{*+}\left({ }^{\prime} P \times{ }^{\prime} \bar{P}\right)$ for every $\phi \in C_{c}^{\infty}(G / H: \tau)$. It

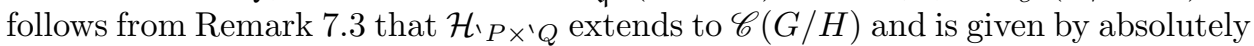
convergent integrals. Moreover, $\mathcal{H}_{\backslash P \times^{\prime} Q}$ maps $\mathscr{C}(G / H)$ to the space of smooth tempered functions on $L / H_{L}$.

The convergence of the integrals for $\mathcal{H}^{\prime} P \times^{\prime} Q$ also follows from combining Wal88, Thm. 7.2.1] and Wal92, Lemma 15.3.2].

Remark 7.5. We should inform the reader that Wal92, Lemma 15.3.2] has an additional assertion that a certain transform $f^{P}$ is of Schwartz behavior. However, the proof of this assertion is not correct. In fact, in the right-hand side of the inequality at the top of page 377 , a factor $\left(1+\left\|\log \left(a a_{1}\right)\right\|\right)^{2 d}$ is missing.

From the given proof it can be concluded that the map $f \mapsto f^{P}$ is given by absolutely convergent integrals, that it is continuous from $\mathscr{C}\left(N_{0} \backslash G: \chi\right)$ to $C^{\infty}\left(N_{0} \cap M_{P} \backslash M_{P}:\left.\chi\right|_{N_{0} \cap M_{P}}\right)$, and that its image consists of tempered smooth functions. However, the second part of Lemma 15.3.2 of Wal92 cannot be true in the generality stated. Indeed, combined with [Wal88, Thm. 7.2.1] the validity of the lemma would imply that $\mathcal{H}_{\backslash P \times^{\prime} \bar{P}}$ maps $\mathscr{C}(G / H)$ to $\mathscr{C}\left(L / H_{L}\right)$. The latter assertion is already incorrect for ${ }^{\prime} G=\mathrm{SL}(2, \mathbb{R})$. This is established in Lemma 7.6 below.

The parts of the proof of the Whittaker Plancherel formula in Wal92 that are affected by the mentioned error have been fixed by Wallach in Wal17.

Lemma 7.6. Let ${ }^{\prime} G=\mathbf{S L}(2, \mathbb{R})$ and let $\phi \in \mathscr{C}(G / H)$. Assume that $\phi \geq 0$ and

$$
\phi\left(\left(\begin{array}{cc}
0 & -1 \\
1 & 0
\end{array}\right), e\right)>0 \text {. }
$$

Then

$$
\liminf _{\substack{a \rightarrow \infty \\ a \in A_{\mathfrak{q}}^{+}\left({ }^{\top} P \times^{\prime} \bar{P}\right)}} \mathcal{H}_{\backslash P \times^{\prime} \bar{P}} \phi(a)>0 .
$$

Proof. For $t>0$ and $x, y \in \mathbb{R}$, we define

$$
a_{t}=\left(\begin{array}{cc}
e^{t} & 0 \\
0 & e^{-t}
\end{array}\right), \quad n_{x}=\left(\begin{array}{cc}
1 & x \\
0 & 1
\end{array}\right), \quad \bar{n}_{y}=\left(\begin{array}{cc}
1 & 0 \\
-y & 1
\end{array}\right) .
$$

To shorten notation, we write

$$
I(t):=\mathcal{H}_{{ }_{P \times} \times \bar{P}} \phi\left(a_{t / 2}, a_{-t / 2}\right) .
$$

Using the identification $G / H \stackrel{\widetilde{\sim}}{\rightarrow}$ ' $G$ induced by $(x, y) \mapsto x y^{-1}$ to view $\phi$ as a function on ' $G$ we obtain

$$
I(t)=e^{t} \int_{\mathbb{R}} \int_{\mathbb{R}} \phi\left(a_{t / 2} n_{x} \bar{n}_{y}^{-1} a_{t / 2}\right) d x d y=e^{t} \int_{\mathbb{R}} \int_{\mathbb{R}} \phi\left(\begin{array}{cc}
e^{t}(1+x y) & x \\
y & e^{-t}
\end{array}\right) d x d y .
$$

Let $0<\epsilon<1$ and $\eta>0$. We define the domain

$$
D_{t}:=\left\{(x, y) \in \mathbb{R}^{2}:-\epsilon<e^{t}(1+x y)<\epsilon, 1<y<1+\eta\right\} .
$$


Note that $(x, y) \in D_{t}$ if and only if

$$
1<y<1+\eta \quad \text { and } \quad \frac{-e^{-t} \epsilon-1}{y}<x<\frac{e^{-t} \epsilon-1}{y} .
$$

Hence, for $t>0$,

$$
D_{t} \subseteq R_{\epsilon, \eta}:=\left[-\epsilon-1, \frac{\epsilon-1}{1+\eta}\right] \times[1,1+\eta] .
$$

We note that $R_{\epsilon, \eta}$ tends to $\{(-1,1)\}$ for $\epsilon, \eta \downarrow 0$ and define

$$
C_{\epsilon, \eta}:=\left\{\left(\begin{array}{cc}
a & b \\
c & d
\end{array}\right) \in^{\prime} G:|a| \leq \epsilon,|d| \leq \epsilon,(b, c) \in R_{\epsilon, \eta}\right\} .
$$

Then $C_{\epsilon, \eta}$ is compact and tends to the singleton consisting of the matrix in (7.12). We may therefore take $\epsilon$ and $\eta$ so close to zero that the function $\phi$ is strictly positive on $C_{\epsilon, \eta}$. We thus obtain, for $t>-\log \epsilon$, that

$$
\begin{aligned}
I(t) & \geq e^{t} \int_{1}^{1+\eta} \int_{\frac{-e^{-t_{\epsilon-1}}}{y}}^{\frac{e^{-t_{\epsilon-1}}}{y}} \phi\left(\begin{array}{cc}
e^{t}(1+x y) & x \\
y & e^{-t}
\end{array}\right) d x d y \\
& \geq e^{t} \inf _{(u, v) \in D_{t}} \phi\left(\begin{array}{cc}
e^{t}(1+u v) & u \\
v & e^{-t}
\end{array}\right) \int_{1}^{1+\eta} \int_{\frac{-e^{-t_{\epsilon-1}}}{y}}^{\frac{e^{-t_{\epsilon-1}}}{y}} d x d y \\
& \geq 2 \epsilon \log (1+\eta) \inf _{C_{\epsilon, \eta}} \phi 0 .
\end{aligned}
$$

7.2. The residual term for spaces of split rank one. We retain the notation of the previous subsection. In particular, $Q \in \mathscr{P}(A)$ and $P_{0} \in \mathscr{P}_{\sigma}\left(A_{\mathfrak{q}}\right)$ is such that $\Sigma(Q, \sigma \theta) \subseteq \Sigma\left(P_{0}\right)$. As mentioned in the previous subsection, the difference between $\mathcal{H}_{Q, \tau} \phi$ and $\mathcal{I}_{Q, \tau} \phi$ is equal to a finite sum of residual integrals. These become point residues in case $\operatorname{dim} \mathfrak{a}_{\mathfrak{q}}=1$. For the rest of this subsection, we make an assumption.

Assumption. $G / H$ is of split rank one, i.e., $\operatorname{dim} \mathfrak{a}_{\mathfrak{q}}=1$.

By our assumption on the split rank, each hyperplane from the set $\operatorname{Hyp}(Q, \tau)$ defined in the beginning of Subsection 7.1 consists of a single point in $\mathfrak{a}_{\mathfrak{q}}^{*}$. Furthermore, the union $\bigcup \operatorname{Hyp}(Q, \tau)$ is a discrete subset of $\mathfrak{a}_{\mathfrak{q}}^{*}$, by Proposition 5.8 .

We define

$$
S_{Q, \tau}:=\mathfrak{a}_{\mathfrak{q}}^{*+}\left(P_{0}\right) \cap(\bigcup \operatorname{Hyp}(Q, \tau)) .
$$

Lemma 7.7. The set $S_{Q, \tau}$ is finite.

Proof. The Eisenstein integral $E(Q:-\cdot)$ is holomorphic on $-\widehat{\Upsilon}_{Q}$. The latter set contains a set of the form $\xi+\mathfrak{a}_{\mathfrak{q}}^{*+}\left(P_{0}\right)+i \mathfrak{a}_{\mathfrak{q}}^{*}$, with $\xi \in \mathfrak{a}_{\mathfrak{q}}^{*}$. Hence, $S_{Q, \tau}$ is contained in the set $\mathfrak{a}_{\mathfrak{q}}^{*+}\left(P_{0}\right) \backslash\left(\xi+\mathfrak{a}_{\mathfrak{q}}^{*+}\left(P_{0}\right)+i \mathfrak{a}_{\mathfrak{q}}^{*}\right)$ which is bounded. Since $S_{Q, \tau}$ is discrete, the result follows.

For a meromorphic function $f: \mathfrak{a}_{\mathfrak{q} \mathbb{C}}^{*} \rightarrow \mathbb{C}$ and a point $\mu \in \mathfrak{a}_{\mathfrak{q} \mathbb{C}}^{*}$ we define the residue

$$
\operatorname{Res}_{\lambda=\mu} \varphi(\lambda):=\operatorname{Res}_{z=0} \varphi(\mu+z \omega) .
$$

Here $\omega$ is the unique vector in $\mathfrak{a}_{\mathfrak{q}}^{*+}\left(P_{0}\right)$ of unit length (relative to the Killing form), $z$ is a variable in the complex plane, and the residue on the right-hand side of (7.13) is the usual residue from complex analysis, i.e., the coefficient of $z^{-1}$ in the Laurent expansion of $z \mapsto \varphi(\mu+z \omega)$ around $z=0$. 
Lemma 7.8. Let $\phi \in C_{c}(G / H: \tau)$. Then

$$
\mathcal{H}_{Q, \tau} \phi(a)=\mathcal{I}_{Q, \tau} \phi(a)+\sum_{\mu \in S_{Q, \tau}} \operatorname{Res}_{\lambda=\mu} a^{\lambda} \mathfrak{F}_{Q, \tau} \phi(\lambda) .
$$

Proof. By the chosen normalization of the measure $d \lambda$ on $\mu+i \mathfrak{a}_{\mathfrak{q}}^{*}$,

$$
\int_{\nu \omega+i \mathfrak{a}_{\mathfrak{q}}^{*}} \mathfrak{F}_{Q, \tau}(\phi)(\lambda) d \lambda=\frac{1}{2 \pi i} \int_{\nu+i \mathbb{R}} \mathfrak{F}_{Q, \tau}(\phi)(\tau \omega) d \tau .
$$

In view of the estimates (5.30) the result now follows by a straightforward application of the Cauchy integral formula.

Lemma 7.9. Let $\psi \in \mathscr{A}_{M, 2}(\tau)$ and $\mu \in S_{Q, \tau}$. Then for all $\phi \in C_{c}(G / H: \tau)$,

$$
\operatorname{Res}_{\lambda=\mu}\left\langle a^{\lambda} \mathfrak{F}_{Q, \tau}(\phi)(\lambda), \psi\right\rangle=a^{\mu}\left\langle\phi, \operatorname{Res}_{\tau}(Q: \mu: a: \cdot)(\psi)\right\rangle,
$$

where $\operatorname{Res}_{\tau}(Q: \mu)$ is the function $A_{\mathfrak{q}} \times G / H \rightarrow \operatorname{Hom}\left(\mathscr{A}_{M, 2}(\tau), V_{\tau}\right)$ given by

$$
\operatorname{Res}_{\tau}(Q: \mu: a: x)(\psi)=-\operatorname{Res}_{\lambda=-\mu}\left(a^{-\lambda-\mu} E(Q: \psi: \lambda)(x)\right) .
$$

Proof. First, assume that $\Phi: \mathfrak{a}_{\mathfrak{q} \mathbb{C}}^{*} \rightarrow \mathbb{C}$ is a meromorphic function. Then it is readily verified that

$$
\begin{aligned}
\operatorname{Res}_{\lambda=\mu} a^{\lambda} \overline{\Phi(-\bar{\lambda})} & =a^{\mu} \operatorname{Res}_{z=0} \overline{a^{\bar{z} \omega} \Phi(-\mu-\bar{z} \omega)} \\
& =-a^{\mu} \overline{\operatorname{Res}_{z=0} a^{-z \omega} \Phi(-\mu+z \omega)} \\
& =-a^{\mu} \overline{\operatorname{Res}_{\lambda=-\mu} a^{-\lambda-\mu} \Phi(\lambda)} .
\end{aligned}
$$

By using conjugate linearity of the pairing $C_{c}^{\infty}(G / H: \tau) \times C^{\infty}(G / H: \tau) \rightarrow \mathbb{C}$ in the second factor, it now follows that the expression on the left-hand side of (7.14) equals

$$
\operatorname{Res}_{\lambda=\mu} a^{\lambda}\langle\phi, E(Q: \psi:-\bar{\lambda})\rangle=-a^{\mu}\left\langle\phi, \operatorname{Res}_{\lambda=-\mu} a^{-\lambda-\mu} E(Q: \psi: \lambda)\right\rangle .
$$

The latter expression is equal to the right-hand side of (7.14). It is clear that $\operatorname{Res}_{\tau}(Q: \mu)$ is a function in $C^{\infty}\left(A_{\mathfrak{q}} \times G / H\right) \otimes V_{\tau} \otimes \mathscr{A}_{M, 2}(\tau)^{*}$, which is $\tau$-spherical in the second variable.

The following result will be a useful tool for understanding the nature of the residues. We will use the notation $P_{d}\left(\mathfrak{a}_{\mathfrak{q}}\right)$ for the space of polynomial functions $\mathfrak{a}_{\mathfrak{q} \mathbb{C}}^{*} \rightarrow \mathbb{C}$ of degree at most $d$, and $P_{d}\left(A_{\mathfrak{q}}\right)$ for the space of functions $A_{\mathfrak{q}} \rightarrow \mathbb{C}$ of the form $a \mapsto p(\log a)$, with $p \in P_{d}\left(\mathfrak{a}_{\mathfrak{q}}\right)$.

Lemma 7.10. Let $\mu \in S_{Q, \tau}$ and $\psi \in \mathscr{A}_{M, 2}(\tau)$. Then the following assertions hold.

(a) Let $\phi$ be a holomorphic function defined on a neighborhood of $\mu$ such that the $C^{\infty}(G / H: \tau)$-valued function $\lambda \mapsto \phi(\lambda) E(Q: \psi: \lambda)$ is holomorphic at $\mu$. Then for every $u \in S\left(\mathfrak{a}_{\mathfrak{q}}^{*}\right)$,

$$
\left.\partial_{u}\right|_{\lambda=\mu} \phi(\lambda) E(Q: \psi: \lambda)
$$

is a $\mathbb{D}(G / H)$-finite function in $C^{\infty}(G / H: \tau)$.

(b) Let $\varphi$ be a meromorphic function in a neighborhood of $\mu$. Then

$$
\operatorname{Res}_{\lambda=\mu} \varphi(\lambda) E(Q: \psi: \lambda)
$$

is a $\mathbb{D}(G / H)$-finite function in $C^{\infty}(G / H: \tau)$. 
Proof. Assertion (a) follows by applying the argument of the proof of vdBS99, Lemma 6.3].

For (b) we may fix a non-trivial polynomial function $q$ such that $\phi:=q(\lambda) \varphi$ is holomorphic at $\mu$ and satisfies the hypothesis of (a). Then there exists $u \in S\left(\mathfrak{a}_{\mathfrak{q}}^{*}\right)$ such that (7.16) equals (7.15).

Lemma 7.11. Let $\mu \in S_{Q, \tau}$ and let $d \geq 0$ be the pole order of $\lambda \mapsto E(Q: \lambda)$ at $-\mu$. Then there exists a finite dimensional subspace $\mathscr{V} \subseteq C^{\infty}(G / H: \tau)$, consisting of $\mathbb{D}(G / H)$-finite functions, such that

$$
\operatorname{Res}_{\tau}(Q: \mu) \in P_{d-1}\left(A_{\mathfrak{q}}\right) \otimes \mathscr{V} \otimes \mathscr{A}_{M, 2}(\tau)^{*} .
$$

Proof. There exists a non-zero polynomial function $q$ on $\mathfrak{a}_{\mathfrak{q} \mathbb{C}}^{*}$ of degree $d$ such that the $C^{\infty}(G / H)^{K} \otimes \mathscr{A}_{M, 2}(\tau)^{*}$-valued meromorphic function $\lambda \mapsto q(\lambda) E(Q: \lambda)$ is regular at $-\mu$. It follows that there exists a $u \in S\left(\mathfrak{a}_{\mathfrak{q}}^{*}\right)$ of order at most $d-1$ such that

$$
\operatorname{Res}_{\tau}(Q: \mu: a: x) \psi=\left.\partial_{u}\right|_{\lambda=-\mu} a^{-\lambda-\mu} q(\lambda) E(Q: \psi: \lambda)(x)
$$

for all $a \in A_{\mathfrak{q}}, x \in G / H$ and $\psi \in \mathscr{A}_{M, 2}(\tau)$. By application of the Leibniz rule we infer that there exist finitely many $u_{1}, \ldots, u_{k} \in S\left(\mathfrak{a}_{\mathfrak{q}}^{*}\right)$ and $p_{1}, \ldots, p_{k} \in P_{d-1}\left(\mathfrak{a}_{\mathfrak{q}}\right)$, such that

$$
\operatorname{Res}_{\tau}(Q: \mu: a: \cdot)=\left.\sum_{j=1}^{k} p_{j}(\log a) \partial_{u_{j}}\right|_{\lambda=-\mu} q(\lambda) E(Q: \lambda)
$$

for all $a \in A_{\mathfrak{q}}$. The assertion now readily follows by application of Lemma 7.10 ,

Next, we will need the following version of Lemma 7.8.

Corollary 7.12. Let $\phi \in C_{c}(G / H: \tau)$. Then, for every $\psi \in \mathscr{A}_{M, 2}(\tau)$,

$$
\left\langle\mathcal{H}_{Q, \tau} \phi(a), \psi\right\rangle=\left\langle\mathcal{I}_{Q, \tau} \phi(a), \psi\right\rangle+\sum_{\mu \in S_{Q, \tau}} a^{\mu}\left\langle\phi, \operatorname{Res}_{\tau}(Q: \mu: a: \cdot) \psi\right\rangle .
$$

Proof. This follows from combining Lemma 7.8 with (7.14).

7.3. h-compatible parabolic subgroups. The residual terms in (7.17) will turn out to have a special relation to representations of the discrete series if we select the parabolic subgroup $Q$ so that its positive system $\Sigma(Q)$ satisfies a certain geometric restriction.

Although we will only apply the definitions and results of the present subsection to symmetric spaces of split rank 1 , everything in this subsection is in fact valid for symmetric spaces of higher split rank as well.

Definition 7.13. A parabolic subgroup $Q \in \mathscr{P}(A)$ is said to be $\mathfrak{h}$-compatible if

$$
\left\langle\alpha, \rho_{Q, \mathfrak{h}}\right\rangle \geq 0 \text { for all } \alpha \in \Sigma(Q) ;
$$

see (1.1) for the definition of $\rho_{Q, \mathfrak{h}}$. We write $\mathscr{P}_{\mathfrak{h}}(A)$ for the subset of $\mathscr{P}(A)$ consisting of all such parabolic subgroups.

\section{Lemma 7.14.}

(a) If $P_{0} \in \mathscr{P}_{\sigma}\left(A_{\mathfrak{q}}\right)$, then there exists an $\mathfrak{h}$-extreme $Q \in \mathscr{P}_{\mathfrak{h}}\left(A_{\mathfrak{q}}\right)$ such that $\Sigma(Q, \sigma \theta) \subseteq \Sigma\left(P_{0}\right)$.

(b) If $Q \in \mathscr{P}_{\mathfrak{h}}(A)$, then $\Sigma(Q, \sigma \theta) \perp \rho_{Q, \mathfrak{h}}$.

(c) If $P, Q \in \mathscr{P}(A)$ and $P \succeq Q$, then $P \in \mathscr{P}_{\mathfrak{h}}(A) \Rightarrow Q \in \mathscr{P}_{\mathfrak{h}}(A)$. 
Proof. We start with (a). Let $P_{0} \in \mathscr{P}_{\sigma}\left(A_{\mathfrak{q}}\right)$. We fix $X$ in the associated positive chamber $\mathfrak{a}_{\mathfrak{q}}^{*+}\left(P_{0}\right)$ and select a positive system $\Sigma_{\mathfrak{h}}^{+}$for the root system $\Sigma \cap \mathfrak{a}_{\mathfrak{h}}^{*}$. Put

$$
\rho_{\mathfrak{h}}:=\frac{1}{2} \sum_{\alpha \in \Sigma_{\mathfrak{h}}^{+}} m_{\alpha} \alpha .
$$

Then $\rho_{\mathfrak{h}}$ belongs to the positive chamber for $\Sigma_{\mathfrak{h}}^{+}$in $\mathfrak{a}_{\mathfrak{h}}^{*}$. There exists $Y \in \mathfrak{a}_{\mathfrak{h}}$ such that for all $\alpha \in \Sigma$,

$$
\left\langle\alpha, \rho_{\mathfrak{h}}\right\rangle>0 \Rightarrow \alpha(Y)>0 .
$$

Replacing $Y$ by a small perturbation if necessary, we may in addition assume that $Y$ belongs to the set $\mathfrak{a}_{\mathfrak{h}}^{\text {reg }}$ of elements $Z \in \mathfrak{a}_{\mathfrak{h}}$ such that for all $\alpha \in \Sigma$ we have $\alpha(Z)=\left.0 \Rightarrow \alpha\right|_{\mathfrak{a}_{\mathfrak{h}}}=0$.

We fix $\epsilon>0$ sufficiently close to zero so that for all $\alpha \in \Sigma$,

$$
\alpha(Y)>0 \Rightarrow \alpha(Y+\epsilon X)>0,
$$

and so that $Y+\epsilon X$ is a regular element in $\mathfrak{a}$. Let $Q \in \mathscr{P}(A)$ be the unique parabolic subgroup such that $Y+\epsilon X$ belongs to the positive chamber $\mathfrak{a}^{+}(Q)$. We claim that $Q$ satisfies the requirements. To see this, we start with the observation that for $\alpha \in \Sigma \backslash \mathfrak{a}_{\mathfrak{q}}^{*}$ we have $\alpha(Y) \neq 0$, so that

$$
\operatorname{sign} \alpha(Y)=\operatorname{sign} \alpha(Y+\epsilon X) .
$$

For such $\alpha$ it follows by application of (7.20) to both $\alpha$ and $\sigma \alpha$ that

$$
\operatorname{sign} \sigma \alpha(Y+\epsilon X)=\operatorname{sign} \alpha(Y+\epsilon X) .
$$

Thus, we see that for $\alpha \in \Sigma \backslash \mathfrak{a}_{\mathfrak{q}}^{*}$ we have $\alpha \in \Sigma(Q)$ if and only if $\sigma \alpha \in \Sigma(Q)$. Thus, $\Sigma(Q) \backslash \mathfrak{a}_{\mathfrak{q}}^{*}=\Sigma(Q, \sigma)$ and we infer that $Q$ is $\mathfrak{h}$-extreme.

If $\alpha \in \Sigma(Q) \cap \mathfrak{a}_{\mathfrak{q}}^{*}$, then

$$
\epsilon \alpha(X)=\alpha(Y+\epsilon X)>0
$$

and we obtain that $\alpha \in \Sigma\left(P_{0}\right)$. Hence, $\Sigma(Q, \sigma \theta)=\Sigma(Q) \cap \mathfrak{a}_{\mathfrak{q}}^{*} \subseteq \Sigma\left(P_{0}\right)$.

Next, assume that $\alpha \in \Sigma$ satisfies $\left\langle\alpha, \rho_{\mathfrak{h}}\right\rangle \neq 0$. Then in view of (7.18) and (7.19) we have

$$
\operatorname{sign}\left\langle\alpha, \rho_{\mathfrak{h}}\right\rangle=\operatorname{sign} \alpha(Y)=\operatorname{sign} \alpha(Y+\epsilon X) .
$$

In particular, the above is valid for $\alpha \in \Sigma_{\mathfrak{h}}^{+}$. From this we see that $\Sigma_{\mathfrak{h}}^{+}=\Sigma(Q) \cap \mathfrak{a}_{\mathfrak{h}}^{*}$, so that $\rho_{Q, \mathfrak{h}}=\rho_{\mathfrak{h}}$.

For the proof of (a), it remains to be shown that $Q$ is $\mathfrak{h}$-compatible. Let $\alpha \in$ $\Sigma(Q)$. If $\left\langle\alpha, \rho_{Q \mathfrak{h}}\right\rangle \neq 0$, then it follows from (7.21) that $\left\langle\alpha, \rho_{Q, \mathfrak{h}}\right\rangle=\left\langle\alpha, \rho_{\mathfrak{h}}\right\rangle>0$. This establishes (a).

We turn to (b). Let $\alpha \in \Sigma(Q, \sigma \theta)$. Then $\left\langle\alpha, \rho_{Q, \mathfrak{h}}\right\rangle \geq 0$. On the other hand, $\sigma \theta \alpha \in \Sigma(Q)$ hence

$$
0 \leq\left\langle\sigma \theta \alpha, \rho_{Q, \mathfrak{h}}\right\rangle=-\left\langle\alpha, \rho_{Q, \mathfrak{h}}\right\rangle
$$

and we see that $\alpha \perp \rho_{Q, \mathfrak{h}}$.

Finally, assume that $P, Q$ satisfy the hypotheses of (c) and that $P \in \mathscr{P}_{\mathfrak{h}}(A)$. From $P \succeq Q$ it follows that $\Sigma(P) \cap \mathfrak{a}_{\mathfrak{h}}^{*}=\Sigma(Q) \cap \mathfrak{a}_{\mathfrak{h}}^{*}$, hence $\rho_{P, \mathfrak{h}}=\rho_{Q, \mathfrak{h}}$. Since $P$ is $\mathfrak{h}$-compatible and since (b) holds, we see that every root $\alpha$ from the set

$$
\Psi:=\Sigma(P) \cup-\Sigma(P, \sigma \theta)
$$


satisfies $\left\langle\alpha, \rho_{Q, \mathfrak{h}}\right\rangle \geq 0$. We will finish the proof by showing that $\Sigma(Q) \subseteq \Psi$. Let $\alpha \in$ $\Sigma(Q)$. Then either $\alpha \in \Sigma(Q, \sigma \theta)$ or $\alpha \in \Sigma(Q, \sigma)$. In the first case, $\alpha \in \Sigma(P, \sigma \theta) \subseteq \Psi$. Thus, assume $\alpha \in \Sigma(Q, \sigma)$. If $\alpha \in \Sigma(P, \sigma)$, then $\alpha \in \Psi$. Thus, it remains to consider the case that $\alpha \notin \Sigma(P, \sigma)$. Since $-\alpha \in \Sigma(P, \sigma)$ would imply $-\alpha \in \Sigma(Q, \sigma) \subseteq \Sigma(Q)$, contradiction, both $\alpha$ and $-\alpha$ do not belong to $\Sigma(P, \sigma)$. It follows that one of them must belong to $\Sigma(P, \sigma \theta)$, hence $\alpha \in \Psi$.

Example 7.15 (Group case). We use notation as in Example 3.2. Every element of $\mathscr{P}\left({ }^{\prime} A \times{ }^{\prime} A\right)$ is of the form $P={ }^{\prime} P \times{ }^{\prime} Q$, where ' $P$ and ' $Q$ are minimal parabolic subgroups containing ' $A$. All roots are non-zero on $\mathfrak{a}_{\mathfrak{q}}$, hence $\rho_{P, \mathfrak{h}}=0$. Each of these parabolic subgroups is therefore $\mathfrak{h}$-compatible.

The importance of the notion of $\mathfrak{h}$-compatibility comes from the following result, which implies that if $Q \in \mathscr{P}_{\mathfrak{h}}(A)$, then certain singularities of $E(Q: \cdot)$ are caused by singularities of $E^{\circ}\left(\bar{P}_{0}: \cdot\right)$; see also Lemma 5.4.

Proposition 7.16. Let $P_{0}$ be a minimal $\sigma \theta$-stable parabolic subgroup containing A. Let $Q \in \mathscr{P}_{\mathfrak{h}}(A)$ and assume that $\Sigma(Q: \sigma \theta) \subseteq \Sigma\left(P_{0}\right)$. Then $C_{\bar{P}_{0} \mid Q}(1: \cdot)$ is holomorphic on $-\mathfrak{a}_{\mathfrak{q}}^{*+}\left(P_{0}\right)+i \mathfrak{a}_{\mathfrak{q}}^{*}$.

Proof. Assume that $\lambda \in-\mathfrak{a}_{\mathfrak{q}}^{*+}\left(P_{0}\right)+i \mathfrak{a}_{\mathfrak{q}}^{*}$. Then $\operatorname{Re}\langle\lambda, \alpha\rangle<0$ for all $\alpha \in \Sigma\left(P_{0}\right)$ and $\left\langle\rho_{Q, \mathfrak{h}}, \alpha\right\rangle \geq 0$ for all $\alpha \in \Sigma(Q)$. Hence,

$$
\operatorname{Re}\left\langle-\lambda+\rho_{Q, \mathfrak{h}}, \alpha\right\rangle>0 \text { for all } \alpha \in \Sigma\left(P_{0}\right) \cap \Sigma(Q) .
$$

The result now follows by application of Proposition 5.5(b).

We end this section with a result about $\mathscr{W}$-conjugates of $\mathfrak{h}$-compatible parabolic subgroups.

Lemma 7.17. If $Q \in \mathscr{P}_{\mathfrak{h}}(A)$, then $Q^{v} \in \mathscr{P}_{\mathfrak{h}}(A)$ for every $v \in N_{K}\left(\mathfrak{a}_{\mathfrak{q}}\right) \cap N_{K}\left(\mathfrak{a}_{\mathfrak{h}}\right)$. In particular, this is valid for $v \in \mathscr{W}$.

Proof. Since $v$ normalizes both $\mathfrak{a}$ and $\mathfrak{a}_{\mathfrak{h}}$, it follows that

$$
\Sigma\left(Q^{v}\right) \cap \mathfrak{a}_{\mathfrak{h}}^{*}=v^{-1} \cdot\left(\Sigma(Q) \cap \mathfrak{a}_{\mathfrak{h}}^{*}\right),
$$

hence $\rho_{Q^{v}, \mathfrak{h}}=v^{-1} \cdot \rho_{Q, \mathfrak{h}}$. The lemma now follows from the fact that $v$ acts isometrically on $\mathfrak{a}^{*}$.

7.4. Residues for the trivial $K$-type. In this subsection we retain the following.

Assumption. $G / H$ is of split rank 1 .

Our goal is to analyze the residues of Eisenstein integrals $E(Q: \lambda)$ as introduced in Lemma 7.9, for $Q \in \mathscr{P}_{\mathfrak{h}}(A)$ and for $\left(\tau, V_{\tau}\right)$ equal to the trivial representation $(1, \mathbb{C})$ of $K$. To emphasize that $\tau=1$, we denote the associated Eisenstein integrals with $E_{1}(Q: \lambda)$; see also (5.5).

As before, we assume that $P_{0}$ is a minimal $\sigma \theta$-stable parabolic subgroup containing $A$ and such that $\Sigma(Q, \sigma \theta) \subseteq \Sigma\left(P_{0}\right)$.

If $\pi$ is a discrete series representation for $G / H$, we agree to write $\mathscr{C}(G / H)_{\pi}$ for the closed subspace of $\mathscr{C}(G / H)$ spanned by left $K$-finite and right $H$-fixed generalized matrix coefficients of $\pi$. 
Proposition 7.18. Assume that $Q \in \mathscr{P}_{\mathfrak{h}}(A)$. There exist finitely many spherical discrete series representations $\pi_{1}, \ldots, \pi_{k}$ such that for all $\mu \in S_{Q, 1}$, all $\psi \in \mathscr{A}_{M, 2}(1)$ and all $a \in A_{\mathfrak{q}}$,

$$
\operatorname{Res}_{1}(Q: \mu: a: \cdot)(\psi) \in \bigoplus_{i=1}^{k} \mathscr{C}(G / H)_{\pi_{i}} .
$$

We will prove this proposition through a series of results. First, we need to prepare for these. Let $W\left(\mathfrak{a}_{\mathfrak{q}}\right)$ be the Weyl group of the root system of $\mathfrak{a}_{\mathfrak{q}}$ in $\mathfrak{g}$. Then $W\left(\mathfrak{a}_{\mathfrak{q}}\right)=\{-1,1\}$, since $\operatorname{dim}\left(\mathfrak{a}_{\mathfrak{q}}\right)=1$ by assumption. The map

$$
\mathscr{A}_{M, 2}(1) \longrightarrow \mathbb{C}^{\mathscr{W}}, \quad \psi \mapsto\left(\psi_{w}(e): w \in \mathscr{W}\right)
$$

is a linear isomorphism via which we shall identify the indicated spaces.

Let $\psi \in \mathscr{A}_{M, 2}(1)$. Then from vdBK14, Thm. 8.13] it follows that, for $R \in$ $\mathscr{P}_{\sigma}\left(A_{\mathfrak{q}}\right), w \in \mathscr{W}, b \in A_{\mathfrak{q}}^{+}(R)$ and generic $\lambda \in \mathfrak{a}_{\mathfrak{q} \mathbb{C}}^{*}$,

$$
E_{1}(Q: \psi: \lambda)(b w)=\sum_{s \in\{ \pm 1\}} \Phi_{R, w}(s \lambda: b)\left[C_{R \mid Q}(s: \lambda) \psi\right]_{w}(e) .
$$

Here $\Phi_{R, w}(\lambda, \cdot)$, for generic $\lambda \in \mathfrak{a}_{\mathfrak{q C}}^{*}$, is a certain function on $A_{\mathrm{q}}^{+}(R)$ defined as in vdBS97a, Thm. 11.1], for $\tau=1$. We recall that the functions are related by the equations

$$
\Phi_{R, w}(\lambda, a)=\Phi_{w^{-1} R w, 1}\left(w^{-1} \lambda, w^{-1} a w\right)
$$

for generic $\lambda \in \mathfrak{a}_{\mathfrak{q} \mathbb{C}}^{*}$ and all $a \in A_{\mathrm{q}}^{+}(R)$; see vdBS97a, Lemma 10.3]. It follows from these relations and $\mathrm{vdBS97a}$, Eqn. (15)] that the function $\Phi_{R, w}(\lambda, \cdot)$ for generic $\lambda \in \mathfrak{a}_{\mathfrak{q} \mathbb{C}}^{*}$ has a converging series expansion of the form

$$
\Phi_{R, w}(\lambda, a)=a^{\lambda-\rho_{R}} \sum_{k \geq 0} \Gamma_{R, w, k}(\lambda) a^{-k \alpha} \quad\left(a \in A_{\mathrm{q}}^{+}\left(\bar{P}_{0}\right)\right),
$$

where $\alpha$ denotes the unique indivisible root in $\Sigma\left(R, \mathfrak{a}_{\mathfrak{q}}\right)$. The coefficients $\Gamma_{R, w, k}$, for $k \in \mathbb{N}$, are meromorphic functions on $\mathfrak{a}_{\mathfrak{q} \mathbb{C}}^{*}$, which are uniquely determined by the following conditions (see vdBS97a, Prop. 5.2, Eqn. (19)]), taking into account that $\tau\left(L_{\alpha}^{ \pm}\right)=0$ and $\gamma=0$, because $\tau$ is trivial.

(1) $\Gamma_{R, w, 0}(\lambda)=1$ for all $\lambda \in \mathfrak{a}_{\mathfrak{q} \mathbb{C}}^{*}$.

(2) The function $\lambda \mapsto\left(\prod_{l=1}^{k}\langle 2 \lambda-l \alpha, \alpha\rangle\right) \cdot \Gamma_{R, w, k}(\lambda)$ is entirely holomorphic on $\mathfrak{a}_{\mathfrak{q} \mathbb{C}}^{*}$.

In the proof ahead, we will need the following additional properties of the functions $\Phi_{R, w}$ and their expansions.

Lemma 7.19. Let $\Omega$ be a bounded open subset of $\mathfrak{a}_{\mathfrak{q}}^{*}$. Then there exists a polynomial function $q \in \Pi_{\Sigma, \mathbb{R}}\left(\mathfrak{a}_{\mathfrak{q}}^{*}\right)$ (see (5.17)), such that the following assertions are valid.

(a) For every $k \geq 0$ the function $q \Gamma_{R, w, k}$ is holomorphic on $\Omega+i \mathfrak{a}_{\mathfrak{q}}$.

(b) The power series

$$
\sum_{k \geq 0} q(\lambda) \Gamma_{R, w, k}(\lambda) z^{k}
$$

converges absolutely on $D=\{z \in \mathbb{C}:|z|<1\}$, locally uniformly in $(\lambda, z) \in\left(\Omega+i \mathfrak{a}_{\mathfrak{q}}^{*}\right) \times D$. In particular, it defines a holomorphic function on the mentioned set. 
(c) The assignment $a \mapsto q(\lambda) \Phi_{R, w}(\lambda: a)$ defines a smooth function on $A_{\mathfrak{q}}$, depending holomorphically on $\lambda \in \Omega \times i \mathfrak{a}_{\mathfrak{q}}^{*}$.

(d) For all $\lambda \in \Omega+i \mathfrak{a}_{\mathfrak{q}}^{*}$ and $a \in A_{\mathfrak{q}}^{+}(R)$,

$$
q(\lambda) \Phi_{R, w}(\lambda, a)=q(\lambda) a^{\lambda-\rho_{R}} \sum_{k \geq 0} \Gamma_{R, w, k}(\lambda) a^{-k \alpha} .
$$

Proof. In view of the relations (7.23), we may assume that $w=1$. By boundedness of $\Omega$ there exists $n \geq 1$ such that $\langle 2 \lambda-l \alpha, \alpha\rangle \neq 0$ for all $l>n$ and $\lambda \in \Omega+i \mathfrak{a}_{\mathfrak{q}}^{*}$. In view of conditions (1) and (2) above, we see that the polynomial

$$
q(\lambda)=\prod_{l=1}^{n}\langle 2 \lambda-l \alpha, \alpha\rangle
$$

satisfies the requirements of (a).

In view of (a), it follows from the estimate for the coefficients given in vdBS97a, Thm. 7.4], that for every compact subset $U \subseteq \Omega+i \mathfrak{a}_{\mathfrak{q}}^{*}$ there exists a constant $C>0$ and an integer $\kappa>0$ such that

$$
\left|q(\lambda) \Gamma_{R, 1, k}(\lambda)\right| \leq C(1+k)^{\kappa} \quad(\lambda \in U) .
$$

From this, (b) follows readily. Finally, (c) and (d) are immediate consequences of (a), (b) and (7.24).

Based on the lemma, we can prove another preparatory result.

Proposition 7.20. Let $\mu \in \mathfrak{a}_{\mathfrak{q}}^{*+}\left(P_{0}\right)$. Then for every holomorphic function $f$ : $\mathfrak{a}_{\mathfrak{q} \mathbb{C}}^{*} \rightarrow \mathbb{C}$ and every $\psi \in \mathscr{A}_{M, 2}(\tau)$ the function

$$
\operatorname{Res}_{\lambda=-\mu} f(\lambda) E_{1}(Q: \psi: \lambda): G / H \rightarrow \mathbb{C}
$$

is $\mathbb{D}(G / H)$-finite and contained in $\mathscr{C}(G / H)^{K}$.

Remark 7.21. Actually the result is valid for any holomorphic function $f$ defined on an open neighborhood of $-\mu$, but we will not need this.

Proof of Proposition 7.18, Let $F$ be the function (7.25). It follows from Lemma 7.10(b) that $F$ belongs to $C^{\infty}(G / H)^{K}$ and is $\mathbb{D}(G / H)$-finite.

We will complete the proof by showing that $F$ belongs to the Schwartz space. Fix $w \in \mathscr{W}$, then by $\operatorname{vdB} 87 \mathrm{a}$, Thm. 6.4] it suffices to establish, for any $\varepsilon>0$, the existence of a constant $C=C_{\varepsilon}>0$ such that

$$
|F(b w)| \leq C b^{-\mu+\varepsilon \alpha-\rho_{\bar{P}_{0}}} \quad\left(b \in A_{\mathrm{q}}^{+}\left(\bar{P}_{0}\right)\right) .
$$

Taking $R=\bar{P}_{0}$ we obtain that, for $w \in \mathscr{W}$, for $b \in A_{\mathfrak{q}}^{+}\left(\bar{P}_{0}\right)$ and generic $\lambda \in \mathfrak{a}_{\mathfrak{q} c}^{*}$,

$$
E_{1}(Q: \psi: \lambda)(b w)=\sum_{s \in\{ \pm 1\}} \Phi_{\bar{P}_{0}, w}(s \lambda: b)\left[C_{\bar{P}_{0} \mid Q}(s: \lambda) \psi\right]_{w}(e) .
$$

Here $C_{\bar{P}_{0} \mid Q}(1: \cdot)$ is holomorphic at the element $-\mu \in \mathfrak{a}_{\mathfrak{q}}^{*+}\left(\bar{P}_{0}\right)$.

For $k \geq 1$ we define the polynomial function $q_{k}:=\langle\cdot+\mu, \alpha\rangle^{k}$ on $\mathfrak{a}_{\mathfrak{q C}}^{*}$. Let $\Omega$ be a bounded open neighborhood of $-\mu$ in $\mathfrak{a}_{\mathfrak{q}}^{*}$. Then we may select an integer $k_{1} \geq 0$ such that the polynomial $q_{k_{1}}$ satisfies all properties of Lemma 7.19 for $R=\bar{P}_{0}$ and all $w \in \mathscr{W}$. In addition we may fix $k_{2} \geq 0$ such that the function

$$
\lambda \mapsto q_{k_{2}}(\lambda) C_{\bar{P}_{0} \mid Q}(s: \lambda)
$$


is holomorphic on an open neighborhood $U$ of $-\mu$ in $\Omega+i \mathfrak{a}_{\mathfrak{q}}^{*}$ for each $s \in\{ \pm 1\}$. Put $q=q_{k_{1}} q_{k_{2}}$, then it follows that $q(\lambda) E_{1}(Q: \psi: \lambda)(b w)$ is smooth in $(\lambda, b) \in$ $U \times A_{\mathrm{q}}^{+}\left(\bar{P}_{0}\right)$ and holomorphic in the first of these variables.

For each $s \in\{ \pm 1\}$, we define the disjoint decomposition

$$
\mathbb{N}=\mathcal{N}(s,+) \cup \mathcal{N}(s,-)
$$

by

$$
k \in \mathcal{N}(s,+) \Longleftrightarrow\langle-2 s \mu-k \alpha, \alpha\rangle>0 .
$$

Accordingly, we put

$$
\Phi_{\bar{P}_{0}, w}^{ \pm}(s \lambda, b)=b^{-\rho_{\bar{P}_{0}}} \sum_{k \in \mathcal{N}(s, \pm)} \Gamma_{\bar{P}_{0}, w, k}(s \lambda) b^{s \lambda-k \alpha} .
$$

Then

$$
\Phi_{\bar{P}_{0}, w}(s \lambda, b)=\Phi_{\bar{P}_{0}, w}^{+}(s \lambda, b)+\Phi_{\bar{P}_{0}, w}^{-}(s \lambda, b) .
$$

It is easily seen that $\mathcal{N}(s,+)$ is finite and without gaps in $\mathbb{N}$. Furthermore, we may shrink $U$ to arrange that $\langle 2 s \lambda-k \alpha, \alpha\rangle>0$ for all $s= \pm 1, k \in \mathcal{N}(s,+)$ and $\lambda \in U$. In view of property (2) below (7.24) this implies that the function

$$
\Phi_{\bar{P}_{0}, w}^{+}(s \lambda, b)
$$

is holomorphic in $\lambda \in U$, for each $s= \pm 1$. Furthermore, if $s=-1$, then $\langle-s \mu, \alpha\rangle<$ 0 and we see that $\mathcal{N}(-1,+)=\emptyset$ so that in fact

$$
\Phi_{\bar{P}_{0}, w}^{+}(-\lambda, b)=0 \text {. }
$$

We agree to write

$$
\left.\Psi^{ \pm}(\lambda, b)\right)=\sum_{s \in\{ \pm 1\}} \Phi_{\bar{P}_{0}, w}^{ \pm}(s \lambda: b)\left[C_{\bar{P}_{0} \mid Q}(s: \lambda) \psi\right]_{w}(e)
$$

so that

$$
E_{1}(Q: \psi: \lambda)(b w)=\Psi^{+}(\lambda, b)+\Psi^{-}(\lambda, b) .
$$

Taking into account that $C_{\bar{P}_{0} \mid Q}(1: \cdot)$ is holomorphic at the point $-\mu$, it follows from the above that $\Psi^{+}(\lambda, b)$ is holomorphic at $\lambda=-\mu$, so that

$$
\operatorname{Res}_{\lambda=-\mu}\left[f(\lambda) \Psi^{+}(\lambda, b)\right]=0 \quad\left(b \in A_{\mathrm{q}}^{+}\left(P_{0}\right)\right) .
$$

We infer that

$$
F(b w)=\operatorname{Res}_{\lambda=-\mu} f(\lambda) \Psi^{-}(\lambda, b) .
$$

We will now derive an estimate for $f(\lambda) q(\lambda) \Psi^{-}(\lambda, b)$ by looking at the exponents of the series expansion. If $s=1$, then it follows that for $k \in \mathcal{N}(s,-)$ we have

$$
\langle-s \mu-k \alpha, \alpha\rangle \leq 0+\langle\mu, \alpha\rangle<0 \text {. }
$$

Shrinking $U$ we may arrange that for all $\lambda \in U$ and all $k \in \mathcal{N}(s,-)$,

$$
\operatorname{Re}\langle s \lambda-k \alpha, \alpha\rangle \leq\langle\mu+\epsilon \alpha, \alpha\rangle .
$$

As described in Lemma 7.19 the series for $q_{1}(\lambda) \Phi_{\bar{P}_{0}, w}(s \lambda: b)$ is essentially a power series in $b^{-\alpha}$, with holomorphic dependence on $\lambda$. Hence, we may shrink $U$ to arrange that there exists a constant $C>0$ such that for $\lambda \in U$ and $b \in A_{\mathfrak{q}}$ with $b^{\alpha} \geq 2$ we have

$$
\left|q_{1}(\lambda) \Phi_{\bar{P}_{0}, w}^{-}(s \lambda, b)\right| \leq C b^{-\mu-\rho_{\bar{P}_{0}}+\epsilon \alpha} .
$$


On the other hand, if $s=-1$, then for all $k \in \mathbb{N}$ we have

$$
\langle-s \mu-k \alpha, \alpha\rangle \leq\langle\mu, \alpha\rangle<0
$$

and we see that by shrinking $U$ even further if necessary, we may arrange that (17.29) is valid for the present choice of $s$, all $k \in \mathbb{N}$ and all $\lambda \in U$. This leads to the estimate (7.30) for $s=-1$. From the estimates obtained, combined with the holomorphy of the function (7.27), for $s= \pm 1$, we infer that there exists a constant $C_{1}>0$ such that

$$
\left|f(\lambda) q(\lambda) \Psi^{-}(\lambda, b)\right| \leq C_{1} b^{-\rho_{\bar{P}_{0}}+(-\mu+\epsilon \alpha)}
$$

for $\lambda \in U$ and $b^{\alpha} \geq 2$. Using the integral formula for the residue in (7.28) and taking into account that $q(\lambda)^{-1}$ is bounded on a circle around $-\mu$ in $U$, we infer that there exists $C>0$ such that $(7.26)$ is valid for all $b \in A_{\mathfrak{q}}$ with $b^{\alpha} \geq 2$. Since $F$ is continuous, a similar estimate holds for all $b \in A_{\mathrm{q}}^{+}\left(\bar{P}_{0}\right)$.

We finally come to the proof of Proposition 7.18 .

Proof of Proposition 7.18. Let $\mu \in S_{Q, 1}$. It follows from Lemma 7.11 that there exists an integer $d \geq 0$ and a finite dimensional subspace $\mathscr{V} \subseteq C^{\infty}(G / H)^{K}$, consisting of $\mathbb{D}(G / H)$-finite functions, such that

$$
\operatorname{Res}_{1}(Q: \mu) \psi \in P_{d}\left(A_{\mathfrak{q}}\right) \otimes \mathscr{V}
$$

for all $\psi \in \mathscr{A}_{M, 2}(1)$. On the other hand, it follows by application of Proposition 7.20 that $\operatorname{Res}_{1}(Q: \mu: a: \cdot)$ is a $\mathbb{D}(G / H)$-finite function in $\mathscr{C}(G / H)^{K}$ for every $a \in A_{\mathfrak{q}}$. Hence, (17.31) is valid with $\mathscr{V}$ a finite dimensional subspace of $\mathscr{C}(G / H)^{K}$, consisting of $\mathbb{D}(G / H)$-finite functions, which are therefore in particular $Z(\mathfrak{g})$-finite.

It now follows that the $(\mathfrak{g}, K)$-span of $\mathscr{V}$ in $\mathscr{C}(G / H)$ is a $(\mathfrak{g}, K)$-module of finite length in view of a well-known result of Harish-Chandra; see Var77, p. 312, Thm. 12] and Wal88, p. 112, Thm. 4.2.1].) The closure of this span in $L^{2}(G / H)$ is therefore a finite direct sum of irreducible subrepresentations. Since $\mathscr{V}$ consists of left $K$-invariant functions, each of these irreducible subrepresentations is spherical. The result follows.

Remark 7.22. The proof of Proposition 7.18 relies heavily on the assumption that $\mathfrak{a}_{\mathfrak{q}}$ is one-dimensional, which makes it possible to analyse which exponents vanish from the expansion involved, by taking residues.

7.5. Convergence for symmetric spaces of split rank one. In this subsection we retain the following.

Assumption. $G / H$ is of split rank one.

Theorem 7.23. Let $Q \in \mathscr{P}_{\mathfrak{h}}(A)$. Then $\mathcal{H}_{Q}$ has a unique extension to a continuous linear map $\mathscr{C}(G / H) \rightarrow C^{\infty}\left(L / H_{L}\right)$. Moreover, for every $\phi \in \mathscr{C}(G / H)$,

$$
\mathcal{H}_{Q} \phi(l)=\delta_{Q}(l) \int_{N_{Q} / H_{N_{Q}}} \phi(l n) d n \quad(l \in L)
$$

with absolutely convergent integrals. Furthermore, the Radon transform $\mathcal{R}_{Q}$ has a unique extension to a continuous linear map $\mathscr{C}(G / H) \rightarrow C^{\infty}\left(G / N_{Q}\right)$ and for every $\phi \in \mathscr{C}(G / H)$,

$$
\mathcal{R}_{Q} \phi(g)=\int_{N_{Q} / H_{N_{Q}}} \phi(g n) d n \quad(g \in G),
$$

with absolutely convergent integrals. 
Proof. As in (7.22) we identify $\mathscr{A}_{M, 2}(1) \simeq \mathbb{C}^{\mathscr{W}}$. Let $\psi_{0} \in \mathscr{A}_{M, 2}(1)$ be the unique element determined by $\left(\psi_{0}\right)_{w}=\delta_{1 w}$ for $w \in \mathscr{W}$.

By Lemma 6.2 we see that, for $\phi \in C_{c}^{\infty}(G / H)^{K}$,

$$
\left\langle\mathcal{H}_{Q, 1} \phi(a), \psi_{0}\right\rangle=\mathcal{H}_{Q} \phi(a) \quad\left(a \in A_{\mathfrak{q}}\right) .
$$

It follows from Proposition 7.18 that the functions $\operatorname{Res}_{1}(Q: \mu: a: \cdot)\left(\psi_{0}\right)$, for $\mu \in S_{Q, \tau}$, belong to $\mathscr{C}(G / H)$ for every $a \in A_{\mathfrak{q}}$. Furthermore, by Lemma 7.11 , these functions depend polynomially on $a$. If we combine this with Proposition 7.2, we infer that the expression on the right-hand side of (7.17) is well-defined for $\phi \in \mathscr{C}(G / H: 1)=\mathscr{C}(G / H)^{K}$ and depends linear continuously on it with values in

$$
C^{\infty}\left(A_{\mathfrak{q}}\right)=C^{\infty}\left(L / H_{L}\right)^{M} .
$$

It follows that the restriction of $\mathcal{H}_{Q}$ to $C_{c}^{\infty}(G / H)^{K}$ extends to a continuous linear map from $\mathscr{C}(G / H)^{K}$ to $C^{\infty}\left(L / H_{L}\right)^{M}$. The theorem now follows by application of Proposition 4.6

Remark 7.24. For the hyperbolic spaces $\mathrm{SO}(p, q+1)_{e} / \mathrm{SO}(p, q)_{e}$, this result is due to AFJS12.

In the following we assume more generally that $\left(\tau, V_{\tau}\right)$ is a finite dimensional unitary representation of $K$.

Corollary 7.25. Let $Q \in \mathscr{P}_{\mathfrak{h}}(A)$. Then $\mathcal{H}_{Q, \tau}$ extends to a continuous linear map

$$
\mathcal{H}_{Q, \tau}: \mathscr{C}(G / H: \tau) \rightarrow C^{\infty}\left(A_{\mathfrak{q}}\right) \otimes \mathscr{A}_{M, 2}(\tau) .
$$

Moreover, (6.1) and (6.2) are valid for every $\phi \in \mathscr{C}(G / H: \tau)$ with the extensions of $\mathcal{H}_{Q, \tau}$ and $\mathcal{H}_{Q^{v}}$ to the associated Schwartz spaces; the appearing integrals are absolutely convergent.

Proof. Since $C_{c}^{\infty}(G / H: \tau)$ is dense in $\mathscr{C}(G / H: \tau)$, this follows immediately from combining Theorem 7.23 with Lemma 7.17.

By Proposition 7.2 the map $\mathcal{I}_{Q, \tau}$ extends to a continuous linear map from $\mathscr{C}(G / H: \tau)$ to $C^{\infty}\left(A_{\mathfrak{q}}\right) \otimes \mathscr{A}_{M, 2}(\tau)$ as well. Hence, it follows from equation (7.17) that for every $a \in A_{\mathfrak{q}}$ and $\psi \in \mathscr{A}_{M, 2}(\tau)$,

$$
\sum_{\mu \in S_{Q, \tau}} a^{\mu} \operatorname{Res}_{\tau}(Q: \mu: a: \cdot) \psi
$$

is a smooth function defining a tempered distribution. In particular, writing

$$
C_{\text {temp }}^{\infty}(G / H):=C^{\infty}(G / H) \cap \mathscr{C}^{\prime}(G / H),
$$

we obtain the following.

Corollary 7.26. Let $Q \in \mathscr{P}_{\mathfrak{h}}(A)$ and let $\mu \in S_{Q, \tau}$. Then

$$
\operatorname{Res}_{\tau}(Q: \mu) \in P\left(A_{\mathfrak{q}}\right) \otimes C_{\text {temp }}^{\infty}(G / H) \otimes \operatorname{Hom}\left(\mathscr{A}_{M, 2}(\tau), V_{\tau}\right) .
$$

We now observe that Proposition [7.2, Corollary 7.25 and Corollary 7.26 imply the following result.

Corollary 7.27. Let $Q \in \mathscr{P}_{\mathfrak{h}}(A)$. Then equation (7.17) holds for every $\phi \in$ $\mathscr{C}(G / H: \tau), \psi \in \mathscr{A}_{M, 2}(\tau)$, and $a \in A_{\mathfrak{q}}$. 


\section{Cusp Forms And Discrete SERIES REPRESEntations}

A well-known result of Harish-Chandra asserts for the case of the group that the closed span in the Schwartz space of the bi- $K$-finite matrix coefficients of the representations from the discrete series equals the space of so-called cusp forms. (See [HC66], [HC70, Thm. 10], [HC75, Sect. 18,27] and [Var77, Thm. 16.4.17].) In the present section we generalize this result for the case of split rank one.

8.1. The kernel of $\mathcal{I}_{Q, \tau}$. In the present subsection we do not make any assumption on the dimension of $\mathfrak{a}_{\mathfrak{q}}$. Let $\left(\tau, V_{\tau}\right)$ be a finite dimensional unitary representation of $K$ as before.

Theorem 8.1. Let $Q \in \mathscr{P}(A)$ and let $P_{0} \in \mathscr{P}_{\sigma}(A)$. Then the following are equal as subspaces of $\mathscr{C}(G / H: \tau)$,

$$
\operatorname{ker}\left(\mathcal{F}_{\bar{P}_{0}}\right)=\operatorname{ker}\left(\mathcal{I}_{Q, \tau}\right)
$$

Proof. Let $\phi \in \mathscr{C}(G / H: \tau)$. Then the definition of $\mathcal{K}_{Q, \tau} \phi$ in (7.5) is meaningful and the equality (7.7) is valid. If $\mathcal{F}_{\bar{P}_{0}} \phi=0$, then it follows from (7.4) that $\mathcal{K}_{Q, \tau} \phi=0$, which in turn implies that $\mathcal{I}_{Q, \tau} \phi=0$. This shows that the space on the left-hand side of (8.1) is contained in the space on the right-hand side.

For the converse inclusion, let $p_{h}, u$ and $v$ be as in the proof of Proposition 7.2 , Let $D$ be the bi-invariant differential operator on $A_{\mathfrak{q}}$ which satisfies

$$
\mathcal{F}_{A_{\mathfrak{q}}}\left(D \delta_{e}\right)=p_{h},
$$

with $\delta_{e}$ the Dirac measure at the point $e$ on $A_{\mathfrak{q}}$. Then

$$
\mathcal{F}_{A_{\mathfrak{q}}}\left(D\left(\mathcal{I}_{Q, \tau} \phi\right)\right)=p_{h} \mathcal{F}_{A_{\mathfrak{q}}}\left(\mathcal{I}_{Q, \tau} \phi\right)=p_{h} \mathcal{F}_{A_{\mathfrak{q}}}\left(\mathcal{K}_{Q, \tau} \phi\right) v .
$$

If $\xi \in \mathfrak{a}_{\mathfrak{q}}^{*}$ is a real linear functional, then by looking at real and imaginary parts, we see that $|\langle\lambda, \xi\rangle| \leq|\langle\lambda+\epsilon \nu, \xi\rangle|$ for all $\lambda \in i \mathfrak{a}_{\mathfrak{q}}^{*}, \epsilon>0$ and $\nu \in \mathfrak{a}_{\mathfrak{q}}^{*}$. This implies that

$$
\left|\frac{p_{h}(\lambda)}{p_{h}(\lambda+\epsilon \nu)}\right| \leq 1 \quad\left(\lambda \in \mathfrak{a}_{\mathfrak{q}}^{*}\right) .
$$

By a straightforward application of the Lebesgue dominated convergence theorem it now follows that $p_{h} v=1$, and we infer that

$$
D\left(\mathcal{I}_{Q, \tau} \phi\right)=\mathcal{K}_{Q, \tau} \phi
$$

Now let $\phi \in \mathscr{C}(G / H: \tau)$ and assume that $\mathcal{I}_{Q, \tau} \phi=0$. Then $\mathcal{K}_{Q, \tau} \phi=0$ and it follows from (17.5) that $\left[p_{h} \mathfrak{F}_{Q, \tau}\right] \phi=0$. In view of (7.4) this implies that for generic $\lambda \in i \mathfrak{a}_{\mathfrak{q}}^{*}$

$$
p_{h}(\lambda) C_{\bar{P}_{0}: Q}(1:-\bar{\lambda})^{*} \mathcal{F}_{\bar{P}_{0}}(\phi)(\lambda)=0 .
$$

Since $\mathcal{F}_{\bar{P}_{0}} \phi$ is smooth by vdBS97b, Cor. 4, p. 573] and $C_{\bar{P}_{0}: Q}(1:-\bar{\lambda})$ is invertible for generic $\lambda \in i \mathfrak{a}_{\mathfrak{q}}^{*}$, by $\operatorname{vdBK14}$, Thm. 8.13], it follows that $\mathcal{F}_{\bar{P}_{0}} \phi=0$.

Let $\mathscr{C}_{\mathrm{mc}}(G / H: \tau)$ be the closed subspace of $\mathscr{C}(G / H: \tau)$ corresponding to the most continuous part of the spectrum. By vdBS97c, Cor. 17.2 and Prop. 17.3] the kernel of $\mathcal{F}_{\bar{P}_{0}}$ is equal to the orthocomplement (with respect to the $L^{2}$-inner product) in $\mathscr{C}(G / H: \tau)$ of $\mathscr{C}_{\mathrm{mc}}(G / H: \tau)$. Theorem 8.1 therefore implies that

$$
\mathscr{C}_{\text {mc }}(G / H: \tau) \cap \operatorname{ker}\left(\mathcal{I}_{Q, \tau}\right)=\{0\} .
$$

Let $\mathscr{C}_{\mathrm{ds}}(G / H: \tau)$ be the closed span in $\mathscr{C}(G / H: \tau)$ of the $K$-finite generalized matrix coefficients of the representations from the discrete series for $G / H$. Then 
the space $\mathscr{C}_{\mathrm{ds}}(G / H: \tau)$ is contained in the orthocomplement of $\mathscr{C}_{\mathrm{mc}}(G / H: \tau)$ and therefore in the kernel of $\mathcal{I}_{Q, \tau}$ for every $Q \in \mathscr{P}(A)$.

Corollary 8.2. If $\operatorname{dim}\left(\mathfrak{a}_{\mathfrak{q}}\right)=1$, then $\operatorname{ker}\left(\mathcal{I}_{Q, \tau}\right)=\mathscr{C}_{\mathrm{ds}}(G / H: \tau)$.

Proof. This follows from the above discussion combined with vdBS97c, Prop. 17.7]

8.2. Residues for arbitrary $K$-types. In this subsection we will work under the following.

Assumption. $G / H$ is of split rank one.

Let $\left(\tau, V_{\tau}\right)$ be a finite dimensional unitary representation of $K$ and let $Q \in$ $\mathscr{P}_{\mathfrak{h}}(A)$. Let $P_{0} \in \mathscr{P}_{\sigma}\left(A_{\mathfrak{q}}\right)$ be such that $\Sigma(Q, \sigma \theta) \subseteq \Sigma\left(P_{0}\right)$. We recall that the singular set $S_{Q, \tau}$ is a finite subset of $\mathfrak{a}_{\mathfrak{q}}^{*+}\left(P_{0}\right)$; see Lemma 7.7. The first main result of this subsection is that the residues appearing in (7.17) are $L^{2}$-perpendicular to the part of the Schwartz space corresponding to the most continuous part of the Plancherel formula.

Theorem 8.3. Let $\mu \in S_{Q, \tau}$. For every $\phi \in \mathscr{C}_{\mathrm{mc}}(G / H: \tau), \psi \in \mathscr{A}_{M, 2}(\tau)$ and $a \in A_{\mathfrak{q}}$,

$$
\left\langle\phi \operatorname{Res}_{\tau}(Q: \mu: a: \cdot) \psi\right\rangle=0 .
$$

This result will be proved through a series of partial results. Let $P_{0}$ be a parabolic subgroup as above and let $P_{0}=M_{0} A_{0} N_{0}$ be its Langlands decomposition. Via symmetrization of the associated quadratic form, the symmetric $G$-equivariant bilinear form $B$ of (1.4) gives rise to the Casimir operator $\Omega$ in the center of $U(\mathfrak{g})$. The image $\Delta_{G / H} \in \mathbb{D}(G / H)$ of $\Omega$ under the map (6.12) will be called the Laplacian of $G / H$. Likewise, the restriction of $B$ to $\mathfrak{a}_{\mathfrak{q}} \times \mathfrak{a}_{\mathfrak{q}}$ gives rise to the Laplacian $\Delta_{A_{\mathfrak{q}}}$ of $A_{\mathfrak{q}}$. The Casimir $\Omega_{\mathfrak{m}_{0}}$ of $\mathfrak{m}_{0} \cap \mathfrak{k}$ is defined by applying symmetrization to the restriction of $B$ to $\mathfrak{m}_{0} \cap \mathfrak{k}$.

Lemma 8.4. Let $\Delta_{G / H}, \Delta_{A_{\mathfrak{q}}}$ and $\Omega_{\mathfrak{m}_{0}}$ be as above. Then for $\phi \in \mathscr{C}(G / H: \tau)$,

$$
\mathcal{H}_{Q, \tau}\left(\Delta_{G / H} \phi\right)=\left(\tau\left(\Omega_{\mathfrak{m}_{0}}\right)+\Delta_{A_{\mathfrak{q}}}-\left\langle\rho_{P_{0}}, \rho_{P_{0}}\right\rangle\right) \mathcal{H}_{Q, \tau} \phi .
$$

Proof. First, we observe that (6.15) is valid for Schwartz functions $\phi \in \mathscr{C}(G / H)$, in view of Corollary 7.25 and density of $C_{c}^{\infty}(G / H: \tau)$ in $\mathscr{C}(G / H: \tau)$.

Next, from vdBS97a, Lemma 5.3] it readily follows that

$$
\mu\left(\Delta_{G / H}: \tau\right)=\tau\left(\Omega_{\mathfrak{m}_{0}}\right)+\Delta_{A_{\mathfrak{q}}}-\left\langle\rho_{P_{0}}, \rho_{P_{0}}\right\rangle .
$$

Now apply (6.15).

We write $\widehat{M}_{0 H}$ for the set of $\xi \in \widehat{M}_{0}$ such that $V(\xi) \neq 0$. For $\xi \in \widehat{M}_{0 H}$ let $\mathscr{A}_{M, 2}(\tau)_{\xi}$ be the image of $C(K: \xi: \tau) \otimes \overline{V(\xi)}$ under the map $T \mapsto \psi_{T}$ defined by (5.7). Then $\mathscr{A}_{M, 2}(\tau)$ decomposes into a finite direct sum of orthogonal subspaces

$$
\mathscr{A}_{M, 2}(\tau)=\bigoplus_{\xi \in \widehat{M}_{0 H}} \mathscr{A}_{M, 2}(\tau)_{\xi} .
$$

The action of $N_{K}\left(\mathfrak{a}_{\mathfrak{q}}\right)$ on $M_{0}$ by conjugation naturally induces a (left) action of the Weyl group $W\left(\mathfrak{a}_{\mathfrak{q}}\right)=N_{K}\left(\mathfrak{a}_{\mathfrak{q}}\right) / Z_{K}\left(\mathfrak{a}_{\mathfrak{q}}\right)$ on $\widehat{M}_{0 H}$. We agree to use $W$ as an 
abbreviation for $W\left(\mathfrak{a}_{\mathfrak{q}}\right)$ in the rest of this subsection. Accordingly, for $\xi \in \widehat{M}_{0 H}$ we define

$$
\mathscr{A}_{M, 2}(\tau)_{W \cdot \xi}:=\bigoplus_{w \in W} \mathscr{A}_{M, 2}(\tau)_{w \xi} \cdot
$$

Accordingly, we obtain the orthogonal decomposition

$$
\mathscr{A}_{M, 2}(\tau)=\bigoplus_{W \cdot \xi \in W \backslash \widehat{M}_{0}} \mathscr{A}_{M, 2}(\tau)_{W \cdot \xi}
$$

We define the normalized $C$-function $C_{\bar{P}_{0} \mid \bar{P}_{0}}^{\circ}(s: \cdot)$, for $s \in W$, to be the $\operatorname{End}\left(\mathscr{A}_{M, 2}(\tau)\right)$-valued meromorphic function on $\mathfrak{a}_{\mathfrak{q} \mathbb{C}}^{*}$ given by

$$
C_{\bar{P}_{0} \mid \bar{P}_{0}}^{\circ}(s: \lambda)=C_{\bar{P}_{0} \mid \bar{P}_{0}}(s: \lambda) C_{\bar{P}_{0} \mid \bar{P}_{0}}(1: \lambda)^{-1}
$$

for generic $\lambda \in \mathfrak{a}_{\mathfrak{q C}}^{*}$; see vdBS97b, Eqn. (55)]. Let $\gamma$ be the (unitary) representation of $W$ in $L^{2}\left(i \mathfrak{a}_{\mathfrak{q}}^{*}\right) \otimes \mathscr{A}_{M, 2}(\tau)$ defined as in vdBS97c, Eqn. (16.1)], with $\bar{P}_{0}$ in place of $P$. It is given by

$$
[\gamma(s) f](\lambda)=C_{\bar{P}_{0} \mid \bar{P}_{0}}^{\circ}\left(s^{-1}: \lambda\right)^{-1} f\left(s^{-1} \lambda\right)
$$

for $s \in W, f \in L^{2}\left(i \mathfrak{a}_{\mathfrak{q}}^{*}\right) \otimes \mathscr{A}_{M, 2}(\tau)$ and $\lambda \in i \mathfrak{a}_{\mathfrak{q}}^{*}$.

Lemma 8.5. Let $\xi \in \widehat{M}_{0 H}$. Then the subspace

$$
\mathscr{S}\left(i \mathfrak{a}^{*}\right) \otimes \mathscr{A}_{M, 2}(\tau)_{W \cdot \xi} \subseteq L^{2}\left(i \mathfrak{a}_{\mathfrak{q}}^{*}\right) \otimes \mathscr{A}_{M, 2}(\tau)
$$

is invariant for $\gamma$.

Proof. First, the subspace $\mathscr{S}\left(i \mathfrak{a}_{\mathfrak{q}}^{*}\right) \otimes \mathscr{A}_{M, 2}(\tau)$ is $\gamma$-invariant by the argument suggested in vdBS97b, Rem. 16.3].

Next, let $f \in \mathscr{S}\left(i \mathfrak{a}_{\mathfrak{q}}^{*}\right) \otimes \mathscr{A}_{M, 2}(\tau)_{\xi}$, where $\xi \in \widehat{M}_{0 H}$. Let $s \in W$ and let $w \in N_{K}\left(\mathfrak{a}_{\mathfrak{q}}\right)$ be a representative for $s$. Then it suffices to show that

$$
[\gamma(s) f](\lambda) \in \mathscr{A}_{M, 2}(\tau)_{s \xi} \quad\left(\lambda \in i \mathfrak{a}_{\mathfrak{q}}^{*}\right) .
$$

In turn, for this it suffices to prove the claim that

$$
C_{\bar{P}_{0} \mid \bar{P}_{0}}^{\circ}\left(s^{-1}: \lambda\right) \mathscr{A}_{M, 2}(\tau)_{s \xi} \subseteq \mathscr{A}_{M, 2}(\tau)_{\xi},
$$

for any $\xi \in \widehat{M}_{0 H}$ and generic $\lambda \in i \mathfrak{a}_{\mathfrak{q}}^{*}$.

By (8.4) and vdBS97b, Lemma 7],

$$
\begin{aligned}
C_{\bar{P}_{0} \mid \bar{P}_{0}}^{\circ}\left(s^{-1}: \lambda\right) & =C_{\bar{P}_{0} \mid \bar{P}_{0}}\left(s^{-1}: \lambda\right) \circ C_{\bar{P}_{0} \mid \bar{P}_{0}}(1: \lambda)^{-1} \\
& =\mathscr{L}\left(s^{-1}\right) \circ C_{s \bar{P}_{0} s^{-1} \mid \bar{P}_{0}}(1: \lambda) \circ C_{\bar{P}_{0} \mid \bar{P}_{0}}(1: \lambda)^{-1},
\end{aligned}
$$

for generic $\lambda \in \mathfrak{a}_{\mathfrak{q} \mathbb{C}}^{*}$. Here $\mathscr{L}\left(s^{-1}\right)$ is given by vdBS97b, Eqn. (65)]

$$
\mathscr{L}\left(s^{-1}\right) \psi_{T}=\psi_{\left[L\left(w^{-1}\right) \otimes L\left(s \xi, w^{-1}\right)\right] T} \quad(T \in C(K: s \xi: \tau) \otimes \bar{V}(s \xi)),
$$

where $L\left(w^{-1}\right): C^{\infty}(K: s \xi: \tau) \rightarrow C^{\infty}(K: \xi: \tau)$ and $L\left(s \xi, w^{-1}\right): \bar{V}(s \xi) \rightarrow \bar{V}(\xi)$ are linear maps. Accordingly, we see that $\mathscr{L}\left(s^{-1}\right)$ maps $\mathscr{A}_{M, 2}(\tau)_{s \xi}$ to $\mathscr{A}_{M, 2}(\tau)_{\xi}$.

From vdBS97b, Prop. 1] and the definition of the $B$-matrix in vdB88, Prop. 6.1] it follows that $C_{s \bar{P}_{0} s^{-1} \mid \bar{P}_{0}}(1: \lambda) \circ C_{\bar{P}_{0} \mid \bar{P}_{0}}(1: \lambda)^{-1}$ preserves $\mathscr{A}_{M, 2}(\tau)_{s \xi}$. Thus, 88.5) follows. 
By vdBS97c, Cor. 17.4], the Fourier transform $\mathcal{F}_{\bar{P}_{0}}$ defines a topological linear isomorphism from $\mathscr{C}_{\text {mc }}(G / H: \tau)$ onto $\left[\mathscr{S}\left(i \mathfrak{a}_{\mathfrak{q}}^{*}\right) \otimes \mathscr{A}_{M, 2}(\tau)\right]^{W}$. For $\xi \in \widehat{M}_{0 H}$ we define

$$
\mathscr{C}_{\mathrm{mc}}(G / H: \tau)_{W \cdot \xi}:=\mathcal{F}_{\bar{P}_{0}}^{-1}\left(\left[\mathscr{S}\left(i \mathfrak{a}^{*}\right) \otimes \mathscr{A}_{M, 2}(\tau)_{W \cdot \xi}\right]^{W}\right) .
$$

Then in view of $(8.3)$ it follows that

$$
\mathscr{C}_{\mathrm{mc}}(G / H: \tau)=\bigoplus_{W \cdot \xi \in W \backslash \widehat{M}_{0 H}} \mathscr{C}_{\mathrm{mc}}(G / H: \tau)_{W \cdot \xi}
$$

with only finitely many non-zero summands.

Lemma 8.6. Let $\xi \in \widehat{M}_{0 H}$. If $\phi \in \mathscr{C}_{\mathrm{mc}}(G / H: \tau)_{W \cdot \xi}$, then

$$
\mathcal{H}_{Q, \tau} \phi \in C^{\infty}\left(A_{\mathfrak{q}}\right) \otimes \mathscr{A}_{M, 2}(\tau)_{W \cdot \xi} \cdot
$$

Proof. Let $\phi \in \mathscr{C}_{\text {mc }}(G / H: \tau)_{W \cdot \xi}$. Then $\mathcal{F}_{\bar{P}_{0}} \phi \in \mathscr{S}\left(i \mathfrak{a}_{\mathfrak{q}}^{*}\right) \otimes \mathscr{A}_{M, 2}(\tau)_{W \cdot \xi}$. The lemma now follows by application of Propositions 5.5, 5.7, and 6.4.

Lemma 8.7. Let $\xi \in \widehat{M}_{0 H}$. Then there exists a scalar $c_{\xi} \in \mathbb{R}$ such that $\tau\left(\Omega_{\mathfrak{m}_{0}}\right)$ acts on $\mathscr{A}_{M, 2}(\tau)_{W \cdot \xi}$ by the scalar $c_{\xi}$.

Proof. From vdBK14, Cor. 4.4] it follows that the restriction of $\xi$ to $M_{0} \cap K$ is irreducible. Hence, $\xi\left(\Omega_{\mathfrak{m}_{0}}\right)$ acts by a real scalar $c_{\xi}$ on $\mathscr{H}_{\xi}$.

Let $s \in W$. Then $s$ has a representative $w \in N_{K}\left(\mathfrak{a}_{\mathfrak{q}}\right)$. As $\operatorname{Ad}(w)$ preserves the restriction of the bilinear form $B$ to $\mathfrak{k} \cap \mathfrak{m}_{0}$, it follows that $\operatorname{Ad}(w) \Omega_{\mathfrak{m}_{0}}=\Omega_{\mathfrak{m}_{0}}$. This implies that $s \xi\left(\Omega_{\mathfrak{m}_{0}}\right)$ acts by the scalar $c_{\xi}$ on $\mathscr{H}_{\xi}$. We infer that

$$
c_{s \xi}=c_{\xi} \quad(s \in W) .
$$

Let $s \in W, f \in C(K: s \xi: \tau)$ and let $\eta \in \bar{V}(s \xi)$. Then, with notation as in (5.7),

$$
\begin{aligned}
\left(\tau\left(\Omega_{\mathfrak{m}_{0}}\right) \psi_{f \otimes \eta}\right)(m) & =\left\langle\left(1 \otimes \tau\left(\Omega_{\mathfrak{m}_{0}}\right)\right) f(m), \eta\right\rangle \\
& =\left\langle\left(s \xi\left(\Omega_{\mathfrak{m}_{0}}\right) \otimes 1\right) f(m), \eta\right\rangle \\
& =c_{\xi} \psi_{f \otimes \eta}(m) .
\end{aligned}
$$

The assertion now follows.

For $\xi \in \widehat{M}_{0 H}$ we define the differential operator $D_{\xi} \in \mathbb{D}(G / H)$ by

$$
D_{\xi}:=\prod_{\mu \in S_{Q, \tau}}\left(\Delta_{G / H}-c_{\xi}+\left\langle\rho_{P_{0}}, \rho_{P_{0}}\right\rangle-\langle\mu, \mu\rangle\right)^{m_{\mu}},
$$

where $m_{\mu}-1 \geq 0$ is the degree of the $C^{\infty}(G / H) \otimes \operatorname{Hom}\left(\mathscr{A}_{M, 2}(\tau), V_{\tau}\right)$-valued polynomial function $a \mapsto \operatorname{Res}_{\tau}(Q: \mu: a: \cdot)$.

Lemma 8.8. Let $\xi \in \widehat{M}_{0 H}$. Then for every $\phi \in \mathscr{C}_{\mathrm{mc}}(G / H: \tau)_{W \cdot \xi}$,

$$
\mathcal{H}_{Q, \tau}\left(D_{\xi} \phi\right)=\left(\prod_{\mu \in S_{Q, \tau}}\left(\Delta_{A_{\mathfrak{q}}}-\langle\mu, \mu\rangle\right)^{m_{\mu}}\right) \mathcal{H}_{Q, \tau} \phi .
$$

Proof. The lemma follows directly from Lemma 8.4. Lemma 8.6 and Lemma 8.7

Lemma 8.9. Let $\xi \in \widehat{M}_{0 H}$. Then for every $\phi \in \mathscr{C}_{\mathrm{mc}}(G / H: \tau)_{W \cdot \xi}$ there exists a $\chi \in \mathscr{C}_{\mathrm{mc}}(G / H: \tau)_{W \cdot \xi}$ such that

$$
D_{\xi} \chi=\phi
$$


Proof. For $\lambda \in i \mathfrak{a}_{\mathfrak{q}}^{*}$ we define $e_{\lambda}: A_{\mathfrak{q}} \rightarrow \mathbb{C}, a \mapsto a^{\lambda}$. Moreover, for $D \in \mathbb{D}(G / H)$ we define $\mu(D: \tau: \lambda) \in \operatorname{End}\left(\mathscr{A}_{M, 2}(\tau)\right)$ by

$$
\mu(D: \tau: \lambda) \psi:=\left(\mu(D: \tau)\left(e^{\lambda} \otimes \psi\right)\right)(e) \quad\left(\psi \in \mathscr{A}_{M, 2}(\tau)\right)
$$

here $\mu(D: \tau)$ is the $\operatorname{End}\left(\mathscr{A}_{M, 2}(\tau)\right)$-valued differential operator on $A_{\mathfrak{q}}$ defined in (6.14).

Then for all $D \in \mathbb{D}(G / H), \phi \in \mathscr{C}(G / H: \tau)$ and $\lambda \in i \mathfrak{a}_{\mathfrak{q}}^{*}$, we have

$$
\mathcal{F}_{\bar{P}_{0}}(D \phi)(\lambda)=\mu(D: \tau: \lambda) \mathcal{F}_{\bar{P}_{0}} \phi(\lambda) ;
$$

see vdBS97c, Lemma 6.2]. In particular, it follows that

$$
\mathcal{F}_{\bar{P}_{0}}\left(D_{\xi} \phi\right)(\lambda)=\prod_{\mu \in S_{Q, \tau}}(\langle\lambda, \lambda\rangle-\langle\mu, \mu\rangle)^{m_{\mu}} \mathcal{F}_{\bar{P}_{0}} \phi(\lambda) .
$$

Note that $\langle\lambda, \lambda\rangle-\langle\mu, \mu\rangle \neq 0$ for $\lambda \in i \mathfrak{a}_{\mathfrak{q}}^{*}$ and $\mu \in S_{Q, \tau} \subseteq \mathfrak{a}_{\mathfrak{q}}^{*} \backslash\{0\}$.

Now let $\phi \in \mathscr{C}_{\mathrm{mc}}(G / H: \tau)_{W \cdot \xi}$. Then the function $f: i \mathfrak{a}_{\mathfrak{q}}^{*} \rightarrow \mathscr{A}_{M, 2}(\tau)$ defined by

$$
f(\lambda)=\prod_{\mu \in S_{Q, \tau}}(\langle\lambda, \lambda\rangle-\langle\mu, \mu\rangle)^{-m_{\mu}} \mathcal{F}_{\bar{P}_{0}} \phi(\lambda)
$$

belongs to the space $\left(S\left(i \mathfrak{a}_{\mathfrak{q}}^{*}\right) \otimes \mathscr{A}_{M, 2}(\tau)_{W \cdot \xi}\right)^{W}$. The assertion of the lemma now follows with $\chi=\mathcal{F}_{\bar{P}_{0}}^{-1} f$.

Proof of Theorem 8.3. Since $\mathscr{C}_{\mathrm{mc}}(G / H: \tau)$ decomposes as a finite direct sum (8.6), it suffices to prove the assertion for $\phi \in \mathscr{C}_{\mathrm{mc}}(G / H: \tau)_{W \cdot \xi}$. Let $\phi$ be such a function and let $\chi$ be as in Lemma 8.9 . Then by Lemma 8.8

$$
\mathcal{H}_{Q, \tau} \phi=\left(\prod_{\mu \in S_{Q, \tau}}\left(\Delta_{A_{\mathfrak{q}}}-\langle\mu, \mu\rangle\right)^{m_{\mu}}\right) \mathcal{H}_{Q, \tau} \chi .
$$

Since $a \mapsto\left\langle\chi, \operatorname{Res}_{\tau}(Q: \mu: a: \cdot) \psi\right\rangle$ is a polynomial function of degree $m_{\mu}-1$, it follows that

$$
\left(\Delta_{A_{\mathfrak{q}}}-\langle\mu, \mu\rangle\right)^{m_{\mu}} a^{\mu}\left\langle\chi, \operatorname{Res}_{\tau}(Q: \mu: a: \cdot) \psi\right\rangle=0,
$$

hence

$$
\mathcal{H}_{Q, \tau} \phi=\left(\prod_{\mu \in S_{Q, \tau}}\left(\Delta_{A_{\mathfrak{q}}}-\langle\mu, \mu\rangle\right)^{m_{\mu}}\right) \mathcal{I}_{Q, \tau} \chi .
$$

In particular, $\mathcal{H}_{Q, \tau} \phi$ belongs to $C_{\text {temp }}^{\infty}\left(A_{\mathfrak{q}}\right) \otimes \mathscr{A}_{M, 2}(\tau)$. Since $\mathcal{I}_{Q, \tau} \phi$ also belongs to this space, by Proposition 7.2 , we infer that

$$
a \mapsto \sum_{\mu \in S_{Q, \tau}} a^{\mu}\left\langle\operatorname{Res}_{\tau}(Q: \mu: a: \cdot), \phi\right\rangle
$$

belongs to this space. Since the latter sum is also an exponential polynomial function with non-zero exponents on $A_{\mathfrak{q}}$, with values in $\mathscr{A}_{M, 2}(\tau)$, it must be zero and we finally conclude (8.2).

We now come to the second theorem of this subsection, which asserts that in fact the residual functions from Corollary 7.26 are constant as functions of the variable from $A_{\mathfrak{q}}$. From Corollary 7.26 we recall that for $\mu \in S_{Q, \tau}$, the function $\operatorname{Res}_{\tau}(Q, \mu)$ belongs to $P\left(A_{\mathfrak{q}}\right) \otimes C_{\text {temp }}^{\infty}(G / H: \tau) \otimes \mathscr{A}_{M, 2}(\tau)^{*}$. 
Theorem 8.10. Let $\mu \in S_{Q, \tau}$.

(a) The function $\operatorname{Res}_{\tau}(Q: \mu)$ is constant with respect to the variable from $A_{\mathfrak{q}}$ and belongs to $\mathscr{C}_{\mathrm{ds}}(G / H: \tau) \otimes \mathscr{A}_{M, 2}(\tau)^{*}$.

(b) $E(Q:-\cdot)$ has a pole of order 1 at $\mu$ as a $C^{\infty}(G / H) \otimes \operatorname{Hom}\left(\mathscr{A}_{M, 2}(\tau), V_{\tau}\right)$ valued meromorphic function on $\mathfrak{a}_{\mathfrak{q}, \mathbb{C}}^{*}$.

Proof. By vdBS97c, Prop. 17.7]

$$
\mathscr{C}(G / H: \tau)=\mathscr{C}_{\mathrm{mc}}(G / H: \tau) \oplus \mathscr{C}_{\mathrm{ds}}(G / H: \tau)
$$

as an orthogonal direct sum. By Corollary 7.26 the finite dimensional space

$$
\mathscr{V}=\operatorname{span}\left\{\operatorname{Res}_{\tau}(Q: \mu: a) \psi: \mu \in S_{Q, \tau}, a \in A_{\mathfrak{q}}, \psi \in \mathscr{A}_{M, 2}(\tau)\right\}
$$

is contained in $C_{\text {temp }}^{\infty}(G / H: \tau) \subseteq \mathscr{C}^{\prime}(G / H: \tau)$. Since $\mathscr{C}_{\mathrm{ds}}(G / H: \tau)$ is finite dimensional (see [OM84] and vdBS05, Lemma $12.6 \&$ Rem. 12.7]), there exists for every $\chi \in \mathscr{V}$ a function $\vartheta \in \mathscr{C}_{\mathrm{ds}}(G / H: \tau)$ such that

$$
\langle\phi, \chi\rangle=\langle\phi, \vartheta\rangle
$$

for every $\phi \in \mathscr{C}_{\mathrm{ds}}(G / H: \tau)$. By Theorem 8.3 the space $\mathscr{C}_{\mathrm{mc}}(G / H: \tau)$ is perpendicular to $\mathscr{V}$. Hence, (8.7) is valid for every $\phi \in \mathscr{C}(G / H: \tau)$, and we conclude that $\chi=\vartheta$. This proves that $\mathscr{V} \subseteq \mathscr{C}_{\mathrm{ds}}(G / H: \tau)$.

The space $\mathscr{C}_{\text {ds }}(G / H: \tau)$ decomposes as a finite direct sum

$$
\mathscr{C}_{\mathrm{ds}}(G / H: \tau)=\bigoplus_{\pi} \mathscr{C}(G / H: \tau)_{\pi},
$$

where $\pi$ runs over the representations of the discrete series and $\mathscr{C}(G / H: \tau)_{\pi}$ is spanned by left $\tau$-spherical and right $H$-fixed generalized matrix coefficients of $\pi$.

Let $\pi$ be a discrete series representation, $\phi \in \mathscr{C}(G / H: \tau)_{\pi}$ and $\psi \in \mathscr{A}_{M, 2}(\tau)$. We will establish (a) by showing that $\left\langle\phi, \operatorname{Res}_{\tau}(Q: \mu: a: \cdot) \psi\right\rangle$ is independent of $a \in A_{\mathfrak{q}}$. Since (8.3) is a finite direct sum, we may assume that $\psi \in \mathscr{A}_{M, 2}(\tau)_{W \cdot \xi}$ for a representation $\xi \in \widehat{M}_{0 H}$.

By Corollary 7.27 and Corollary 8.2 ,

$$
\left\langle\mathcal{H}_{Q, \tau} \phi(a), \psi\right\rangle=\sum_{\mu \in S_{Q, \tau}} a^{\mu}\left\langle\phi, \operatorname{Res}_{\tau}(Q: \mu: a: \cdot) \psi\right\rangle \quad\left(a \in A_{\mathfrak{q}}\right) .
$$

Since $\pi$ is irreducible, $\phi$ is an eigenfunction for $\Delta_{G / H}=R_{\Omega}$. Let $c$ be its eigenvalue. Then by Lemma 8.4

$$
\left(\tau\left(\Omega_{\mathfrak{m}_{0}}\right)+\Delta_{A_{\mathfrak{q}}}-\left\langle\rho_{P_{0}}, \rho_{P_{0}}\right\rangle-c\right) \mathcal{H}_{Q, \tau} \phi=0 .
$$

Since $\tau\left(\Omega_{\mathfrak{m}_{0}}\right)$ is symmetric, we have for $\chi \in \mathscr{A}_{M, 2}(\tau)$,

$$
\left\langle\tau\left(\Omega_{\mathfrak{m}_{0}}\right) \chi, \psi\right\rangle=\left\langle\chi, \tau\left(\Omega_{\mathfrak{m}_{0}}\right) \psi\right\rangle=c_{\xi}\langle\chi, \psi\rangle
$$

We thus see that

$$
\left(\Delta_{A_{\mathfrak{q}}}+c_{\xi}-\left\langle\rho_{P_{0}}, \rho_{P_{0}}\right\rangle-c\right)\left\langle\mathcal{H}_{Q, \tau} \phi, \psi\right\rangle=0
$$

The solutions to this differential equation are either polynomial functions (in case $c_{\xi}-\left\langle\rho_{P_{0}}, \rho_{P_{0}}\right\rangle-c=0$ ) or a sum of exponential functions (in case $c_{\xi}-\left\langle\rho_{P_{0}}, \rho_{P_{0}}\right\rangle-c \neq$ $0)$. By comparing with (8.8) we see that $\left\langle\mathcal{H}_{Q, \tau} \phi, \psi\right\rangle$ cannot be purely polynomial, hence must be a finite sum of exponentials functions. This establishes (a).

We turn to (b). We define the linear function $p: \mathfrak{a}_{\mathfrak{q} \mathbb{C}}^{*} \rightarrow \mathbb{C}$ by $p(\lambda)=\langle\lambda+\mu, \omega\rangle$, where $\omega$ is the unique unit vector in $\mathfrak{a}_{\mathfrak{q}}^{*}\left(P_{0}\right)$. We note that

$$
p(-\mu+z \omega)=z \quad(z \in \mathbb{C}) .
$$


The function $E(Q:-\cdot)$ has a singularity at $\mu$, by definition of the set $S_{Q, \tau}$. Reasoning by contradiction, assume that (b) is not valid. Then there exists an element $\psi \in \mathscr{A}_{M, 2}(\tau)$ and a $k \geq 1$ such that $p^{k+1} E(Q: \psi:-\cdot)$ is regular at $\mu$ and has a non-zero value at that point. Then with the notation of (17.13) and Lemma 7.9, it follows that

$$
\begin{aligned}
\operatorname{Res}_{\tau}(Q: \mu: a: x) \psi & =-\operatorname{Res}_{\lambda=-\mu} a^{-\lambda-\mu} E(Q: \psi: \lambda)(x) \\
& =-\operatorname{Res}_{z=0} a^{-z \omega} E(Q: \psi:-\mu+z \omega)(x) \\
& =-\left.\frac{d^{k}}{d z^{k}}\right|_{z=0} z^{k+1} a^{-z \omega} E(Q: \psi:-\mu+z \omega)(x) \\
& =\sum_{j=0}^{k} \omega(\log a)^{j} \chi_{j}(x)
\end{aligned}
$$

with uniquely determined functions $\chi_{j} \in C^{\infty}(G / H: \tau)$. By the Leibniz rule it follows in particular that

$$
\chi_{k}(x)=(-1)^{k+1} \omega(\log a)^{k}\left[z^{k+1} E(Q: \psi:-\mu+z \omega)\right]_{z=0} .
$$

Since

$$
\left[z^{k+1} E(Q: \psi:-\mu+z \omega)\right]_{z=0}=\left[p(\lambda) E(Q: \psi: \lambda]_{\lambda=-\mu} \neq 0\right.
$$

this implies that $\operatorname{Res}_{\tau}(Q: \mu: a: x)(\psi)$ is not constant in $a$, contradicting (a). Hence (b) is valid.

In view of Theorem 8.10 we now obtain the following version of Corollary 7.27 .

Corollary 8.11. Let $\phi \in \mathscr{C}(G / H: \tau)$. Then for all $\psi \in \mathscr{A}_{M, 2}(\tau)$ and $a \in A_{\mathfrak{q}}$,

$$
\left\langle\mathcal{H}_{Q, \tau} \phi(a), \psi\right\rangle=\left\langle\mathcal{I}_{Q, \tau} \phi(a), \psi\right\rangle+\sum_{\mu \in S_{Q, \tau}} a^{\mu}\left\langle\phi, \operatorname{Res}_{\tau}(Q, \mu) \psi\right\rangle .
$$

8.3. Cusp Forms. In this final subsection we keep working under the following.

Assumption. $G / H$ is of split rank one.

The following definition makes use of the Radon transform introduced in Definition 2.12

Definition 8.12. A function $\phi \in \mathscr{C}(G / H)$ is called a cusp form if $\mathcal{R}_{Q} \phi=0$ for every $Q \in \mathscr{P}_{\mathfrak{h}}(A)$. We write $\mathscr{C}_{\text {cusp }}(G / H)$ for the subspace of such cusp forms in $\mathscr{C}(G / H)$.

Lemma 8.13. $\mathscr{C}_{\text {cusp }}(G / H)$ is a $G$-invariant closed subspace of $\mathscr{C}(G / H)$.

Proof. This follows immediately from Theorem 7.23 and the $G$-equivariance of $\mathcal{R}_{Q}$, for every $Q \in \mathscr{P}_{\mathfrak{h}}(A)$.

Recall that a parabolic subgroup $Q \in \mathscr{P}(A)$ is said to be $\mathfrak{h}$-extreme if $\Sigma(Q, \sigma \theta)=$ $\Sigma(Q) \cap \mathfrak{a}_{\mathfrak{q}}^{*}$.

Lemma 8.14. Let $\phi \in \mathscr{C}(G / H)$. Then the following conditions are equivalent.

(a) $\phi$ is a cusp form.

(b) $\mathcal{R}_{Q} \phi=0$ for every $\mathfrak{h}$-extreme parabolic subgroup $Q \in \mathscr{P}_{\mathfrak{h}}(A)$. 
Proof. Clearly, (b) follows from (a). For the converse, assume that (b) holds. Let $P \in \mathscr{P}_{\mathfrak{h}}(A)$. There exists a $\mathfrak{h}$-extreme $Q \in \mathscr{P}(A)$ such that $P \succeq Q$; see $\mathrm{BvdB} 14$, Lemma 2.6]. By Lemma 7.14 (c) we see that $Q \in \mathscr{P}_{\mathfrak{h}}(A)$, so that $\mathcal{R}_{Q} \phi=0$. Since the integral for $\mathcal{R}_{P} \phi(g)$ is absolutely convergent, for every $g \in G$, it now follows by application of Corollary 2.4 that

$$
\mathcal{R}_{P} \phi(g)=\int_{N_{P} \cap \bar{N}_{Q}} \mathcal{R}_{Q} \phi(g n) d n=0 .
$$

Thus, (a) follows.

Remark 8.15. It follows from this result that for the class of real hyperbolic spaces $\mathrm{SO}(p, q+1)_{e} / \mathrm{SO}(p, q)_{e}$ our notion of cusp form coincides with the one introduced by AFJS12, Eqn. (5)]. Indeed, the minimal parabolic subgroup mentioned in the text following [AFJS12, Eqn. (5)] is h-extreme in our sense, and it turns out the condition of $\mathfrak{h}$-compatibility is fulfilled. In fact, it is easy to see that for this family of symmetric spaces the properties of $\mathfrak{h}$-compatibility and $\mathfrak{h}$-extremeness coincide.

Remark 8.16. Let $\vartheta$ be a finite subset of $\widehat{K}$. For a representation of $K$ on a vector space $V$, we denote by $V_{\vartheta}$ the subspace of $K$-finite vectors with isotypes contained in $\vartheta$.

Let $C(K)_{\vartheta}$ be the space of $K$-finite continuous functions on $K$, whose right $K$-types belong to $\vartheta$ and let $\tau$ denote the right regular representation of $K$ on $V_{\tau}:=C(K)_{\vartheta}$. Then the canonical map

$$
\varsigma: \mathscr{C}(G / H)_{\vartheta} \rightarrow \mathscr{C}(G / H: \tau)
$$

given by

$$
\varsigma \phi(x)(k)=\phi(k x) \quad\left(\phi \in \mathscr{C}(G / H)_{\vartheta}, k \in K, x \in G / H\right)
$$

is a linear isomorphism. Let $\phi \in \mathscr{C}(G / H)_{\vartheta}$. Then it follows from (6.2) (see Corollary (7.25), that $\mathcal{H}_{Q^{v}} \phi=0$ for every $v \in \mathscr{W}$ if and only if $\mathcal{H}_{Q, \tau}(\varsigma \phi)=0$. Hence, $\phi \in \mathscr{C}_{\text {cusp }}(G / H)$ if and only if $\mathcal{H}_{Q, \tau}(\varsigma \phi)=0$ for every $Q \in \mathscr{P}_{\mathfrak{h}}(A)$.

Example 8.17 (Group case). We use notation as in Example 3.2

Every minimal parabolic subgroup is $\mathfrak{h}$-compatible (see Example 7.15); the $\mathfrak{h}$ extreme parabolic subgroups are all of the form ' $P \times{ }^{\prime} P$ where $' P$ is a minimal parabolic subgroup of ' $G$. As explained in Example 3.2, the Radon transform $\mathcal{R}_{\backslash_{P \times} P}$ is identified with $\mathcal{R}_{\backslash P}$ under the identification $\left({ }^{\prime} G \times{ }^{\prime} G\right) / \operatorname{diag}\left({ }^{\prime} G\right)$. From Lemma 8.14 it now follows that $\phi \in \mathscr{C}(G / H)$ is a cusp form if and only if $\mathcal{R}_{\backslash} \phi=$ 0 for every minimal parabolic subgroup ' $P$ of ' $G$. Using the fact that ' $G$ acts transitively on the set of minimal parabolic subgroups of ' $G$, it is easily seen that this in turn is equivalent to the condition that

$$
\int_{N_{P}} \phi(g n) d n=0
$$

for every $g \in{ }^{\prime} G$ and every minimal parabolic subgroup ' $P$. Thus, if ' $G$ is a reductive Lie group of the Harish-Chandra class of split rank 1, then our definition of cusp forms coincides with the definition of Harish-Chandra; see Var77, Part II, Sect. 12.6, p. 222].

We now move on to investigate the relation between $\mathscr{C}_{\text {cusp }}(G / H)$ and $\mathscr{C}_{\text {ds }}(G / H)$. Our main tool will be the identity (8.9). The following result is a straightforward consequence of Theorem 3.6 . 
Corollary 8.18. $\mathscr{C}_{\mathrm{ds}}(G / H) \cap L^{1}(G / H) \subseteq \mathscr{C}_{\text {cusp }}(G / H)$.

Lemma 8.19. Let $Q \in \mathscr{P}_{\mathfrak{h}}(A)$ and $\phi \in \mathscr{C}(G / H: \tau)$. Then $\mathcal{H}_{Q, \tau} \phi=0$ if and only if both (a) and (b) hold:

(a) $\mathcal{I}_{Q, \tau} \phi=0$

(b) $\phi \perp \operatorname{Res}_{\tau}(Q, \mu) \psi$ for every $\mu \in S_{Q, \tau}$ and $\psi \in \mathscr{A}_{M, 2}(\tau)$.

In particular, if $\mathcal{H}_{Q, \tau} \phi=0$, then $\phi \in \mathscr{C}_{\mathrm{ds}}(G / H: \tau)$.

Proof. Assume $\mathcal{H}_{Q, \tau} \phi=0$. From [8.9) and Theorem 8.10 it follows for every $\psi \in \mathscr{A}_{M, 2}(\tau)$ that the function $\left\langle\mathcal{H}_{Q, \tau} \phi(\cdot), \psi\right\rangle$ equals a sum of the tempered term $\left\langle\mathcal{I}_{Q, \tau} \phi(\cdot), \psi\right\rangle$ and finitely many exponential terms with non-zero real exponents. Since $\mathcal{H}_{Q, \tau} \phi=0$, it follows that all the mentioned terms vanish. This proves (a) and (b) in the lemma. The converse implication follows directly from (8.9).

Finally, if (b) holds, then we infer from Corollary 8.2 that $\phi \in \mathscr{C}_{\mathrm{ds}}(G / H: \tau)$. This concludes the proof of the lemma.

Theorem 8.20. $\quad \mathscr{C}_{\text {cusp }}(G / H) \subseteq \mathscr{C}_{\mathrm{ds}}(G / H)$.

Proof. In view of Lemma 8.13 it suffices to show that every $K$-finite cusp form is an element of $\mathscr{C}_{\mathrm{ds}}(G / H)$. Let $\vartheta \subset \widehat{K}$ be finite and let $\tau$ and $\varsigma$ be as in Remark 8.16. Let $\phi \in \mathscr{C}_{\text {cusp }}(G / H)_{\vartheta}$ and assume that $Q \in \mathscr{P}_{\mathfrak{h}}(A)$. Then $\mathcal{H}_{Q, \tau}(\varsigma \phi)=0$ by Remark 8.16 and thus it follows from Lemma 8.19 that $\varsigma \phi \in \mathscr{C}_{\mathrm{ds}}(G / H: \tau)$. Hence, $\phi \in \mathscr{C}_{\mathrm{ds}}(G / H)_{\vartheta}$.

Remark 8.21. There exist symmetric spaces for which the inclusion of Theorem 8.20 is proper. Indeed, in AFJS12, Thm. 5.3] it has been established that the mentioned inclusion is proper for $G=\mathrm{SO}(p, q+1)_{e}$ and $H=\mathrm{SO}(p, q)_{e}$, with $1 \leq p<q-1$.

Theorem 8.22. If $\mathscr{C}_{\mathrm{ds}}(G / H)^{K} \subseteq \mathscr{C}_{\text {cusp }}(G / H)$, then $\mathscr{C}_{\mathrm{ds}}(G / H)=\mathscr{C}_{\text {cusp }}(G / H)$.

Proof. Assume $\mathscr{C}_{\mathrm{ds}}(G / H)^{K} \subseteq \mathscr{C}_{\text {cusp }}(G / H)$ and let $Q \in \mathscr{P}_{\mathfrak{h}}(A)$. Let $\mathscr{V}$ be the subspace of $C^{\infty}(G / H)^{K}$ spanned by the functions $\operatorname{Res}_{1}(Q: \mu) \psi$, for $\mu \in S_{Q, 1}$ and $\psi \in \mathscr{A}_{M, 2}(1)$. Then $\mathscr{V} \subseteq \mathscr{C}_{\mathrm{ds}}(G / H)^{K}$ by Proposition 17.18 , We claim that $\mathscr{V}=0$. To see this, let $\chi \in \mathscr{V}$. Then by the assumption and Remark 8.16 it follows that

$$
\mathcal{H}_{Q, 1}(\chi)=0 \text {. }
$$

By Lemma 8.19 (b) it follows that $\chi \perp \mathscr{V}$. Hence, $\chi=0$ and the claim is established.

We conclude from the claim and (8.9) that $\mathcal{H}_{Q, 1}=\mathcal{I}_{Q, 1}$. Let $\phi \in \mathscr{C}(G / H)$. By Proposition 4.2 there exists a $\widehat{\phi} \in \mathscr{C}(G / H)^{K}$ such that $|\phi| \leq \widehat{\phi}$. Now

$$
\left|\mathcal{H}_{Q} \phi\right| \leq \mathcal{H}_{Q}|\phi| \leq \mathcal{H}_{Q} \widehat{\phi} .
$$

Let $\psi_{0}$ be the element of $\mathscr{A}_{M, 2}(1) \simeq \mathbb{C}^{\mathscr{W}}$ determined by $\left(\psi_{0}\right)_{w}=\delta_{1, w}$. Then

$$
\mathcal{H}_{Q} \widehat{\phi}(a)=\left\langle\mathcal{H}_{Q, 1} \widehat{\phi}(a), \psi_{0}\right\rangle
$$

by (6.2); see Corollary 7.25, It follows from Proposition 7.2 that $\left\langle\mathcal{H}_{Q, 1} \widehat{\phi}(\cdot), \psi_{0}\right\rangle=$ $\left\langle\mathcal{I}_{Q, 1} \widehat{\phi}(\cdot), \psi_{0}\right\rangle$ is a tempered function on $A_{\mathfrak{q}}$. In view of the estimate (8.10) we conclude that $\mathcal{H}_{Q}$ maps the functions from $\mathscr{C}(G / H)$ to tempered functions on $L / H_{L}$. The same holds for $\mathcal{H}_{Q^{v}}$ with $v \in \mathscr{W}$.

Let $\left(\tau, V_{\tau}\right)$ be a finite dimensional representation of $K$. From (6.2) and Corollary 7.25 we conclude that $\mathcal{H}_{Q, \tau}$ maps the functions from $\mathscr{C}(G / H: \tau)$ to tempered 
$\mathscr{A}_{M, 2}(\tau)$-valued functions on $A_{\mathfrak{q}}$. It follows that the exponential terms in (8.9) are all equal to zero. Therefore, $\mathcal{H}_{Q, \tau}=\mathcal{I}_{Q, \tau}$ and by Corollary 8.2 we have that

$$
\mathscr{C}_{\mathrm{ds}}(G / H: \tau)=\operatorname{ker} \mathcal{H}_{Q, \tau} .
$$

Since this holds for every finite dimensional representation $\tau$ of $K$, we conclude that $\mathscr{C}_{\mathrm{ds}}(G / H) \subseteq \operatorname{ker}\left(\mathcal{R}_{Q}\right)$. As $Q \in \mathscr{P}_{\mathfrak{h}}\left(A_{\mathfrak{q}}\right)$ was arbitrary, we conclude that $\mathscr{C}_{\mathrm{ds}}(G / H) \subseteq \mathscr{C}_{\text {cusp }}(G / H)$. The converse inclusion was established in Theorem 8.20,

Remark 8.23. At present, we know of no example of a split rank one symmetric space where the equality $\mathscr{C}(G / H)^{K} \cap \mathscr{C}_{\text {cusp }}(G / H)=\{0\}$ is violated. On the other hand, $\mathscr{C}_{\text {ds }}(G / H) \cap \mathscr{C}_{\text {cusp }}(G / H)^{\perp}$ may contain irreducible submodules that are not spherical. An example of a symmetric pair for which this happens is provided by $G=\mathrm{SO}(p, q+1)_{e}$ and $H=\mathrm{SO}(p, q)_{e}$, with $1 \leq p<q-3$; see [AFJS12, Thm. 5.3].

Let $\left(\tau, V_{\tau}\right)$ be a finite dimensional unitary representation of $K$. Then we define $\mathscr{C}_{\text {cusp }}(G / H: \tau)$ to be the intersection of $\mathscr{C}(G / H: \tau)$ with $\mathscr{C}_{\text {cusp }}(G / H) \otimes V_{\tau}$. Furthermore, we define $\mathscr{C}_{\text {res }}(G / H: \tau)$ to be the $L^{2}$-orthocomplement of $\mathscr{C}_{\text {cusp }}(G / H: \tau)$ in $\mathscr{C}_{\mathrm{ds}}(G / H: \tau)$. Then by finite dimensionality of the latter space, we have the following direct sum decomposition:

$$
\mathscr{C}_{\mathrm{ds}}(G / H: \tau)=\mathscr{C}_{\mathrm{res}}(G / H: \tau) \oplus \mathscr{C}_{\text {cusp }}(G / H: \tau) .
$$

Theorem 8.24. Let $\mathscr{P}_{\mathfrak{h h}}(A)$ denote the set of $\mathfrak{h}$-extreme parabolic subgroups in $\mathscr{P}_{\mathfrak{h}}(A)$. Then

(a) $\mathscr{C}_{\text {cusp }}(G / H: \tau)=\left\{\phi \in \mathscr{C}(G / H: \tau): \mathcal{H}_{Q, \tau} \phi=0\left(\forall Q \in \mathscr{P}_{\mathfrak{h h}}(A)\right)\right\}$;

(b) $\mathscr{C}_{\mathrm{res}}(G / H: \tau)$ equals the space

$$
\operatorname{span}\left\{\operatorname{Res}_{\tau}(Q: \mu) \psi: Q \in \mathscr{P}_{\mathfrak{h h}}(A), \mu \in S_{Q, \tau}, \psi \in \mathscr{A}_{M, 2}(\tau)\right\} .
$$

Proof. If $\phi \in \mathscr{C}_{\text {cusp }}(G / H: \tau)$ and $Q \in \mathscr{P}_{\mathfrak{h h}}(A)$, then in view of (6.1) (see Corollary 7.25), it follows that $\mathcal{H}_{Q, \tau} \phi=0$. This establishes one inclusion. For the converse inclusion, assume that $\phi \in \mathscr{C}(G / H: \tau)$ belongs to the set on the right-hand side. Let $Q \in \mathscr{P}_{\mathfrak{h h}}(A)$. Then it follows from (6.1) (see Corollary 7.25), that $\left(\mathcal{R}_{Q} \otimes I_{V_{\tau}}\right)(\phi)$ vanishes on $L N_{Q}$. By sphericality of $\phi$ and $G$-equivariance of $\mathcal{R}_{Q}$ it follows that $\left(\mathcal{R}_{Q} \otimes I_{V_{\tau}}\right)(\phi)=0$. By Lemma 8.14 we infer that $\phi \in \mathscr{C}_{\text {cusp }}(G / H: \tau)$.

We now turn to (b). Let $\phi \in \mathscr{C}_{\mathrm{ds}}(G / H: \tau)$ and let $Q \in \mathscr{P}_{\mathfrak{h h}}(A)$. As $\mathcal{I}_{Q, \tau}$ vanishes on $\mathscr{C}_{\mathrm{ds}}(G / H: \tau)$ by Corollary 8.2 it follows from Lemma 8.19 that $\mathcal{H}_{Q, \tau} \phi=0$ if and only if $\phi$ is perpendicular to $\operatorname{Res}_{\tau}(Q, \mu) \psi$ for every $\mu \in S_{Q, \tau}$ and $\psi \in \mathscr{A}_{M, 2}(\tau)$. Therefore the space on the right-hand side of (b) equals the orthocomplement in $\mathscr{C}_{\mathrm{ds}}(G / H: \tau)$ of the space on the right-hand side of (a). Now (b) follows from (a) by the orthogonality of the direct sum (8.11).

Remark 8.25. In AFJS12, Thms. $5.2 \& 5.3$ ] it is shown that for the real hyperbolic spaces $\mathrm{SO}(p, q+1)_{e} / \mathrm{SO}(p, q)_{e}$, the left regular representation on $L_{\mathrm{ds}}^{2}(G / H) \cap$ $\mathscr{C}_{\text {cusp }}(G / H)^{\perp}$ is a finite direct sum of discrete series for $G / H$ and these are explicitly identified.

We conclude this article by giving a characterization of $K$-finite functions in $\mathscr{C}_{\text {ds }}(G / H)$.

Theorem 8.26. Let $\phi$ be a $K$-finite function in $\mathscr{C}(G / H)$. Then the following assertions are equivalent. 
(a) $\phi \in \mathscr{C}_{\mathrm{ds}}(G / H)$.

(b) For every $Q \in \mathscr{P}_{\mathfrak{h}}(A)$ and every $g \in G$ the function

$$
A_{\mathfrak{q}} \ni a \mapsto \mathcal{R}_{Q} \phi(g a)
$$

is a finite linear combination of real exponential functions.

(c) There exists an $\mathfrak{h}$-extreme $Q \in \mathscr{P}_{\mathfrak{h}}(A)$ such that for every $v \in \mathscr{W}$ and every $k \in K$ the function

$$
A_{\mathfrak{q}} \ni a \mapsto \mathcal{R}_{Q^{v}} \phi(k a)
$$

is a finite linear combination of real exponential functions.

Proof. Let $\vartheta$ be a finite subset of $\widehat{K}$ such that $\phi \in \mathscr{C}(G / H)_{\vartheta}$ and let $\tau$ and $\varsigma$ be as in Remark 8.16.

Assume that (a) is valid and let $Q \in \mathscr{P}_{\mathfrak{h}}(A)$. Then $\mathcal{I}_{Q, \tau}(\varsigma \phi)=0$ by Theorem 8.1. Therefore, only the exponential terms on the right-hand side of (8.9) can be non-zero. From the relation between $\mathcal{H}_{Q, \tau}$ and $\mathcal{H}_{Q}$ as given in (6.2) (see Corollary 7.25), it follows that the function (8.12) is of real exponential type, i.e., a finite linear combination of exponential functions with real exponents, if $g=e$. For $g=k \in K$ the assertion now follows from the $K$-equivariance of $\mathcal{R}_{Q}$. Let $g \in G$ be general, then $g=k a_{0} n_{Q}$ according to the Iwasawa decomposition $G=K A N_{Q}$. Furthermore,

$$
\mathcal{R}_{Q} \phi(g a)=\mathcal{R}_{Q} \phi\left(k a_{0} a\left(a^{-1} n_{Q} a\right)\right)=\mathcal{R}_{Q} \phi\left(k\left(a_{0} a\right)\right)
$$

and we see that (8.12) is of real exponential type. Hence, (b) follows.

Clearly, (b) implies (c). Now assume (c) and let $Q$ be an h-extreme parabolic subgroup in $\mathscr{P}_{\mathfrak{h}}(A)$ with the asserted properties. It follows from (6.2) (see Corollary 7.25), that for every $\psi \in \mathscr{A}_{M, 2}(\tau)$ the function $\left\langle\mathcal{H}_{Q, \tau}(\varsigma \phi)(\cdot), \psi\right\rangle$ is of real exponential type. From (8.9) we then read off that $\left\langle\mathcal{I}_{Q, \tau}(\varsigma \phi)(\cdot), \psi\right\rangle$ is of such exponential type as well. It now suffices to prove the claim that $\mathcal{I}_{Q, \tau}(\varsigma \phi)$ is in fact equal to 0 . Indeed, from the claim it follows that $\phi \in \mathscr{C}_{\mathrm{ds}}(G / H)_{\vartheta}$ by Corollary 8.2. Hence (a).

It remains to prove the above claim. We established for every $\psi \in \mathscr{A}_{M, 2}(\tau)$ that the function $\left\langle\mathcal{I}_{Q, \tau}(\varsigma \phi)(\cdot), \psi\right\rangle$ is of real exponential type. Since this function is tempered in view of Proposition [7.2 it has to be constant. As this is valid for every $\psi \in \mathscr{A}_{M, 2}(\tau)$, the support of $\mathcal{F}_{A_{\mathfrak{q}}}\left(\mathcal{I}_{Q, \tau}(\varsigma \phi)\right)$ is contained in the origin. Now it follows from (7.7) that $\mathcal{F}_{A_{\mathfrak{q}}}\left(\mathcal{K}_{Q, \tau}(\varsigma \phi)\right)$ is supported in the origin as well. As the latter is a smooth function, it must vanish identically. It then follows from (7.7) that also $\mathcal{I}_{Q, \tau}(\varsigma \phi)=0$. The validity of the claim follows.

\section{ACKNOWLEDGMENTS}

The authors are very grateful to Mogens Flensted-Jensen and Henrik Schlichtkrull for generously sharing their ideas in many enlightening discussions.

\section{REFERENCES}

[AFJS12] N. B. Andersen, M. Flensted-Jensen, and H. Schlichtkrull, Cuspidal discrete series for semisimple symmetric spaces, J. Funct. Anal. 263 (2012), no. 8, 2384-2408, DOI 10.1016/j.jfa.2012.07.009. MR2964687

[BvdB14] D. Bălibanu and E. P. van den Ban, Convexity theorems for semisimple symmetric spaces, Forum Math. 28 (2016), no. 6, 1167-1204, DOI 10.1515/forum-2015-0079. MR.3567864

[vdB86] E. P. van den Ban, A convexity theorem for semisimple symmetric spaces, Pacific J. Math. 124 (1986), no. 1, 21-55. MR850665 
[vdB87a] E. P. van den Ban, Asymptotic behaviour of matrix coefficients related to reductive symmetric spaces, Nederl. Akad. Wetensch. Indag. Math. 49 (1987), no. 3, 225-249. MR914083

[vdB87b] E. P. van den Ban, Invariant differential operators on a semisimple symmetric space and finite multiplicities in a Plancherel formula, Ark. Mat. 25 (1987), no. 2, 175-187, DOI 10.1007/BF02384442. MR.923405

[vdB88] E. P. van den Ban, The principal series for a reductive symmetric space. I. H-fixed distribution vectors, Ann. Sci. École Norm. Sup. (4) 21 (1988), no. 3, 359-412. MR.974410

[vdB92] E. P. van den Ban, The principal series for a reductive symmetric space. II. Eisenstein integrals, J. Funct. Anal. 109 (1992), no. 2, 331-441, DOI 10.1016/00221236(92)90021-A. MR 1186325

[vdBK14] E. P. van den Ban and J. J. Kuit, Normalizations of Eisenstein integrals for reductive symmetric spaces, J. Funct. Anal. 272 (2017), no. 7, 2795-2864, DOI 10.1016/j.jfa.2017.01.004. MR3608654

[vdBS97a] E. P. van den Ban and H. Schlichtkrull, Expansions for Eisenstein integrals on semisimple symmetric spaces, Ark. Mat. 35 (1997), no. 1, 59-86, DOI 10.1007/BF02559593. MR 1443036

[vdBS97b] E. van den Ban and H. Schlichtkrull, Fourier transform on a semisimple symmetric space, Invent. Math. 130 (1997), no. 3, 517-574, DOI 10.1007/s002220050193. MR.1483993

[vdBS97c] E. P. van den Ban and H. Schlichtkrull, The most continuous part of the Plancherel decomposition for a reductive symmetric space, Ann. of Math. (2) 145 (1997), no. 2, 267-364, DOI 10.2307/2951816. MR.1441878

[vdBS99] E. P. van den Ban and H. Schlichtkrull, Fourier inversion on a reductive symmetric space, Acta Math. 182 (1999), no. 1, 25-85, DOI 10.1007/BF02392823. MR.1687176

[vdBS00] E. P. van den Ban and H. Schlichtkrull, A residue calculus for root systems, Compositio Math. 123 (2000), no. 1, 27-72, DOI 10.1023/A:1002025119005. MR.1784755

[vdBS05] E. P. van den Ban and H. Schlichtkrull, The Plancherel decomposition for a reductive symmetric space. I. Spherical functions, Invent. Math. 161 (2005), no. 3, 453-566, DOI 10.1007/s00222-004-0431-y. MR2181715

[vdBS12] E. P. van den Ban and H. Schlichtkrull, Polynomial estimates for c-functions on reductive symmetric spaces, Int. Math. Res. Not. IMRN 6 (2012), 1201-1229, DOI 10.1093/imrn/rnr050. MR2899950

[Del98] P. Delorme, Formule de Plancherel pour les espaces symétriques réductifs (French), Ann. of Math. (2) 147 (1998), no. 2, 417-452, DOI 10.2307/121014. MR1626757

[DM78] J. Dixmier and P. Malliavin, Factorisations de fonctions et de vecteurs indéfiniment différentiables, Bull. Sci. Math. (2) 102 (1978), no. 4, 307-330. MR.517765

[HC66] Harish-Chandra, Discrete series for semisimple Lie groups. II. Explicit determination of the characters, Acta Math. 116 (1966), 1-111, DOI 10.1007/BF02392813. MR 0219666

[HC70] Harish-Chandra, Harmonic analysis on semisimple Lie groups, Bull. Amer. Math. Soc. 76 (1970), 529-551, DOI 10.1090/S0002-9904-1970-12442-9. MR0257282

[HC75] Harish-Chandra, Harmonic analysis on real reductive groups. I. The theory of the constant term, J. Functional Analysis 19 (1975), 104-204. MR0399356

[Hör03] L. Hörmander, The analysis of linear partial differential operators. I, 2nd ed., Grundlehren der Mathematischen Wissenschaften [Fundamental Principles of Mathematical Sciences], vol. 256, Springer-Verlag, Berlin, 1990. Distribution theory and Fourier analysis. MR.1065993

[KS80] A. W. Knapp and E. M. Stein, Intertwining operators for semisimple groups. II, Invent. Math. 60 (1980), no. 1, 9-84, DOI 10.1007/BF01389898. MR582703

[Kr09] B. Krëts, The horospherical transform on real symmetric spaces: kernel and cokernel, Funktsional. Anal. i Prilozhen. 43 (2009), no. 1, 37-54, DOI 10.1007/s10688-009-00043; English transl., Funct. Anal. Appl. 43 (2009), no. 1, 30-43. MR2503864

[KrS12] B. Krötz and H. Schlichtkrull. On function spaces on symmetric spaces. In Representation theory, complex analysis, and integral geometry, pages 1-8. Birkhäuser/Springer, New York, 2012.

[Kui13] J. J. Kuit, Radon transformation on reductive symmetric spaces: support theorems, Adv. Math. 240 (2013), 427-483, DOI 10.1016/j.aim.2013.03.010. MR3046316 
[Óla87] G. Ólafsson, Fourier and Poisson transformation associated to a semisimple symmetric space, Invent. Math. 90 (1987), no. 3, 605-629, DOI 10.1007/BF01389180. MR914851

[ŌM84] T. Oshima and T. Matsuki, A description of discrete series for semisimple symmetric spaces, Group representations and systems of differential equations (Tokyo, 1982), Adv. Stud. Pure Math., vol. 4, North-Holland, Amsterdam, 1984, pp. 331-390. MR810636

[Pou72] N. S. Poulsen, On $C^{\infty}$-vectors and intertwining bilinear forms for representations of Lie groups, J. Functional Analysis 9 (1972), 87-120. MR0310137

[Ros79] W. Rossmann, The structure of semisimple symmetric spaces, Canad. J. Math. 31 (1979), no. 1, 157-180, DOI 10.4153/CJM-1979-017-6. MR518716

[Var77] V.S. Varadarajan, Harmonic analysis on real reductive groups, Lecture Notes in Mathematics, Vol. 576, Springer-Verlag, Berlin-New York, 1977. MR0473111

[Wal17] N. R. Wallach. On the Whittaker Plancherel Theorem for Real Reductive Groups. arXiv:1705.0678\%.

[Wal88] N. R. Wallach, Real reductive groups. I, Pure and Applied Mathematics, vol. 132, Academic Press, Inc., Boston, MA, 1988. MR929683

[Wal92] N. R. Wallach, Real reductive groups. II, Pure and Applied Mathematics, vol. 132, Academic Press, Inc., Boston, MA, 1992. MR.1170566

Mathematical Institute, Utrecht University, PO Box 80 010, 3508 TA Utrecht, The NETHERLANDS

E-mail address: e.p.vandenban@uu.nl

Institut für Mathematik, Universität Paderborn, WARburger Strasse 100, 33089 PAderborn, Germany

E-mail address: j.j.kuit@gmail.com 